\title{
Removing Redundancy and Reducing Fitness Evaluation Costs in Genetic Programming
}

\author{
by \\ Phillip Lee-Ming Wong
}
A thesis
submitted to the Victoria University of Wellington in fulfilment of the requirements for the degree of Master of Science in Computer Science.

Victoria University of Wellington 2008 



\begin{abstract}
One of the greater issues in Genetic Programming (GP) is the computational effort required to run the evolution and discover a good solution. Phenomena such as program bloating (where genetic programs rapidly grow in size) can quickly exhaust available memory resources and slow down the evolutionary process, while the heavy cost of performing fitness evaluation can make problems which have a lot of available data very slow to solve. These issues may limit GP in some tasks it can appropriately be applied to, as well as inhibit its applications in time/space sensitive environments.

In this thesis, we look at developing solutions to some of these issues in GP computational cost. First, we develop an algebraic program simplification method based on simple rules and hashing techniques, and use this method in conjunction with the standard GP on a variety of tasks. Our results suggest that program simplification can lead to a significant reduction in program size, while not significantly changing the effectiveness of the systems in finding solution programs. Secondly, we analyse the effects of program simplification on the internal GP "building blocks" to investigate whether simplification is a destructive or constructive force. Using two models for building blocks (numerical-nodes and the more complex fixed-depth subtree), we track building blocks through GP runs on a symbolic regression problem, both with and without using simplification. We find that the program simplification process can both disrupt and construct building blocks in the GP populations. However, GP systems using simplification appear to retain important building blocks, and the simplification process appears to lead to an increase in genetic diversity. These
\end{abstract}


may help explain why using simplification does not reduce the effectiveness of GP systems in solving tasks.

Lastly, we develop two methods of reducing the cost of fitness evaluation by reducing the number of node evaluations performed. The first method is elitism avoidance, which avoids re-evaluating programs which have been placed in the population using elitism reproduction. This method reduces the CPU time for evolving solutions for six different GP tasks. The second method is a subtree caching mechanism which store fitness evaluations for subtrees in a cache so that they may be fetched when these subtrees are encountered in future fitness evaluations. Results suggest that using this mechanism can significantly reduce both the number of node evaluations and the overall CPU time used in evolving solutions, without reducing the fitness of the solutions produced. 


\section{Acknowledgments}

Firstly, a very special thanks to Dr. Mengjie Zhang for the amount of effort he put in supervising me, providing extremely helpful feedback for this thesis and with co-authoring several papers.

I would also like to thank my family, my sister Mei-Lin, my mother Lee-Lee and my father Frank for their constant support throughout my journey through academia.

Some other people I would like to thank (for various reasons, including keeping me sane): Melissa Gyetse, Matthew Lock, Isaac Sansom, Martin Chin.

Lastly, thanks to the VUW Genetic Programming Group, for providing both ideas and criticisms.

This work was supported in part by the Royal Society of New Zealand Marsden Fund. 


\section{Contents}

1 Introduction $\quad \mathbf{1}$

1.1 Genetic Programming . . . . . . . . . . . . . . . . 1

1.2 Issues in GP . . . . . . . . . . . . . . . . . 2

1.2.1 Program Bloat and Redundancy ......... . 2

1.2.2 Fitness Evaluation . . . . . . . . . . . 4

1.3 Thesis Goals . . . . . . . . . . . . . . . . . . . . . . 4

1.4 Major Contributions . . . . . . . . . . . . 6

1.5 Structure .................... 8

2 Literature Survey 9

2.1 Background Concepts . . . . . . . . . . . . . . 9

2.1 .1 Machine Learning . . . . . . . . . . . . 9

2.1.2 Evolutionary Computation . . . . . . . . . . . . . 12

2.1.3 Genetic Programming . . . . . . . . . . . . . . 12

2.2 Related Work to Program Bloating . . . . . . . . . . . . 18

2.2.1 The Problem, and Causes . . . . . . . . . 18

2.2.2 Basic Bloat Control: Depth and Size Limiting . . . . . 21

2.2.3 Parsimony Pressure . . . . . . . . . . . . . 21

2.2.4 Other Bloat Control Methods . . . . . . . . . . . . 23

2.2.5 Simplification of GP Programs . . . . . . . . . . . 24

2.3 Related Work to Building Blocks . . . . . . . . . . . . . 26

2.3.1 Building Block Hypothesis and Schema Theory in GA 27

2.3.2 Building Block Hypothesis and Schema Theory in GP 27 
2.3.3 Empirical Analysis . . . . . . . . . . . . . . . . 29

2.4 Related Work to Fitness Evaluation . . . . . . . . . . . . 30

2.5 Chapter Summary . . . . . . . . . . . . . . . . . . . . 34

3 Datasets 37

3.1 Symbolic Regression . . . . . . . . . . . . . . . 37

3.2 Image Classification . . . . . . . . . . . . . . . . 39

3.3 Medical Classification . . . . . . . . . . . . . . 43

3.4 GP Settings . . . . . . . . . . . . . . . . . . . . . . 45

3.4.1 Common Parameters . . . . . . . . . . . . . . 45

3.4.2 VGP and Machine Environment . . . . . . . . 46

4 Algebraic Simplification of GP Programs 49

4.1 Introduction . . . . . . . . . . . . . . . . . . 49

4.1 .1 Chapter Goals . . . . . . . . . . . . . . 50

4.2 Algebraic Simplification . . . . . . . . . . . . . . 51

4.2.1 The Simplification Rules . . . . . . . . . . . . . . 51

4.2.2 The Simplification Process . . . . . . . . . . . . . 52

4.2.3 Estimating Algebraic Equivalence using Hashing . . 54

4.2.4 An Example . . . . . . . . . . . . . . . 61

4.2.5 Summary of the Simplification Algorithm . . . . . . . 64

4.3 Experimental Setup . . . . . . . . . . . . . . . . . 65

4.4 Results and Discussion . . . . . . . . . . . . . . . 65

4.5 Chapter Summary . . . . . . . . . . . . . . . . . 72

5 Effects of Simplification on Simple Building-Blocks 75

5.1 Introduction . . . . . . . . . . . . . . . . . 75

5.1 .1 Chapter Goals . . . . . . . . . . . . . 76

5.2 Numerical-Nodes as Simple Building Blocks . . . . . . . . 76

5.2.1 Visualisation and Interpretation of Numerical-Nodes 78

5.2.2 Experimental Setup . . . . . . . . . . . . . 79

5.3 Results and Discussion . . . . . . . . . . . . . . . 81 
5.3.1 Analysis: Disruption of Existing Building Blocks . . . 82

5.3.2 Analysis: Construction of New Building Blocks . . . 84

5.3.3 Effect of Construction/Disruption on Performance . 85

5.4 Chapter Summary . . . . . . . . . . . . . . . . . . 89

6 Fixed-Depth Subtree Building Blocks 91

6.1 Introduction . . . . . . . . . . . . . . . . 91

6.1 .1 Chapter Goals . . . . . . . . . . . . . . . . . . 91

6.2 Fixed-Depth Program Subtrees . . . . . . . . . . . . . . 92

6.2 .1 Identifying Subtrees . . . . . . . . . . . . . 93

6.3 Visualising Fixed Size Subtrees in GP . . . . . . . . . . . . 95

6.3.1 Using a Global Indexing Table . . . . . . . . . . 96

6.3.2 Using a Hashing Function . . . . . . . . . . . . . 98

6.4 FST-Hash: Hashing for Fixed-Depth Subtrees . . . . . . . . . 99

6.4.1 Producing a Level Ordering . . . . . . . . . . . . . 100

6.4.2 Translating the Ordering to a Hash . . . . . . . . . . 102

6.4.3 Plotting Hash Values . . . . . . . . . . . . . . . 103

6.5 Experimental Setup . . . . . . . . . . . . . . . . . . . 107

6.6 Results and Discussion . . . . . . . . . . . . . . . . . 107

6.6.1 Analysing Individual Runs . . . . . . . . . . . . . 108

6.6.2 Totaling Disrupted and Created Subtrees . . . . . . . 122

6.7 Chapter Summary . . . . . . . . . . . . . . . . . . . . 127

7 Reducing Costs of Fitness Evaluations 129

7.1 Introduction . . . . . . . . . . . . . . . . . . . . . . . . 129

7.1.1 Chapter Goals . . . . . . . . . . . . . . . . . 130

7.2 Avoiding Re-evaluation of Reproduced Programs . . . . . . 131

7.2.1 Experiments . . . . . . . . . . . . . 132

7.3 Avoiding Re-evaluation by using Subtree Caching . . . . . . 135

7.3.1 Calculating the number of possible distinct trees of depth $n \ldots \ldots \ldots \ldots$. . . . . . . . . . . . . . . . . . . . 
7.3.2 Subtree Caching using a Hashing for Equivalence MEthod (SCHEME) . . . . . . . . . . . . . . 139

7.3.3 Experimental Setup . . . . . . . . . . . . . . 145

7.3.4 Results . . . . . . . . . . . . . . 146

7.4 Discussions of Caching Depth . . . . . . . . . . . . . 150

7.5 Discussions on Increasing the Number of Fitness Cases . . . 152

7.6 Selecting Caching Depth Dynamically . . . . . . . . . . . . . 157

7.6.1 dSCHEME: Caching Dynamically Chosen Subtrees . 157

7.6.2 iSCHEME: Caching using Incremental Depth . . . . . 159

7.7 Chapter Summary . . . . . . . . . . . . . . . . . . . . 161

8 Conclusions 165

8.1 Conclusions . . . . . . . . . . . . . . . . 165

8.1.1 Direct Conclusions . . . . . . . . . . . . 166

8.1 .2 Indirect Conclusions . . . . . . . . . . . . 170

8.2 Future Works . . . . . . . . . . . . . . . . . 171 


\section{Chapter 1}

\section{Introduction}

In this chapter we will introduce general concepts of GP, identify some current problems with GP today and form some research questions/goals which this thesis will aim to answer. We will also summarise the major contributions that we have made and the overall organisation of this thesis.

\subsection{Genetic Programming}

Genetic programming (GP) [40] is a method of automatically generating programs for solving specific tasks based on concepts loosely derived from Darwinian Evolution. Firstly, an initial group of randomly generated genetic programs, normally represented as parse trees such as LISP-S trees, is created. The process of selection based on fitness is carried out to provide a basis for the next program generation. Fitness is determined by running the programs and evaluating them on a set of criteria called fitness function. The genetic operators of crossover for swapping of sections of programs, mutation for random alterations to a program, and reproduction for retaining the best programs are applied to the selected programs to create a new population of programs. The process of creating new generations is repeated until certain termination criterion is met. The "best" program in 
the last generation is usually used as the resulting system solution. GP can be seen as a genetic beam search through the space of possible solutions to the task.

GP is an emerging field in evolutionary computing and machine learning and has already been applied to many tasks, including image analysis [66], object detection [100], regression problems [40] and even control programs for walking robots [11]. GP has been very successful in solving or performing these tasks and "now routinely delivers high-return humancompetitive machine intelligence" $[41,42]$.

\subsection{Issues in GP}

GP can be fairly computationally intensive when training programs, especially when trying to evolve solutions to more difficult tasks. Often these tasks will have more complex and intricate solutions which may require a larger program tree to encompass and larger GP populations to find. As such, GP may slow down significantly as evolution progresses or even halt completely. It is essential that we reduce the computational effort required by GP.

\subsubsection{Program Bloat and Redundancy}

Program bloating is a well-documented fundamental problem that affects GP today $[40,88,10]$. As the GP evolution progresses, there is a tendency for programs to grow rapidly, in reaction to the destructive nature of the GP crossover operator. This growth, which is largely made up of redundant code (functionally contributes nothing to the program's output), is detrimental to the GP process, capable of quickly exhausting a system's available memory and possibly prematurely halting the process before a good solution can be found. It also unnecessarily explores regions of the search space which are not beneficial, which may even lessen the effec- 
tiveness in finding a good solution. Redundant code can also result in an unnecessarily complex program, which is inefficient in its execution and difficult to interpret and comprehend.

There have been several approaches to controlling this bloating. These include methods of indirectly reducing redundancy (e.g. Parsimony Pressure [40, 99, 58, 87, 50], Edge Marking [10], Explicitly Defined Introns [59]), as well as methods which directly identify and remove the redundant code (e.g. Simplification $[19,101])$. Methods such as Parsimony Pressure have shown limitations (i.e. performance is task dependent [50], premature GP population convergence [58]), while previous simplification methods have required translation of GP programs into alternate representations (e.g. program string [19], prime number encoding [101]) before simplification can be applied. Ideally, we would like to have an approach to control redundancy growth which can be applied to a variety of tasks, and which requires as little manipulation of the GP programs as necessary.

On the other hand, this redundancy may aid the effectiveness of the evolutionary process by providing a more diverse selection of program fragments for the process to use, and protecting useful "building blocks" (building blocks are small pieces of code which contribute highly towards the overall fitness of the program) within programs from the destructive nature of the crossover operator [10]. In other words, they are very important and useful subparts of the programs. By removing these redundancies, we may be exposing these building blocks to the crossover operator, breaking them up and causing the fitness of the programs to fall. If this is so, the effectiveness of GP in finding a good solution may be hindered. It is necessary to investigate the relationship between the redundancy and the effectiveness of GP, as well as how removing this redundancy affects building blocks within the GP populations. 


\subsubsection{Fitness Evaluation}

Fitness evaluation is the most expensive procedure in GP [24]. In each generation, all programs typically need to be evaluated for fitness, which is then used by genetic selection. For many tasks, such as classification, this can mean evaluating a single program on hundreds (or thousands) of fitness cases in order to determine that program's classification accuracy. Taking a typical GP system with 500 programs per generation, 50 generations of evolution and 500 fitness cases, we arrive at 12,500, 000 fitness evaluations that need to be performed throughout the process. It is clear that reducing the cost of fitness evaluations is necessary to improving the efficiency and applicability of GP. Therefore it is necessary to find appropriate methods of reducing this cost.

\subsection{Thesis Goals}

The overall goal of this thesis is to improve the GP process to increase its efficiency, thereby improving GP's utilisation of available system resources. We can split this overall goal into two subgoals. The first subgoal is to combat program bloat and reduce the memory usage that GP requires during evolution. This is important in allowing GP to be applied to more difficult tasks, as well as allowing GP to be used in more constrained environments. The second subgoal is to decrease the amount of training time needed for GP to find a solution. This is also important for the applicability of GP in environments with strict time requirements (e.g. industry applications).

In order to achieve these goals, this thesis will focus on answering the following research questions.

\section{How can program bloat be reduced?}

The program bloating problem is a significant source of resource usage in GP and a key issue in GP. In this thesis, we will develop a pro- 
gram simplification method based on algebraic rules which directly removes superfluous code from programs during the evolutionary process. We expect that the use of this simplification method will be able to improve the evolutionary training efficiency while maintaining the effectiveness of the GP solutions.

2. Will removing the bloat worsen GP performance?

It has been theorised that program bloating is an automatic response to the destructive properties of the GP crossover operator. By having large amounts of "useless" code, the probability of crossover choosing a crossover point within another, more important section of code is low. By removing the program bloat, simplification may lead to a disruption of GP "building-blocks", which may be vital to the success of a GP run. Consequently, we may see a deterioration in the effectiveness of GP to solve the given task.

To test this hypothesis, we will examine "building-blocks" within the GP populations during GP evolution, both with and without simplification being used. This empirical analysis will give us a deeper understanding of the effects of simplification on GP.

3. How can fitness evaluation be made more efficient?

A majority of existing works for reducing fitness evaluation costs in GP have fallen into two approaches: refining the fitness cases or refining the fitness evaluation procedure. We aim to develop a new approach to reducing the number of node evaluations required to evaluate GP programs using caching methods, and hence make fitness evaluation more efficient. 


\subsection{Major Contributions}

This thesis makes the following major contributions towards the field of GP.

1. Algebraic Simplification. We develop an algebraic simplification algorithm which uses algebraic simplification rules and our own Algebraic Equivalence Hashing method to directly simplify tree-based programs within GP populations during the evolutionary process. This work shows how the redundant code in evolved genetic programs can be directly removed during the evolutionary process. Our results show that simplification is able to reduce the size of programs within GP, and when not performed at every generation, is able to reduce the CPU time required to evolve solutions. They also show that using simplification does not reduce the effectiveness of GP in finding good solutions.

Part of this work has been published in:

- Mengjie Zhang and Phillip Wong. "Genetic Programming for Medical Classification: A Program Simplification Approach". Genetic Programming and Evolvable Machines. Vol. 9, 2008. 27pp. DOI 10.1007/s10710-008-9059-9. (online)

- Phillip Wong, Mengjie Zhang. "Algebraic Simplification of GP Programs during Evolution". Proceeding of Genetic and Evolutionary Computation Conference 2006 (GECCO 2006). ACM Press. pp. 927-934.

2. Building Block Analysis of Simplification. We developed two building-block models (numerical-nodes, fixed-depth subtrees) as well as our own methodology for analysing and visualising these building-blocks as they interact in GP runs. Using these models/methodologies we provide an analysis of the effects of using our simplification method 
on the internal building-blocks of many GP runs on a symbolic regression problem. This analysis improves our understanding of how using program simplification affects GP systems beyond directly removing redundant code. Our results show that simplification both disrupts and constructs building blocks.

Parts of this work has been published in:

- Phillip Wong, Mengjie Zhang. "Numerical-Node Building Block Analysis of Genetic Programming with Simplification". Genetic and Evolutionary Computation Conference (GECCO'07). ACM Press. 2007.

- Phillip Wong, Mengjie Zhang. "Effects of Program Simplification on Simple Building Blocks in Genetic Programming". Proceedings of the 2007 IEEE Congress on Evolutionary Computation. IEEE Press. Singapore. 2007. pp. 1570-1577.

3. Subtree Caching in Fitness Evaluation. We developed a method of caching evaluations of program subtrees during GP evolution in order to avoid re-evaluating subtrees that re-occur in the GP populations during the evolutionary process. This work shows how we can reduce the number of node evaluations that the fitness evaluation procedure needs to perform, and therefore reduce its computational effort. Our results show that we can significantly reduce both the number of node evaluations performed, as well as the CPU time needed for GP to evolve solutions, without reducing the effectiveness of the programs produced.

Part of this work has been published in:

- Phillip Wong, Mengjie Zhang. "SCHEME: Caching Subtrees in Genetic Programming". Proceedings of the 2008 IEEE Congress on Evolutionary Computation. IEEE Press. Hong Kong. June 2008. pp. 2683-2690. 


\subsection{Structure}

The remainder of this thesis will be structured as follows. Chapter 2 provides a detailed discussion of background concepts and work related to this thesis. Chapter 3 describes the settings and parameters used for the GP systems in this thesis, as well as describing each of the datasets used for experiments.

Chapter 4 investigates our first research question and describes our simplification system for removing program bloat. We integrate this simplification system with the standard GP and perform experiments using several tasks.

Chapters 5 and 6 address our second research question. Chapter 5 presents our first approach (using numerical-nodes), including our methodology for analysing and investigating the effect of simplification on these numerical-nodes. In chapter 6, we expand on this simple building-block model and look at fixed-depth subtrees, again investigating the effect of simplification on these building-blocks.

Chapter 7 looks at our third and final research question. It presents our methods of avoiding fitness evaluation through exploiting elitism and caching fitness evaluations for subtrees.

Finally, chapter 8 summarises the major conclusions we found during the work for this thesis, and discusses possible future directions. 


\section{Chapter 2}

\section{Literature Survey}

\subsection{Background Concepts}

In this section we will explain some of the background concepts surrounding the work in this thesis. The descriptions will only present a brief overview of these concepts to familiarise the reader. However, citations are provided for further reading if more in depth information is desired.

\subsubsection{Machine Learning}

Machine Learning (ML) [56, 2, 51, 9] is a large sub-field of Artificial Intelligence that concerns developing techniques that allow computers to "learn". It is mainly focused on the automatic extraction of information from available data. Common applications for machine learning include: natural language processing, medical diagnosis, handwriting recognition, financial analysis, speech processing etc.

Machine learning methods themselves are split into a number of techniques. These include: supervised learning, unsupervised learning, reinforcement learning, transduction, semi-supervised learning etc. In this work, we are only concerned with supervised learning. 


\section{Supervised Learning}

Supervised Learning is one of the many learning techniques used in machine learning. It consists of using a set of labelled training instances from which the learning system derives a desired function (i.e. a classifier) that maps input data to desired output. The labels are provided by "experts" in the particular task the data is used for.

If all of the possible inputs and outputs are known then this task is relatively straightforward (and can be simply and efficiently accomplished using a look-up table). However, most problems for supervised learning contain intractable numbers of possible input combinations. Hence, the amount of data available to a learner is usually only a subset of the possible data. The aim of a good learner is to be able to correctly work on new data that it has not seen before (i.e. generalise to unseen data).

Because of this aim, a learning method often needs to be monitored or controlled to avoid overfitting [91, 1]. Over the course of learning, there will be a point where the learner can still perform increasingly well on the data it is trained on, but its performance on unseen test data drops. At this point, we say that the learner is overfitting the training data.

Overfitting can be seen as a model with an excess of parameters for a particular problem [51]. In figure 2.1, we have half a dozen data points which have an output $y$ given a particular value $x$. Also in the figure we have two models that fit this set of data. The first is a straight line passing through all the points, while the second is a complex curve which also passes through all the data points. Even though both of these models can correctly predict the outputs for the seen data, it is unlikely that the complex model will perform well on unseen data. In line with Occam's razor, it is often best (all else being equal) to choose the simpler model with fewer parameters that describes the data.

There are a multitude of ways to avoid overfitting, but the most common of these is perhaps early stopping. In addition to the training data and the testing data, we have a third, validation data set. The validation set is 


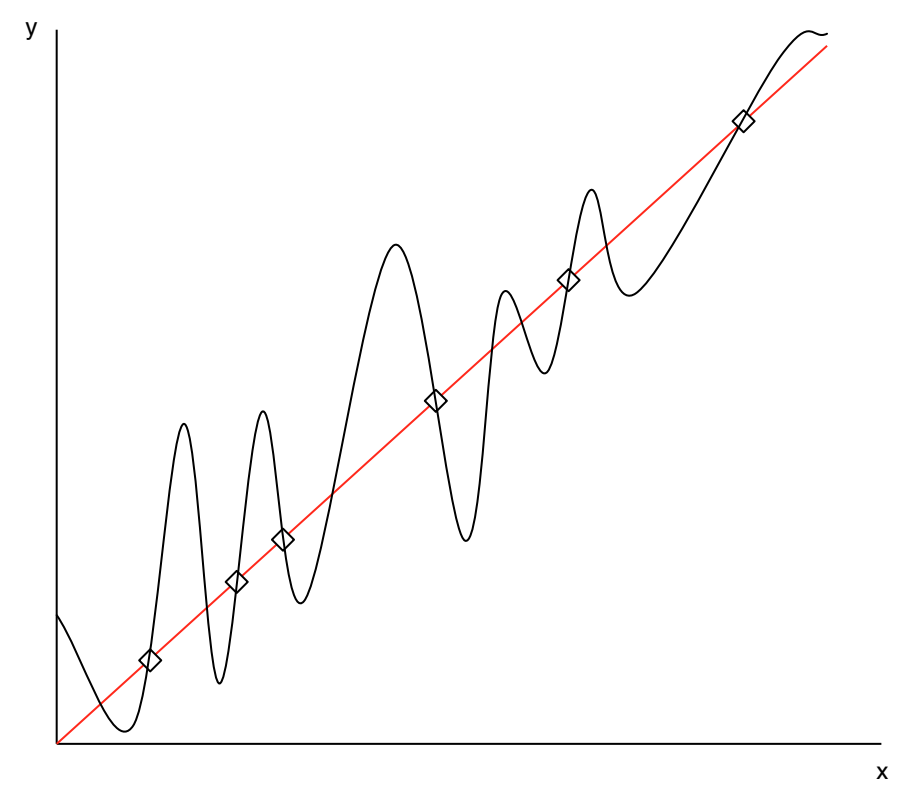

Figure 2.1: Simple example of an overfitting model

used to monitor the training process. While the learner does not directly learn from the validation set data, it is periodically tested on the validation data to see if overfitting has occurred (estimated through observing repeated drops in performance on validation data).

In some cases, the amount of available data is very small, making it difficult to split the data into sufficiently sized training, testing and validation sets. In these cases, we use a technique called $k$-fold cross-validation [39] (also sometimes called rotation estimation). This is performed by dividing the available data into $k$ number of equally sized sets or folds ( $k$ is user-defined, i.e. $k=10,10$-fold cross-validation). The learning method is trained and tested $k$ number of times, each time using a different fold for testing and the remaining $k-1$ folds for training the system. An average of the results for the $k$ folds can be used as a single estimation for the system 
being tested.

\subsubsection{Evolutionary Computation}

Evolutionary Computation (EC) [30, 18] is another sub-field of Artificial Intelligence which involves developing solutions to problems, using evolutionary techniques often inspired by biological concepts in evolution. EC methods maintain a collection or population of structures (e.g. programs, binary strings, etc.) on which genetic operators are applied. These operators often borrow from biological evolution, resulting in operators such as reproduction, mutation, and selection.

The process of selection is based on the desire to retain highly fit individuals, while ignoring unfit individuals. Selected programs are then used for other genetic operators such as mutation or crossover. By using selection, programs in the next generation are likely to be derived from more fit individuals in the current generation. Selection requires that all individuals in a population be evaluated for fitness (i.e. how well the individual is performing), an often costly procedure.

There are many paradigms (algorithms) in EC, including (but not limited to) Genetic Algorithms [55], Genetic Programming, Evolution Strategies [8], Evolutionary Programming [18], Particle Swarm Optimization [38] and Ant Colony Optimization [17]. As this thesis is concerned with Genetic Programming, we will give a brief review of GP in the next subsection.

\subsubsection{Genetic Programming}

Genetic Programming (GP) $[72,47]$ is an EC method popularised by Koza in 1992 [40]. GP is a form of evolutionary computation, in which the structures being evolved are computer programs. These programs are often LISP-S type expressions stored in a tree-structure, but can also be other program structures, including graph [65], linear [57, 20, 7, 62] or gram- 
matical $[93,96]$ structures. Poli et al. [72] provides an excellent primer to GP and is a highly recommended read. As this thesis is focused on tree based GP (TGP), we will briefly review TGP in the rest of this subsection.

For standard tree-based GP, the values are taken from the terminal nodes and fed through the connected function nodes up to the root of the tree producing a single numeric value as the output.

\section{Terminal and Function Set}

The nodes of a GP program are selected from the terminal and function sets, which can be designed for the particular task being worked on. The terminal set often consists of a number of feature terminals which correspond to various features in the task. The terminal set usually also includes a number of randomly valued numeric terminals (also called ephemeral random constants [ERCs] [40]) which allows the GP system to construct numeric constants which may be required for making a solution to the given task.

The function set provides functions which are used to manipulate the terminal node values and build up the output for the GP program. The arithmetic operators $(+,-, \times, \div)$ combined with an if operator are the most commonly used functions in GP. In our work we use the if $<0$ variant of the if operator. The if $<0$ operator takes three arguments. The first is used as the condition and is evaluated to determine if the result is less than zero. If the condition passes (is less than zero), then the result of the second argument is passed as the result of the if $<0$ operator. If the condition fails, then the third argument is passed up instead. Other mathematical functions such as absolute, trigonometric functions (sine, cosine, tangent) and logarithmic functions are also fairly common to use in the function set, depending on the amount of prior knowledge known about the nature of the problem.

There are two additional requirements that the terminal and functions sets need to satisfy. The first is that the terminal and function sets must 
have closure. Closure requires that any function in the function set must be able to take any value from a terminal or function as an input. Because of this, it is common practice to use a special form of division:protected divison. In protected division, a division by zero is defined to return the value zero, removing the undefined case. The second requirement is sufficiency. That is, that the contents of the terminal and function sets should be enough to solve the given problem. However, prior knowledge about certain problems can often be limited, and it can be difficult to determine beforehand if a terminal and function set are sufficient for those problems.

\section{Population Initialisation}

As in most other evolutionary computation techniques, the initial population for GP is randomly generated. The two earliest and most common approaches to creating these random trees are the grow and full methods.

In the full method, trees are filled to their maximum size (where all leaf nodes are at the same depth). This is accomplished by selecting only function nodes for each branch until the maximum tree depth is reached, whereupon only terminal nodes can be selected to complete the tree. All program trees produced using this initialisation method are similar in shape, since all branches are completely filled (though different operators may have differing numbers of children).

In the grow method, the tree nodes are chosen from the combined set of terminals and functions until the maximum depth is reached on any of its branches. At this point, only terminals can be chosen to complete the tree. Using this method results in a bigger variety of tree shapes.

A lot of people use a combination of these two methods when creating the initial population, creating half of the population using one method and the other half using the other. This combination of methods is called ramped half-and-half. This method uses a range of depth limits in order to ensure that the initial population has a wide variety of tree sizes and shapes. 


\section{Selection}

There are two selection methods which are most commonly used in genetic programming. The first of these is fitness-proportionate selection. In fitness-proportionate selection, each program in the GP population is allocated a chance of being selected, where the chances of a particular program being selected is proportional to that program's fitness. In this way, more fit programs are more likely to be selected, while not entirely excluding less fit programs.

The other popular method is tournament selection. In this method, a number of programs are randomly chosen from the population and placed into the tournament. Then these programs are compared with each other and the fittest program is selected as the winner. Tournament selection has been shown to balance out selection pressure [47] so that a single highly fit program cannot overpower the next generation.

\section{Crossover}

The most common crossover (subtree crossover) operator takes two GP programs and selects a crossover point within each of those program. The subtrees of both programs originating at their respective crossover points are then swapped, resulting in two new GP programs. Both of these newly created GP programs are then inserted into the new GP population. An example of this type of crossover is shown in figure 2.2.

\section{Mutation}

The most common mutation operator used in GP selects a mutation point within a selected program. The subtree rooted at that point is then replaced with a new randomly generated subtree, although it is sometimes implemented by crossing over a program with an entirely new randomly generated program. An example of this type of mutation is shown in figure 2.3. 


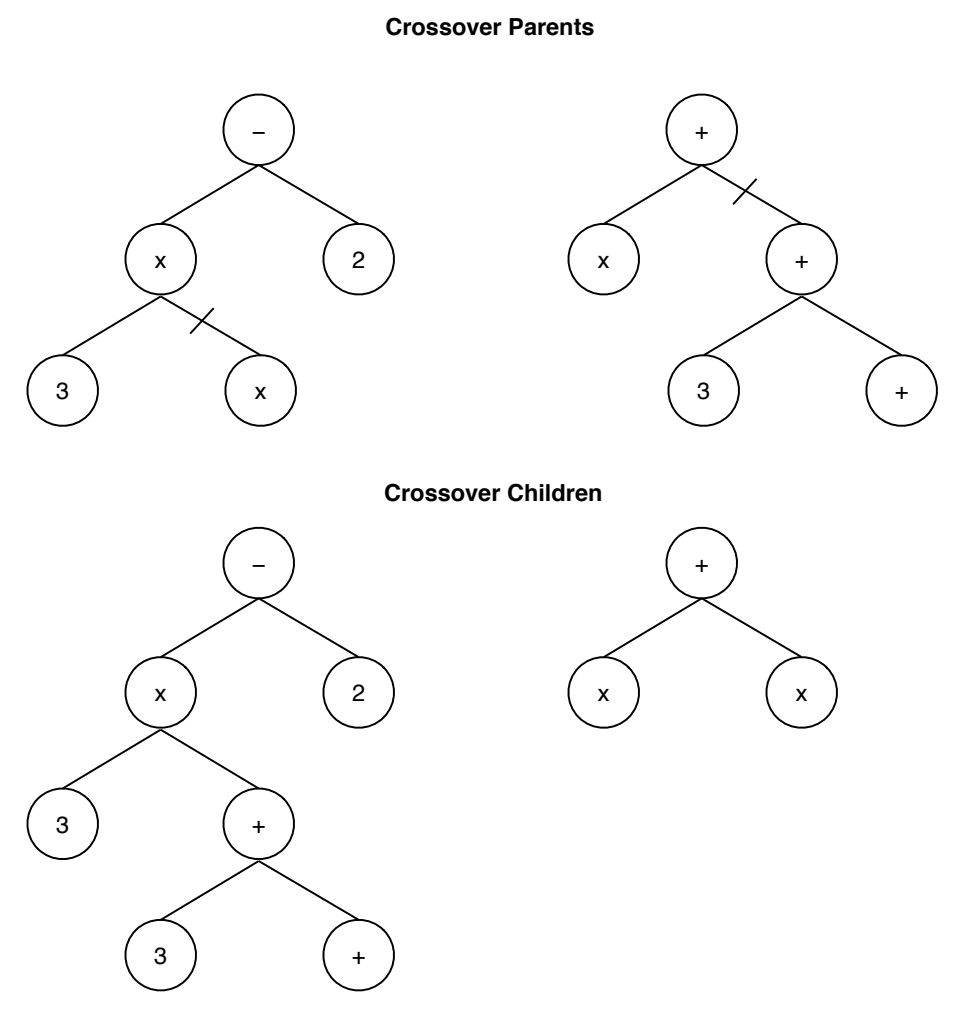

Figure 2.2: Simple example of GP crossover

\section{Reproduction}

Reproduction is a simple genetic operator where a selected program is simply copied, without modification, to the new generation. A common method of reproduction is elitism. In elitism, the normal selection method is not used, instead the programs in the population are ranked according to their fitness and a number of the highest ranked individuals are copied directly into the new population. By using elitism, it is ensured that the best program in the next generation is at least as good as the best program in the current generation. 

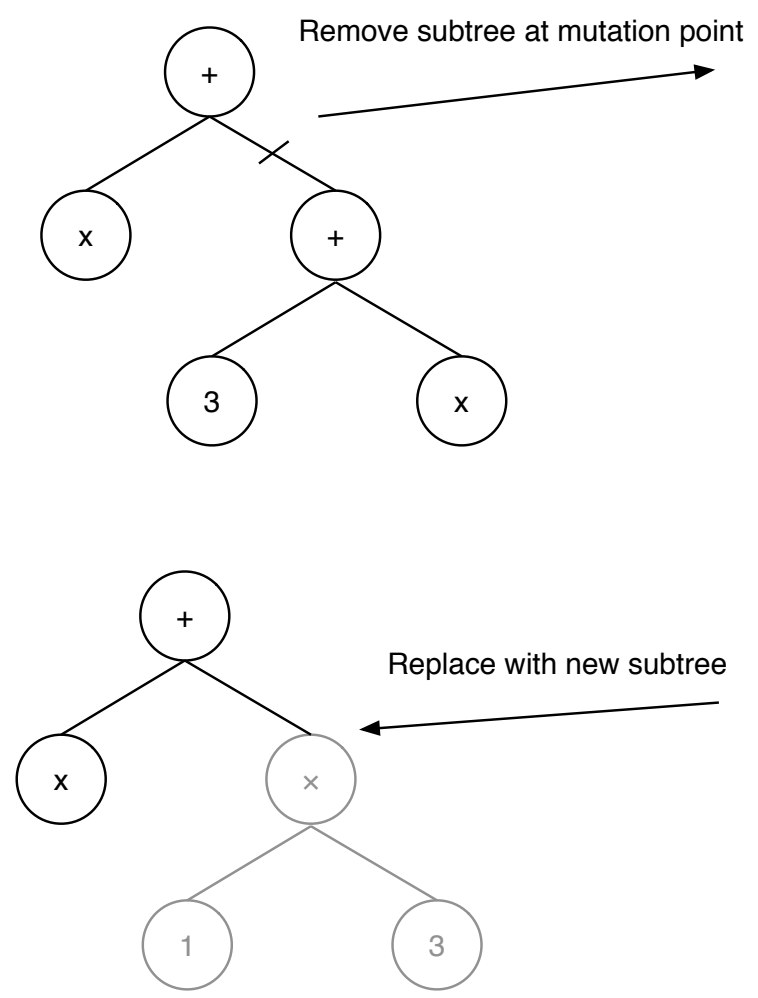

Figure 2.3: Simple example of GP mutation 


\subsection{Related Work to Program Bloating}

\subsubsection{The Problem, and Causes}

The problem of program bloat is a well documented problem which has been known since the beginning of GP. Koza describes this phenomenon in [40], where GP programs tend to grow excessively large. This subsection describes some typical work that has been performed on this phenomenon.

The main issue with this growth is the rate at which it occurs. Langdon claims in [43] that the average growth in program depth is linear when using the standard genetic operators (approximately one level per generation). As each additional depth contains increasingly more nodes, this leads to a trend in GP of initial sub-quadratic growth, which then accelerates to quadratic growth in program size. However, he also found that in large discrete programs, the whole population can have the same fitness with $100 \%$ variety (all programs are non-identical). This phenomenon reduces selection pressure and may account for occasional observed instances of continued sub-quadratic growth.

The difficulty of the GP task may also have an effect on code growth. Gustafson et al. [28] tried to establish this relationship. They look at two different symbolic regression problems of different difficulties (by tuning the difficulty with different ephemeral random constant [ERC] ranges). They conclude that increased task difficulty leads to a higher selection pressure and less diverse genetic populations. Both of these contribute to an increased rate of code growth. They also make some recommendations with regard to controlling selection pressure to make similar tasks have lessened code growth.

Blickle and Thiele [10] investigated the causes of program bloat. They suggested that if reproduction rate was too low (and selection pressure correspondingly high) then redundancy free trees were prone to dying out and redundancy would grow. High fitness and highly redundant pro- 
grams spread exponentially through the populations.

Langdon and Poli $[46,45]$, go on to support Blickle and Thiele's claim that fitness based selection pressure causes solutions to grow in size. They claim that using a static evaluation method for fitness leads to larger solutions as there are simply many more larger solutions than smaller ones. [46] solely focused on mutation in order to show that even without crossover, using a fitness based selection process results in bloating.

There have been four theories that underly the causes of code growth in GP. Streeter [90] provides a good summary of these. These are the Intron Theory (introns are created as a response to destructive crossover), Diffusion Theory (that large programs propagate through a population quickly as they are more likely to have good fitness), Removal Bias Theory (inviable nodes occur deeper, so neutral crossovers tend to remove small trees from deeper points and then add larger trees) and Depth-Correlation Theory (which is similar to removal bias, but also adds that tree size itself is a defense from crossover and mutation). He then outlines resilience, a measure of how resistant to change in behaviour a program is (if mutations are made, how different is the programs behaviour). It is noticed that larger programs are more resilient. Finally, a modified selection method is used which adds in a penalty of having a low minimum behaviour change (MBC). This penalizes resilient programs that do not differ from parents very much. Having a MBC $=0.1$ is shown to eliminate code growth in his symbolic regression experiments.

Nordin and Banzhaf [58] use experiments on symbolic regression problems to argue that destructive crossover (that few crossover events increase fitness and may lower fitness) may lead to code growth, as code growth may protect programs from these destructive crossovers and mutations (Intron Theory).

Luke [49] uses experiments on symbolic and multiplexer tasks to show that if "inviable code" (redundancy) is actively inhibited or restricted from propogation in the populations, then this leads to an "increase" in tree 
growth. The paper goes on to claim that inviable code or introns are an effect of code growth and not the cause, effectively arguing against the Intron Theory school of thought. The paper then promotes the depthcorrelation theory to explaining code growth in GP.

Other works have suggested that there may be other pressures in GP that influence the growth of code in GP. McPhee and Miller suggest in [54] that there is a "force in GP that results in the development of more accurate replicators" (Replication Accuracy Force). That is that larger trees are more likely to yield semantically equivalent children to their parents via crossover. This "force" leads to a tendency of subtrees to grow in size. However, they also state in their work that some of their experiments did not exhibit this behaviour, meaning their conclusions are not universal.

Piszcza and Soule [64], demonstrate that while using GP to solve a symbolic regression problem, there is significant pressure for genetic robustness which forces the population away from high fitness (but less robust) solutions in favour of lower fitness (but higher in robustness) solutions.

Zhang and Soule [103] also work on the basis of introns and 'genes' which either do not influence fitness or have very little effect. Genetically robust solutions are solutions that are less likely to be degraded by genetic operators, such as crossover. They examine the role of genes that cancel each other out (common in redundant code). We find that allowing such canceling genes to exist in the populations leads to an overall increase in the rate of code growth, both through the inclusion of self-canceling code and through a general increase in introns. They also find that the GP evolution generally follows a two-step process. Initially the operative code evolves rapidly to achieve a (near) optimal fitness. Then, the inoperative code begins to evolve most rapidly to increase robustness. 


\subsubsection{Basic Bloat Control: Depth and Size Limiting}

The most basic method for controlling program bloating is to limit the size of the program tree. One way is to set a depth limit, where programs cannot grow larger (through the genetic operators) than a specified depth. Another way is to set a size limit, which limits the total number of nodes that a program can grow to. Both of these approaches are commonly used in GP.

Silva and Costa [82] compare two commonly used approaches to controlling bloat in GP: depth limits for trees and resource-limits for populations. Depth-limiting is the approach of specifying a limit to how deep a tree is allowed to be. Resource-limited GP is a technique which limits how many nodes a population of programs are allowed to have in total. That is, the total number of nodes that all the programs in a given GP population cannot exceed a specified amount. Static depth limiting and dynamic depth limiting (which modifies the depth limit dynamically) are tested, along with two dynamic resource limiting (normal and light) methods on three tasks: symbolic regression, even parity and artificial ant. They claim that resource-limited GP produces smaller and more accurate programs than the depth-limiting method.

\subsubsection{Parsimony Pressure}

Parsimony Pressure was one of the first major approaches forumulated to combatting program bloat. Parsimony Pressure is an indirect method of controlling bloat where the fitness function used in the GP system is modified to penalise larger programs. This results in a larger program being given a lower fitness value than a smaller program even if they perform equally well on the given task. By doing this, it is hoped that not only will there be selection pressure to find well performing solutions, but also to find smaller solutions. Some typical work on this method are reviewed in this sub-section. 
Early experiments by Koza [40] actually showed a loss in effectiveness when using Parsimony Pressure on a number of GP tasks. Works since then have attempted to improve the parsimony pressure methods/formulae.

Muhlenbein [99] treats GP as a statistical inference problem and applies the Bayesian model to formulate a class of fitness functions with both error and complexity terms (i.e. parsimony pressure). From this they develop an adaptive learning method that balances model-complexity to obtain smaller programs without loss of population diversity, which is needed for accuracy. It is only used on neural network synthesis tasks for experimentation although the authors claim it should work in other domains.

Nordin and Banzhaf [58] argues the need for "compression pressure" in all variable length evolutionary techniques (not just GP). They perform empirical experiments on a symbolic regression task and find that using pressure generally leads to a more efficient search and programs that are better at generalizing across unseen data. However, using pressure also tends to lead to premature convergence toward a non-optimal solution, that is, there is a degradation in performance of the resulting programs.

Soule et al. [87] agrees with other papers that parsimony pressure can produce poorer performance. It goes on to suggest (using results from experiments on the "even-parity problem" and using $f(i)=P(i) p(s i)$ for parsimony pressure) that parsimony pressure can drive a population quickly to minimal size and minimal performance. This is more likely with higher values of pressure. If this "trapping" does not occur, then GP trial shows little degradation. They also find that the relationship between size and performance in a population can give an indication of the trials chances of being trapped and failing.

Luke and Panait [50] describe a modification to the tournament selection operation which effectively performs parsimony pressure. If two randomly chosen programs in the tournament have the same fitness then the smaller program is selected. Two additional variants of this selection operator, which use direct-bucketing and ratio-bucketing, are also tested, where 
fitness is partitioned into buckets and programs are assigned to a bucket. Programs with identical bucket ranks are treated as if they have identical fitness in the parsimony pressure step. Experiments are performed on four tasks: artificial ant, 11-bit boolean multiplexer, symbolic regression and even-5 parity [40]. In $75 \%$ of their experiments, using lexicographic parsimony pressure or its variants maintains the same mean fitness as when using simple depth limiting. However, they find that their method cannot control growth in their symbolic regression problem and the code bloats. They claim that when using depth limiting in combination with lexicographic parsimony pressure, both equivalent fitnesses and capping of bloat can be attained.

\subsubsection{Other Bloat Control Methods}

Koza [40] describes using Automatically Defined Functions (ADFs) as an approach to redundant code. ADFs provide a mechanism for GP to encapsulate subtrees of code, allowing for reuse of "useful" portions of a GP program. These ADFs are regarded as functions during the GP evolution and are treated as atomic (i.e. unbreakable) subtrees. By using ADFs (which are only stored once and referred to multiple times), commonly used, "useful" pieces of code are more efficiently stored which saves computer memory. In a similar fashion, graph-based GP [65] exploits code re-use to improve memory utilisation.

Blickle and Thiele [10] attempt to identify redundancy and try to further explain the size problem in GP. They describe GP in a mathematically equivalent form and introduce a definition for redundancy using this form. This paper also introduces "marking" of program edges during fitness evaluation and only performing crossover on the marked edges (those edges which were used in the evaluation). This is to prevent "useless crossover" (crossover that would take place in unused portions of code). Performing experiments on three tasks (6-multiplexer, Truck back- 
ing and Artificial Ant [all from Koza [40]]), they find that when using marking that while some gains in program size could be made, performance largely depended on the problem type (they found no improvement on the artificial ant problem).

Nordin et al. [59] worked on the premise that introns exist in GP programs to protect highly-fit building blocks as well as protecting the entire, overall program from destructive mutations and crossovers (Intron Theory). Explicitly Defined Introns (EDIs) are introduced, which are nodes with integer values which determine the probability of a crossover point being selected at that node's position. EDIs provide GP with a mechanism for protecting parts of a program from the so-called destructive crossover. This means that redundant code growth should be reduced, as it is thought that redundant code serves as this protecting mechanism in standard GP. They suggest that using EDIs can improve fitness, generalization of programs and CPU time, though the improvement appears dependent on the range in which the EDIs are initialised. EDIs appear to replace normal implicit introns entirely in some cases (which can result in smaller programs), and work in tandom with implicit introns in other cases.

Ashlock et al. [5] introduces Single parent genetic programming (SPGP), which reduces bloat by doing subtree crossover only from trees within a fixed set of trees in the population (called ancestor trees). Analysis of the mean tree size growth in GP runs shows that SPGP "provides implicit control on tree size". Experimental tasks include: plus-one-recall-store, oddparity, plus-times-half and bio-informatic model fitting problems. They conclude that results and effectiveness of SPGP are reliant on the choice of ancestor trees and can vary widely.

\subsubsection{Simplification of GP Programs}

There have been a few past works which have tried using some form of program simplification to combat program bloat in GP, with varying re- 
sults. We look at a few of these in this sub-section.

Koza [40] describes an early simplification method, the editing function. This function provides a means of editing and simplifying the evolved programs that result from the GP system. He describes two uses for this type of function. The first is for cosmetically changing an evolved program in order to make the final programs more readable, without making any changes to the evolutionary run. The second use is to use the function during the evolutionary process in order to constantly simplify the GP programs in each generation. He tries this second use for the editing function on the boolean 6-multiplexor problem over 30 runs and discovers no improvements over the standard GP when using the editing function.

Hooper and Flann [33] provided one of the earliest attempts to use expression simplification to remove redundant GP code from programs. They used a set of over 200 rules to perform the simplification on programs, and introduced a simplification rate: the probability that a particular program in a generation will be chosen for simplification. They used this simplification method on a simple symbolic regression problem using a variety of rates. They found that using simplification led to programs that were significantly better performing than those produced by the standard GP. Also they found no significant increase in the run time of the GP system when using simplification, although experiments were only repeated 10 times for each system. They note that choice of simplification rate did not appear to have a profound effect on the simplification method's effectiveness.

Smith [85] evolves fuzzy decision trees (for use in data mining) using GP. In his approach, he uses a computer algebra to reduce the length on intermediate programs. He finds that this method is able to reduce the length of many of the programs, leading to accelerated convergence of the population. He uses this method in conjunction with a parsimony pressure method. More details are then given in later work [86].

Ekart [19] presents a simplification method using Prolog clauses which 
works on the LISP-S expression form of GP programs. This simplification method is used as a neutral mutation (explained as a form of mutation which alters the entity without affecting its ability). This simplification mutation is added as an additional genetic operator (along with crossover, mutation) and invoked at various frequencies with a given probability of being invoked on a program. Experiments are performed on a single symbolic regression problem and it is suggested that code growth can be moderated or even stopped without deterioration by using appropriate frequency and probability values. No suggestion is made on what the values should be.

Zhang et al. [101] describes a more complex program simplification approach that uses simple algebraic techniques, prime numbers and hashing techniques. By using prime numbers to form an encoding for a program tree, this method allows for more complex forms of simplification (beyond the realm of basic algebraic rules). This approach is examined on four object classification problems of increasing difficulty. The results suggest that using their simplification approach is more efficient and more effective than the basic GP approach without simplification.

Although relatively few in number, these related works show some promise in using simplification to make the GP evolution more efficient and possibly more effective. Accordingly, in this thesis we will consider developing our own simplification system which will be applied to programs during the GP evolution, and compare the performance with the standard GP, using a variety of regression and classification tasks.

\subsection{Related Work to Building Blocks}

Conceptually, we may regard building blocks as simply small components that can be formed into larger, more fit components through the use of genetic operators. In this section, we examine works related to building blocks and building block theory in Genetic Algorithms (GAs) and GP. 


\subsubsection{Building Block Hypothesis and Schema Theory in GA}

The concept of building blocks (small pieces of genetic structure which contribute much to fitness) can be traced back to Genetic Algorithms (GA), where it was hypothesised by Goldberg [25] in his building block hypothesis that "Short, low order, and highly fit schemata are sampled, recombined [crossed over], and re-sampled to form strings of potentially higher fitness".

In the 1970s, Holland [31] presented the argument that GAs perform a parallel search for certain patterns of chromosomes (rather than a search through the solution space), which he called schema (also known as schemata). Goldberg [25] continued this idea in his formulation of the building block hypothesis $(\mathrm{BBH})$. These ideas proposed that GAs were capable of hierarchically constructing solutions by constructing smaller blocks, and then assembling these smaller blocks progressively into a solution. It is still a debated topic over whether the $\mathrm{BBH} /$ schema theory is valid and whether it truely explains how GAs are able to construct such solutions of high fitness, though it is generally highly regarded as the process that GA utilises to solve given problems [32, 25, 22], and why it is so powerful in doing so.

\subsubsection{Building Block Hypothesis and Schema Theory in GP}

Ever since the creation of GP, there have been several attempts to bring a similar building block theory to the realm of GP $[40,4,94,70,60,76]$. These attempts have themselves spawned several approaches to describing building blocks: a subtree to a solution tree [3], a rooted subtree [78], a block of code [34]. O'Reilly and Oppacher's early work [61] developed a schema theory for GP and pointed out the difficulty in formulating a corresponding building block hypothesis for GP.

Current schema theory works on the basis that schemas of small order and high fitness grows in GP populations exponentially. Formulating a good schema theory for GP is made more difficult by the increased flexibility with which GP programs can be combined together, along with 
having a variable representation (which can make calculating probabilities of things such as survivability of schemata very complex). A number of exact schema theory formulae have been developed for some specific GP setups [67, 68, 69, 73, 74, 75, 71] (using one-point crossover, headless chicken crossover etc.) which are able to make more accurate predictions of the frequency of schemata from generation to generation. However, many of these schema theories are for limited/special forms of GP and are not applicable to the standard GP. Furthermore, these schema theories usually can only make estimates for one generation ahead, and cannot reliably predict whether good schemata will propagate during a GP run.

It has been argued by some researchers that because rooted subtrees (those that exist from the root of the program tree) have maximum impact on the behaviour of the program tree and that they are the only subtrees useful to track and analyse. Concentrating solely on rooted-subtrees also reduces the complexity of schema theories tremendously [71, 78]. However, recent work has used both general subtrees $[52,81]$ and rooted subtrees/structures as models for building blocks [80,79], giving no clear consensus on which is more "useful" to analyse.

Daida et al. [14] looks at the simplified analysis of single-node subtrees in order to help determine what a building block is. It concentrates on tracking ephemeral random constants (ERCs) in a symbolic regression task $\left(f(x)=(x+1)^{3}\right.$ with fitness points $\left.[-1,0)\right)$. The first experiment changed the range at which ERCs were generated in at the beginning of GP evolution (used No ERCs, $[-1,1],[-10,10]$ and $[-100,100])$ ). It was found that the larger the chosen range is above $[-1,1]$, the more difficult it was for GP to find a solution. They find that both the content and context of these ERCs have an affect on how the GP system performs. They do not however come to a full conclusion on what a building block is. They conclude that ERCs do exhibit the traits in the intuitive definition of a building block: "simple components out of which more complex things can be made". They also suggest that a building block in GP is different to that in 
biology (a comparison that has been suggested by other researchers), and contend that "the assumption of a GP parse tree as a biological analog for real-world DNA may be tenuous".

In other work [16], they probe into whether there are limits to which building blocks can be mixed and assembled by a GP system. It utilizes a GP task they call the Highlander Problem. This problem tries to assemble a program which contains a high fraction of unique nodes which are from the initial population. This is claimed to be tunably difficult, that is the difficulty of the problem is dependent on what the target fraction is. The paper claims that only $2-18 \%$ of all nodes in the initial population make it into the final solution, and that there are three main processes during a GP run: recombination, selection and aggregation.

\subsubsection{Empirical Analysis}

Relatively few works have been performed which use empirical analysis to support their claims. A large number of works have focused on schemas, which can be both fairly complex and very numerous. This makes empirical analysis of schemas intractable for any "interesting" problems, as the number of possible schemas can be tremendously large. Even so, there have been some empirical studies of schema and building blocks.

Poli and Langdon, [70, 71] tracked hyperschema in a very restricted environment, using only a population of 50, depth limits of three or four and not using genetic mutation. It was found that the crossover operator maintained diversity within the populations, both being destructive and constructive of schemas within the GP populations.

Langdon and Banzhaf [44] analysed schema within GP programs which performed best in their runs for two problems. They found that these solution programs often contained fairly large repeated patterns.

Wilson and Heywood [95] analysed linear GP programs, looking for repeating blocks of instructions. They suggest that the existence of modules 
that are reused by linear GP programs may exist in the populations.

Majeed [52] analysed subtrees of fixed depth in GP runs, tracking only those subtrees which occurred in over half the previous generations programs. While analysis on these subtrees did not yield solid conclusions, it was found that the fitness of a particular subtree in one run was independent of its fitness in other runs, meaning that fitness of a particular subtree was not consistent amongst all the runs.

Smart et al. [84] develop a novel way of representing building blocks within GP using so-called tree-fragments. Their early analysis shows that there is a large variation in the number of fragments in GP evolution, and a non-monotonic growth of the most "useful". They plan to perform more analyses in future work.

Empirical studies of building blocks largely use a restricted environment (e.g. low population, low depth, only looking at best performing programs or select programs). In this thesis, we aim to be able to track all building blocks of a particular form in all generations of individual GP runs. No previous work has focused on the effects of removing program redundancies on the GP populations, which is another aim of this thesis.

\subsection{Related Work to Fitness Evaluation}

Fitness evaluations is the most computationally costly operation in Genetic Programming (GP) [24]. In each generation, all programs typically need to be evaluated for fitness, which is then used by genetic operators (e.g. genetic selection, reproduction). In many tasks (such as classification) the number of fitness cases available can be in the hundreds or even thousands in number. This directly translates into evaluating a single program hundreds (or thousands) of times in order to determine that program's fitness (or classification accuracy).

There have only been a few major approaches to reducing the fitness evaluation costs in GP. The first of these approaches is to reduce the cost of 
fitness evaluations by refining the set of fitness cases that is presented to the GP system. By only presenting the GP system with a carefully selected subset of fitness cases, fitness evaluation costs can be reduced without loss of training effectiveness. Giacobini et al. [24] use statistical methods to construct a task independent approach to determining the number of fitness cases required to reliably (given a confidence interval) reconstruct a target function.

Gathercole and Ross [23] present three of their own dynamic fitness case subset selection methods, which select a subset of the fitness cases for GP to use during fitness evaluation. These three methods are dynamic subset selection (adjusting the set each generation to include "difficult" cases encountered so far), historical subset selection (selecting "difficult cases from previous GP runs) and random subset selection (selecting a subset at random each generation). They find that using dynamic subset selection can produce better results than the standard GP, while taking significantly less time to perform their problems. Both historical and random selection methods are only able to approach or perform worse than the standard GP.

Zhang and Cho [98] also present a dynamic fitness case subset selection method, though in their approach, they incrementally increase the training set size as the evolution continues (Incremental Data Selection). They find that using their method to reduce the number of fitness cases can lead to reductions in node evaluations without loss in generality of the evolved programs, especially in GP tasks with large numbers of fitness cases.

A second approach has been to improve the fitness evaluation procedure directly. One such method has been to evaluate fitness cases in parallel $[13,29,37]$. Instead of evaluating each fitness case individually, which requires parsing a GP program tree for each evaluation, this method evaluates all the fitness cases during a single GP program parsing. Keijzer [37] describes this as "Vectorised Evaluation".

Using vectorised evaluation, all of the fitness cases are joined together 


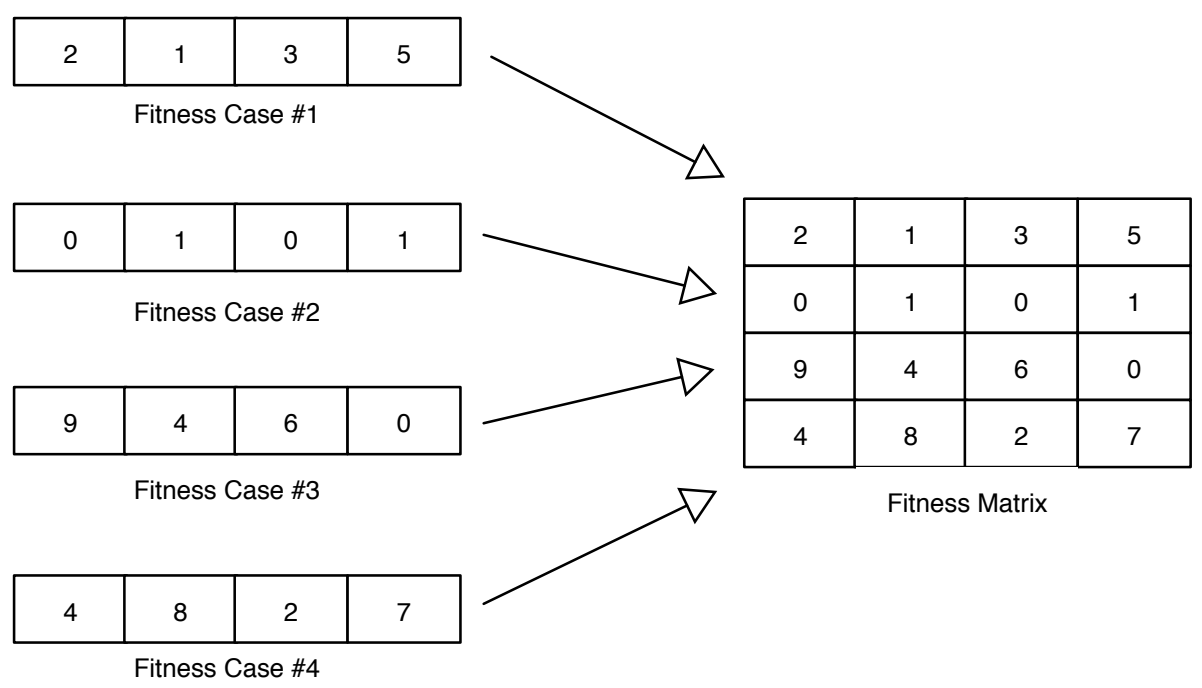

Figure 2.4: Example of fitness case vectors being joined into a single fitness matrix.

to form a matrix (see figure 2.4). Now when fitness evaluation is performed on a GP program, each node in the program is presented with the entire set of fitness cases (in matrix form). Instead of a single evaluation result being stored in each node, a vector of results (one for each fitness case) is kept. Using this approach, the entire vector of fitness case evaluation results is calculated using only a single parsing of the GP program (which can often be costly). By processing the fitness cases in parallel, the entire fitness evaluation component of the GP system is improved greatly.

[29] and [13] exploit this vectorised evaluation method further, by using a Graphics Processing Unit (GPU) to perform the fitness evaluation. GPUs are used to process 3D graphics and textures (often for computer video games) and are highly specialised for vector operations. By converting the fitness case matrix into a graphical texture (which are in themselves matrices), these two papers used the GPU texture shaders to perform vector operations on the features (transforming them into the desired fitness case results) at very high speeds. They found that when presented with 
a large number of fitness cases, the gain in evaluation speed highly outweighed the overhead introduced by converting fitness cases into GPU textures.

A majority of these previous works in GP have fallen into these two approaches, and only a few have attempted a third approach: lowering evaluation costs by reducing the number of node evaluations that need to be performed.

The method described by Jackson [36] avoided some fitness evaluations by not evaluating programs produced by the so-called fitness-preserving crossover. By marking nodes as they are used for evaluation, portions of a program that were not used at all for the fitness cases could be identified. If crossover occured within one of these portions, the resulting program would have the same fitness as one of the parents.

Another method in Xie et al. [97] describes an approach to reducing this cost by dividing the population into clusters, based on the outputs that the programs give on a small number of fitness cases. Evaluations were performed on only a single program in each cluster, with the other programs in the cluster being assigned the same fitness. Experiments were performed on a symbolic regression task, a binary classification task and a multi-class classification task. It was found that using this approach led to a significant reduction in the number of programs that were evaluated, although the reduction in the CPU time was small.

Work performed by Keijzer [37] uses two subtree caching methods that are based around using a fixed cache size, in conjunction with vectorised fitness case evaluation. The cache is maintained to try to ensure that the most beneficial subtrees are present in the cache during GP runs. Another work by Roberts [77] uses a hash table cache with hash buckets, with the aim of hashing each subtree uniquely. Seldom used subtrees were removed from the cache to reduce overhead from linearly searching the buckets. This caching mechanism was used in a typed-GP system for experiments on image segmentation problems. Results from both these 
works suggest that using caching can potentially lead to a large reduction in the number of node evaluations performed during GP runs.

\subsection{Chapter Summary}

In this chapter we have presented an overview of related concepts (e.g. machine learning, evolutionary computation, genetic programming) and presented related work to the focus areas of this thesis: program bloating, building blocks in GP and improving fitness evaluation. A summary of the current state is as follows:

- Existing methods of controlling program bloat (e.g. edge marking, parsimony pressure, EDIs) have shown mixed results, and can either perform poorly for some tasks, or across the board. Program Simplification has shown some promise in past work, but most require translation into other forms in order to perform simplification. In this thesis, we want to develop a new program simplification method which will work directly on the GP programs in the populations.

- While building blocks in GP has had much attention in the theoretical domain, there has been little work which performs empirical analysis of building blocks in GP. Existing work has usually been performed using heavily restricted environments, and no work has tried to analyse the effect that program simplification can have on GP populations. In this thesis, we will analyse and track building blocks through GP runs in order to investigate the effects of program simplification on the GP populations.

- Reducing the cost of fitness evaluation in GP has mainly fallen into two areas: refining fitness cases and directly improving the fitness evaluation method. However, few attempts have been made to reduce the number of node evaluations that fitness evaluation needs 
to perform to evaluate the GP population. In this thesis, we will develop methods of reducing the number of node evaluations that fitness evaluation performs during the course of a GP evolution. We expect that reducing the number of node evaluations will also reduce the amount of time that GP requires to evolve a solution. 


\section{Chapter 3}

\section{Datasets}

Experiments performed in this thesis are made using a variety of tasks from several domains. These include symbolic regression, image classification and medical classification, tasks. The tasks are also of varying difficulties to enable us to test our new algorithms and methods over a wide range of data. The experiments performed in each chapter make use of one or more of these tasks, which we describe in this chapter.

\subsection{Symbolic Regression}

Symbolic Regression [40] is the process of inducing a mathematical function from a set of data. It is termed "symbolic" to emphasise that the process searches for a symbolic description of a model (along with a set of parameters) and not simply a set of parameters for a pre-selected model (as is the case with processes such as neural networks or many statistical parameter regression methods). It is a commonly used task in evolutionary computation in part because it is easily interpretable and can be tuned to provide different degrees of difficulty.

In GP, the system is presented with a set of data points, from which GP is required to form a program which can "fit" the data as closely as possible. The typical fitness function used to guide this task is Mean Squared 
Error (MSE), which is defined as follows for $n$ data instances:

Fitness $($ Program $)=M S E($ Program $)=\frac{1}{n} \sum_{i=1}^{n}\left(\text { Output }_{i}^{\text {Program }}-\text { Output }_{i}^{\text {Data }}\right)^{2}$

That is, MSE is the squared sum of the differences between the program output and the target output (determined by the labelled data) for each of the instances in the data set. This means that a lower MSE value is desirable, with the optimal value being 0 (meaning the program fits the data perfectly).

In this thesis, we use several regression problems (tailored to suit the targetted experiments):

Regression $_{1}$ The first problem is a simple function: $f_{1}(x)=8 x+8$, and was selected as a problem that requires repetition (as solutions would need to piece together eight feature terminals) in the solutions found by GP. For this regression problem, the dataset consists of 400 points, taken at 0.05 intervals between -10 and 10 . This function is displayed in figure 3.1(a).

Regression $_{2}$ The second regression problem is a more difficult Binomial problem: $f_{2}(x)=(x+2)^{3}$. It was selected to be a difficult symbolic regression problem for GP to solve, while still having many possible solutions (e.g. GP forming $(x+2)^{3}$ vs. forming $(x+2)\left(x^{2}+4 x+4\right)$. The dataset for this problem consists of 200 points, taken at 0.1 intervals between -10 and 10. This function is displayed in figure 3.1(b).

Regression $_{3}$ The third regression problem is a more difficult, piecewise function:

$$
f_{3}(x)= \begin{cases}x^{3}-5.8 x+3.0 & x<0 \\ \frac{x-12.0}{x^{2}}+1.0 & x \geq 0\end{cases}
$$

It was selected to be a very difficult problem for GP to solve, and would require the $i f<0$ function in order to form a solution. The dataset for this 


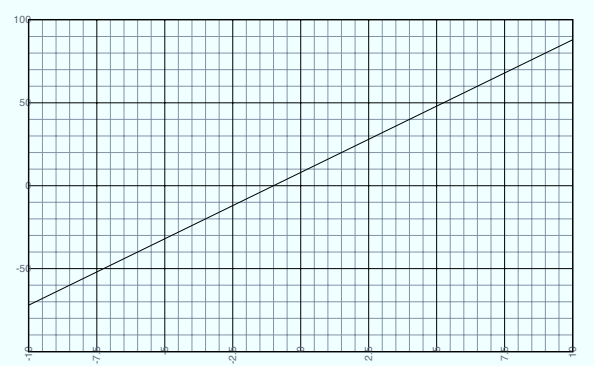

(a) $f_{1}(x)=8 x+8$

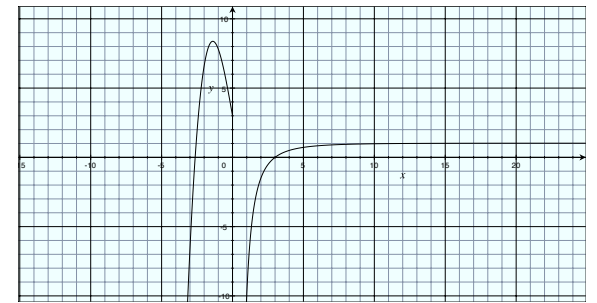

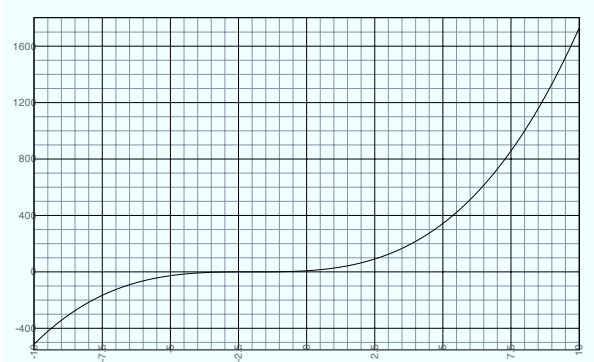

(b) $f_{2}(x)=(x+2)^{3}$

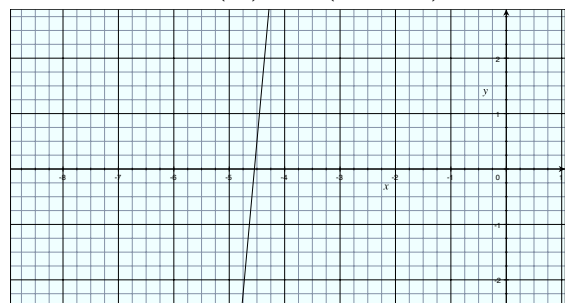

(d) $f_{4}(x)=11 x+50$

(c) $f_{3}(x)=x<0 ? x^{3}-5.8 x+3.0: \frac{x-12.0}{x^{2}}+1.0$

Figure 3.1: Plots of the four symbolic regression tasks: (a) Regression ${ }_{1}$, (b) Regression $_{2}$, (c) Regression 3 , (d) Regression 4 .

problem consists of 200 points, taken at 0.1 intervals between -10 and 10 . This function is displayed in figure 3.1(c).

Regression $_{4}$ The fourth problem is another simple function: $f_{4}(x)=$ $11 x+50$, and consists of 200 points, taken at 0.10 intervals between -10 and 10. This function is displayed in figure 3.1(d). This function is used in our building block analyses in chapters 5 and 6 .

\subsection{Image Classification}

Image Classification is the process of attributing images to one of a set of pre-specified class labels. It has wide applications including analysis of satellite imagery, handwriting recognition, facial recognition.

A solution to a classication task would involve a program that can correctly map an input vector describing an instance (an object, an image, 
an example item etc.) to one of a small set of class labels (e.g. "square", "circle", "heads", "tails"). Writing such computer programs is difficult, time consuming, and often infeasible: human programmers are often unable to identify all the subtle and interrelated conditions that are needed to distinguish between the different classes.

In GP, image classification is typically performed by extracting a number of image features from the images themselves. These are numeric values which represent characteristics in the images (e.g. mean intensity, key point positions, edge pixels). These features are used by the GP system to develop programs which will perform the classification given values for these features. The fitness function used to guide the evolution is the classification accuracy that the GP program can obtain on the set of data it is being trained on. That is, for a training set of $n$ classification instances:

Fitness(Program $)=\frac{1}{n} \sum_{i=1}^{n} \begin{cases}1 & \text { TranslatedOutput }_{\text {Program }}^{i}=\text { Output }_{\text {Data }}^{i} \\ 0 & \text { else }\end{cases}$

Because standard GP programs output a numeric value themselves, this output needs to be interpreted to indicate a specific class. In this thesis, we use the simplistic static class translation method [100] which involves splitting the output into intervals; each of which represent a single class. While this may not be the optimal method of translating the GP output into classes, finding an optimal method is beyond the scope of this thesis. An example of static class translation would be:

$$
\text { TranslatedOutput } t_{\text {Program }}^{i}= \begin{cases}\text { Class1 } & \text { Output } t_{\text {Program }}^{i} \leq-1.00 \\ \text { Class } 2 & -1.00<\text { Output }_{\text {Program }}^{i} \leq 0.00 \\ \text { Class3 } & 0.00<\text { Output }_{\text {Program }}^{i} \leq 1.00 \\ \text { Class4 } & 1.00<\text { Output }_{\text {Program }}^{i}\end{cases}
$$

In this thesis, we use two image classification problems of different difficulty: 
Shapes This dataset consists of 1800 image cutouts of larger white circles, small grey squares and a noisy background. Each fitness case for this dataset is made up of 5 extracted image features from these image cutouts.

- Area: The image is segmented using thresholding, and the number of white pixels is summed up to obtain this feature.

- Perimeter: Again, the image is segmented by using thresholding. Subsequently, the Sobel edge detection convolution mask is applied to obtain the edge pixels. The number of white edge pixels is summed up to obtain this feature.

- Thinness: This feature is obtained from the previous two and is de-

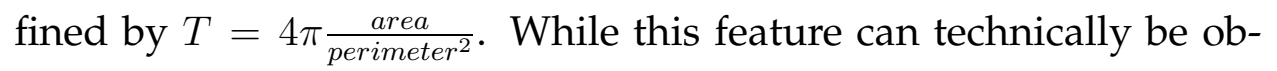
tained by GP from using the first two features, it is particularly useful in determining the difference between squares and circles and is therefore valuable to include in the feature set and not depend on GP to construct on its own.

- Mean: This is obtained by taking the average value of all the pixels in the image.

- Standard Deviation: This is obtained by calculating the standard deviation value of all the pixels in the image.

In total there are three classes: white circle, grey square and noisy background. The similarity between the grey squares and the noisy background makes the classification problem reasonably difficult. It is important to note that the features we use for this dataset are extracted from $16 \times 16$ pixel cutouts of each of the shapes in each larger image. Examples of cutouts for each of the classes is given in figure 3.2, while an example of a shapes image is shown in figure 3.4 (a).

Coins This dataset consists of $48070 \times 70$ pixel image cutouts of New Zealand 5 and 10 cent coins with different sides up and different orientations. Each instance is described by 8 extracted image features, which are 

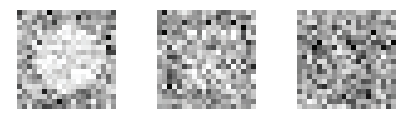

(a) (b) (c)

Figure 3.2: Example $16 \times 16$ pixel cutouts of shapes taken from images in the shapes dataset. (a) White Circle, (b) Grey Square and (c) Background

found by taking the mean and standard deviation of the pixel values in four regions of each cutout. In total there are four distinct classes: 5 cent heads, 5 cent tails, 10 cent heads, 10 cent tails. An example of a cutout of each class is given in figure 3.3, while an example of a larger coins image is shown in figure $3.4(\mathrm{~b})$.

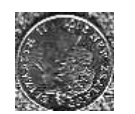

(a)

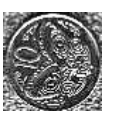

(b)

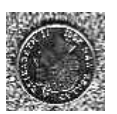

(c)

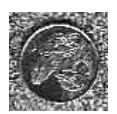

(d)

Figure 3.3: Example $70 \times 70$ cutout of a coin taken from images in the coins dataset. (a) 10c Heads, (b) 10c Tails, (c) 5c Heads and (d) 5c Tails.

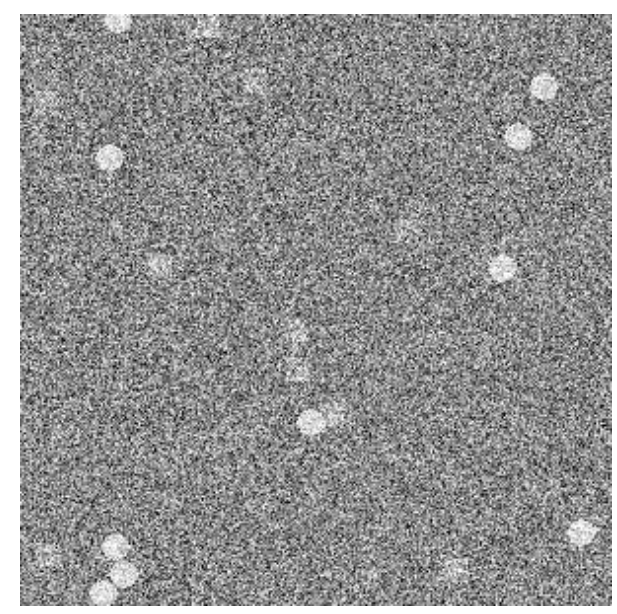

(c)

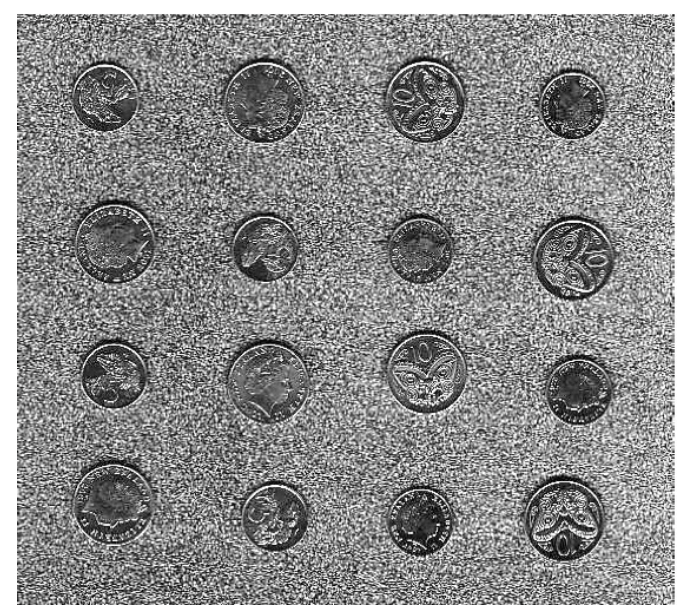

(d)

Figure 3.4: Example images from the: (c) Shapes dataset, (d) Coins dataset. 


\subsection{Medical Classification}

Medical classication tasks arise in a wide variety of practical situations. Diagnosing medical conditions from medical imaging and determining whether a breast cancer case with a number of attribute values belongs to "malignant" or "benign" from a large data set are just two examples of the many important medical classication tasks. In many cases, highly trained experts (experienced doctors) are able to perform these classication task well, but there is either a shortage of such experts, or the cost of the experts is too high, so performing these tasks by hand is too expensive and/or too slow. Given the amount of medical data that needs to be classified, computer based solutions to many of these tasks would be of immense social and economic value.

The fitness function is the same general classification fitness function we use in Image Classification.

$$
\text { Fitness (Program })=\frac{1}{n} \sum_{i=1}^{n} \begin{cases}1 & \text { TranslatedOutput } i_{\text {Program }}^{i}=\text { Output }_{\text {Data }}^{i} \\ 0 & \text { else }\end{cases}
$$

In this thesis, we use two medical classificaion problems of different difficulty:

Wisconsin Breast Cancer This dataset is taken from the UCI Repository of Machine Learning Databases [6] and consists of 699 cases of breast cancer diagnosis data. The instances are described by 9 integer valued attributes between 1 and 10, which describe the following: Clump Thickness (CT), Uniformity of Cell Size (USz), Uniformity of Cell Shape (UShp), Marginal Adhesion (MA), Single Epithelial Cell Size (SESz), Bare Nuclei (BN), Bland Chromatin (BC), Normal Nucleoli (NN), and Mitoses (M). For this dataset there are only two classification outcomes: benign or malignant. 


\begin{tabular}{|c|c|c|c|c|c|c|c|c|c|}
\hline CT & USz & UShp & MA & SESz & BN & BC & NN & M & Diagnosis/Class \\
\hline 5 & 1 & 1 & 1 & 2 & 1 & 1 & 1 & 1 & benign \\
1 & 1 & 1 & 1 & 2 & 1 & 2 & 1 & 1 & benign \\
10 & 8 & 8 & 2 & 8 & 10 & 4 & 8 & 10 & malignant \\
1 & 4 & 3 & 10 & 4 & 10 & 5 & 6 & 1 & malignant \\
\hline
\end{tabular}

Table 3.1: Example instances from the Breast Cancer Wisconsin Dataset

SPECT heart data This dataset is also taken from the UCI Repository of Machine Learning Databases [6] and consists of 267 instances of diagnoses derived from Single Proton Emission Computed Tomography (SPECT) images. Each image was processed to form 44 continuous features, and then further processed to form 22 binary features which are used to describe each fitness case. Again, there are only two classification outcomes: normal and abnormal.

\begin{tabular}{|c|c|c|c|c|c|c|c|c|c|c|c|c|c|c|c|c|c|c|c|c|c|c|}
\hline F1 & F2 & F3 & F4 & F5 & F6 & F7 & F8 & F9 & F10 & F11 & F12 & F13 & F14 & F15 & F16 & F17 & F18 & F19 & F20 & F21 & F22 & Class \\
\hline 1 & 1 & 1 & 1 & 1 & 1 & 0 & 0 & 1 & 0 & 1 & 0 & 1 & 0 & 1 & 1 & 0 & 0 & 1 & 1 & 0 & 1 & normal \\
0 & 1 & 0 & 0 & 0 & 0 & 0 & 0 & 0 & 0 & 0 & 0 & 1 & 1 & 0 & 0 & 1 & 0 & 0 & 1 & 0 & 0 & normal \\
0 & 0 & 0 & 1 & 0 & 0 & 0 & 0 & 1 & 0 & 0 & 0 & 0 & 0 & 1 & 0 & 0 & 0 & 0 & 1 & 0 & 1 & abnormal \\
1 & 0 & 0 & 0 & 1 & 0 & 0 & 0 & 0 & 0 & 0 & 0 & 0 & 0 & 0 & 0 & 0 & 0 & 0 & 0 & 0 & 0 & abnormal \\
\hline
\end{tabular}

Table 3.2: Example instances from the SPECT Heart Dataset

Because both of the medical classification datasets that we use are $b i$ nary classification problems, we use a slightly different class translation method for converting the GP output into a class. As there are only two classes, we can simply set a single boundary to separate the two classes. We use zero as this boundary, setting values less than zero to mean one class (e.g. benign or normal) and any values greater than or equal to zero to mean the other class (e.g. malignant or abnormal). 


$$
\text { TranslatedOutput } t_{\text {Program }}^{i}= \begin{cases}\text { Class1 } & \text { Output } t_{\text {Program }}^{i}<0.00 \\ \text { Class2 } & 0.00 \geq \text { Output }_{\text {Program }}^{i}\end{cases}
$$

\subsection{GP Settings}

\subsubsection{Common Parameters}

Here we describe the common parameters that are used in a typical GP system.

Population Size: This parameter controls how many programs each generation contains. A typical setting for this parameter may be 500 to 1000 programs per generation.

Generations: This controls how many generations of evolution are executed before the GP system is halted and a solution program is given. Typically, this parameter will be set to around 50 to 100 .

Terminal Set: The terminal sets consist of the feature terminals described earlier for each task, as well as a number of randomly generated floating point number valued numeric terminals.

Function Set: The function set usually consists of the four arithmetic operators and a conditional operator. In our experiments (unless specified otherwise) we use the function set $\{+,-, \times, \div$, if $<0\}$.

Program Generation: As is common practice, we use the ramped halfand-half method to generate the initial population of GP programs.

Selection: This controls how GP programs are selected for the various other genetic operators. We used a fitness-proportionate selection method.

Crossover, Mutation, Reproduction Rates: These three parameters control what proportion of the program population in each generation will be formed by Crossover, Mutation or Reproduction. These three settings must of course add up to $100 \%$. Common values for these settings are $60 \%$ Crossover, $30 \%$ Mutation, and 10\% Reproduction, that is, crossover rate is 
usually higher than the mutation rate, and reproduction rate is typically low.

Minimum and Maximum Tree Depths: These parameters control how small and how large a program tree can grow to. Any resulting tree from the genetic operators are not allowed to be lower than the minimum depth or larger than the maximum depth. Typical settings may have a minimum depth set at 3 , and a maximum depth set at 6 .

It is important to mention that the terminals, functions, fitness functions, crossover rates, etc. used in this thesis are almost certainly not the best values to use for each task. However, seeking the best values is beyond the scope of this thesis. This thesis is focused on investigating methods of removing redundancy and reducing fitness evaluation cost.

\subsubsection{VGP and Machine Environment}

The GP system that we use in our experiments for this thesis is Victoria Genetic Programming (VGP) [83], written by Will Smart at Victoria University of Wellington. It is written in the $C$ programming language and is designed for high execution speed over modularity.

All timed experiments (those requiring CPU time to be recorded) were performed on a single machine so that the relative timings would be consistent. Since the experiments in different sections of this thesis were performed at different periods and with different random seeds, experiments are generally not directly comparable between chapters as they have been performed on different hardware. However, for each set of results, we have re-run tasks using the standard GP, so that we may make a valid comparison. A large number of the experiments were performed on a Dell Optiplex GX745 computer with the following specifications: 


$\begin{array}{cc}\text { CPU } & \text { Pentium D 2.8Ghz } \\ \text { Chipset } & \text { Intel Q965 (ICH8) Express Chipset } \\ \text { RAM } & \text { 2048MB DDR SDRAM } \\ \text { Network } & \text { Broadcom 5754 Gigabit Ethernet LAN } \\ \text { HardDisk } & \text { 80GB Serial ATA 7200rpm disk } \\ \text { OS } & \text { NetBSD }\end{array}$

Other experiments which did not require timing measurements and specifically those that were computationally expensive and time consuming were distributed to multiple (typically 20 to 30) machines with different hardware configurations. 


\section{Chapter 4}

\section{Algebraic Simplification of GP Programs}

\subsection{Introduction}

One of the current problems in genetic programming (GP) is that of code bloat (see section 2.2, page 18). Genetic programs tend to grow very quickly in size, easily out-pacing the improvements in program fitness as evolution progresses. This is because increasing amounts of code being formed is often redundant and does not provide any contribution towards the program's fitness measure. As a simple example of redundant code, consider the program $(+x(-y=y))$. The $\left(\begin{array}{lll}-y & y\end{array}\right)$ subtree can be regarded as redundant, as it provides no more functionality than if the subtree were removed. Programs containing a lot of these redundancies can be both inefficient in execution and harder to understand. As the programs grow rapidly, they can quickly exhaust a system's available resources, slow down the evolution process, and even halt the system before a "good" solution is found. This may limit GP's ability to scale to more complex problems requiring larger-sized solutions.

Simplification is one approach to combating code bloat. Simplification works by directly processing and removing redundant code from pro- 
grams within a GP system. The editing function proposed in [40] is an example of a simplification component which intends to improve comprehensibility and execution efficiency of the final solution at the end of the evolutionary process. However, since code bloat is a problem that occurs throughout the evolutionary process, online simplification can be invoked during the GP evolution. Simplification has been tested on several symbolic regression [33, 19] and multi-class classification [101] tasks. These early results have shown that in most cases, simplification can improve the efficiency of the GP system, producing smaller sized programs and requiring shorter training times to find solutions. It has also been found that certain GP systems utilising simplification are capable of producing more effective/accurate solutions than the standard GP without using any form of simplification.

\subsubsection{Chapter Goals}

In this chapter, we want to develop a program simplification method for applying to GP programs during the evolution process, in order to remove redundancies from the programs. More specifically, we aim to investigate the following issues:

- how the online simplification algorithm can be constructed by combining some algebraic simplification rules and hashing techniques;

- whether the simplification improves the system efficiency of the evolutionary process; and

- whether this approach deteriorates the performance compared with the standard GP method without simplification. 


\subsection{Algebraic Simplification}

In the standard GP system, programs are represented as a LISP-S (or similar language) expression, which is stored in a tree representation [40]. Terminal nodes consist of either numerical constants (e.g. 0.56, -0.12) or features/variables. Functions usually consist of mathematical functions/operators, such as,,$+- \times, \div, \sin$, as well as conditional operators such as if $<0$, if $>0$. Based in this setting, a genetic program is essentially equivalent to an algebraic expression.

The Algebraic Simplification method makes use of this idea to simplify genetic programs online (during the GP evolution process).

The central aim of any simplification method is to obtain a smaller program, by removing the redundancy of a program, that yields the same output (or at least very very close) as the original program. In this approach we use algebraic simplification in the form of simplification rules which are applied to programs in a postfix manner. Also key to our approach is the use of hashing to estimate the algebraic equivalence of two program (sub)trees. This is a very useful property to be able to quickly test for.

In the rest of the section, we describe the simplification rules and the simplification process, then we will give an example to show how a typical GP program can be simplified, and finally summarise the whole simplification algorithm.

\subsubsection{The Simplification Rules}

In algebraic expression simplification, there are many simplification rules that can be applied (where appropriate) to a given algebraic expression. These are basically mathematical equivalences that say that these two expressions (e.g. $x+x+x$ and $3 x$ ) are equivalent in function. In simplification, we would take the shorter of the two equivalent expressions.

Similarly, in our approach we also use multiple simplification rules, 
each of which may only be suitable for removing a particular part of the genetic program. Since we are always wanting the shorter of the two equivalent expressions, we can split our rules into two parts: a precondition and a postcondition (similar to STRIPS operators [21]). The precondition represents the state of the surrounding nodes in the program tree that must be present in order to be able to apply the simplification rule, and the postcondition represents the additions and deletions made to the program tree to obtain the shorter, simplified form.

These rules form the ruleset of the program simplification algorithm, which has been developed to cover the major sources of redundancy in the evolved genetic programs. For example,

- an arithmetic operator with only constant children (e.g. $\left(\begin{array}{ll}+ & 2\end{array}\right)=$ 5)

- subtraction or division of self (e.g. $\left.\left(\begin{array}{lll}-f 0 & f 0\end{array}\right)=0\right)$

- redundant conditionals, where the outcome is always the same (e.g. if $<0(2 \mathrm{fO} f 1)=\mathrm{f} 1)$

The simplification rules used in this approach are presented in table 4.1. In this table, we use lower-case characters to represent constants (e.g. $a, b, x, j)$, and upper-case characters to represent variables (e.g A, B, X, J). A complete list of the simplification rules that we use in our method are given in table 4.1 .

\subsubsection{The Simplification Process}

To apply this ruleset to a genetic program for simplification, we used a kind of recursive "greedy" engine. It recursively travels through the program tree in a bottom-up fashion by the postfix traversal order. For each node it processes, the algorithm checks the precondition for each simplification rule in the ruleset. If a rule matches, it is applied to the partial tree 
Table 4.1: The simplification rules used.

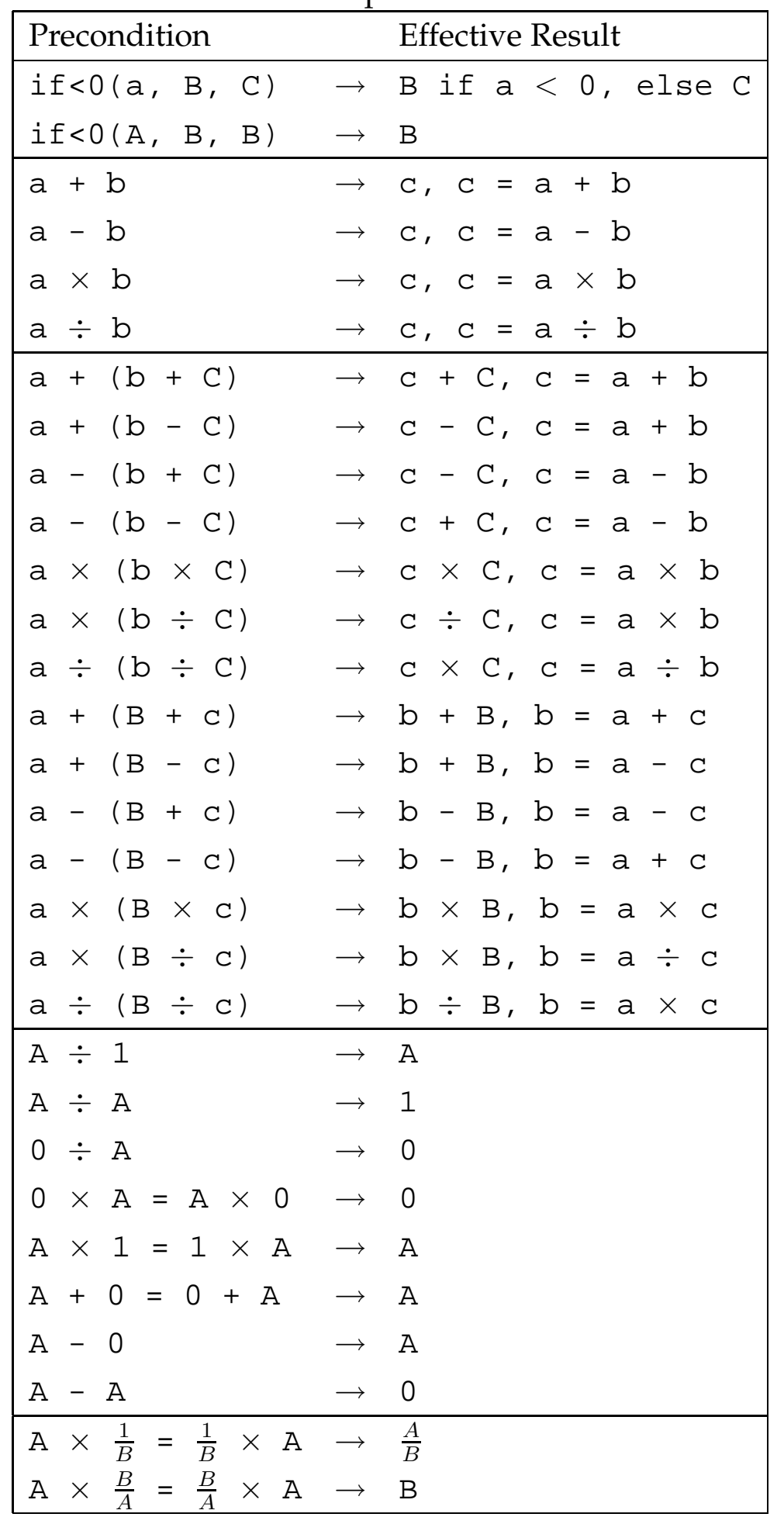


associated with the node to make simplification. If none of the rules can be applied at a node, the algorithm moves to the next node (either the other child node or the parent node).

In this way, the algorithm guarantees that each node in the program tree is only visited once. However, as all simplification rules only look at a static and limited area, arbitrary depth levels of simplification (simplification of terms which are not neighbouring but far away) are not supported in this algorithm. This means that more complex forms of redundancy, where the cancelling portions of the program tree are separated, cannot be removed by our approach. However, the simplicity of the greedy engine and the simple rules allows for the simplification to work fast, which minimises the overhead added to GP by using simplification.

\subsubsection{Estimating Algebraic Equivalence using Hashing}

An important aspect of a simplification system is determining when an expression $\mathrm{X}$ is equal to another expression $\mathrm{Y}$, providing a mechanism for evaluating rules in the ruleset. This is fairly trivial when comparing single nodes, as one needs simply to check whether the nodes are identical. However, checking whether multi-node subexpressions/subtrees are equal is more difficult. Additionally, since one of our major goals is to provide simplification without degrading the performance of GP, this method needs to be fast.

Our goal is to allow for not only noticeably similar expressions (e.g. $(x+y+z)$ and $(z+x+y))$ to be identified as equivalent, but also

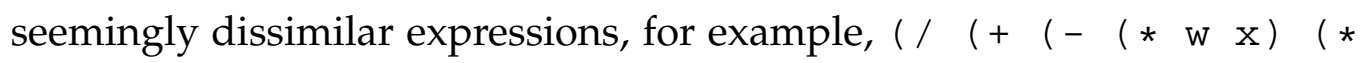
$\mathrm{x} y))(*(-\mathrm{w} y) \mathrm{y}))(-(\star \mathrm{x} x)(* \mathrm{y} y)))$ and $(/(-\mathrm{w} y)$ $(-\mathrm{x} y))$ as well.

In our approach, we use hashing to address the algebraic equivalence of two subtrees/subprograms. The hashing function is used to extend the algebraic system mentioned earlier to be capable of simplifying more 
expressions. In the 1970s and 1980s, [53] and [26] describe methods for achieving algebraic equivalence using hashing methods for algebraic expressions. In this approach, we use a variant of those methods to cope with all common terminals and functions in standard GP.

Note that by using the hashing technique to determine algebraic equivalence we add the risk of two non-equivalent subexpressions being determined as equal and one or both being discarded (depending on the simplification rule). By using a very large number of distinct hash values, the number of collisions can be kept minimal, and probabilistically minute. In this work, $p$ is used to denote the hashing order for the hash function (i.e. the total number of possible hash values).

It is important that the collection of hash values qualify as a finite field ([48]) and so $p$ should be a prime number. Note that any finite field with $p$ elements is isomorphic to $\mathbb{Z}_{p}[48]$ (the integers from 0 to $p$ ). In this work we have used a $p$ value of 1000077157 which is sufficiently large to reduce collisions and meets the prime number condition. Values within this field can also be manipulated using 64-bit integer arithmetic without overflow occurring (e.g. $1000077156 \times 1000077156<2^{64}$ ), which is important for implementation.

In the rest of this subsection, we describe how to estimate the algebraic equivalence for the terminals and operators in standard GP: feature terminals, constant terminals, the four arithmetic operators and the conditional operator.

\section{Feature Terminals}

In a GP system, a feature terminal represent inputs from the task environment, such as an image feature in object classification or a variable in symbolic regression. These feature terminals will thus have different values according to the fitness case being evaluated. The key property of these terminals is that a feature terminal always keeps the same value for a particular fitness case for all genetic programs during the evolutionary 
process. Thus, it does not matter what value we assign each feature terminal, as long as it is kept the same for each feature terminal throughout the evolutionary process. Accordingly, in this approach, the feature terminals are assigned random hash values at the beginning of the GP system run and remain unchanged for the entire duration of the evolutionary process. Specifically, we use (for each feature $n$ ):

$$
\operatorname{Hash}_{\left(\text {Feature }_{n}\right)}=\text { a random value in } \mathbb{Z}_{p}
$$

\section{Constant Terminals}

In a GP system, constants can be any numeric type: integers, rationals, floating point, etc. Therefore, the hash function needs to be designed to handle all these types. Integers and rationals can be easily handled in the following ways. For an integer $i \in \mathbb{Z}$, we can hash $i$ using equation 4.2.

$$
\forall i \in \mathbb{Z}, \operatorname{Hash}(i)=i \bmod p
$$

For processing a rational number $q \in \mathbb{Q}$, we first convert it into an arithmetic expression of two integers (equation 4.3). We will explain how to hash the arithmetic operators later in section 4.2.3.

$$
\begin{aligned}
\forall q \in \mathbb{Q}, q & =\frac{a}{b} \text { for some } a, b \in \mathbb{Z} \\
\therefore \operatorname{Hash}(q) & =\operatorname{Hash}\left(\frac{a}{b}\right)
\end{aligned}
$$

The most difficult of the common numerical types (excluding types such as imaginary numbers) are real valued numbers, represented in computers as floating point. [53] does not describe a solution to this in their paper. We resolve this problem by approximating the floating point with a rational number, thus converting it to a simple division of two integers.

Calculating accurate and irreducible (for $\frac{a}{b}, a$ and $b$ share no common factors) rationals can be very time consuming, so a quick and flexible approximation is used. The numerator is formed by multiplying the floating 
point by a predefined precision constant $(\delta)$ and truncating the leftover fractional part. Using the same precision constant as a denominator, a rational representation can be very quickly found. Another advantage is that $\delta$ can be easily tweaked to provide different levels of preserved precision.

$$
\operatorname{Hash}(c)=\frac{c \times \delta}{\delta} \bmod p=(c \times \delta) \times \frac{1}{\delta} \bmod p
$$

This approach (along with processing rationals in general) requires modular division, which one may not be familiar with.

Now, the division of two numbers $\frac{x}{y}$ is equivalent to the multiplication of the first number $(x)$ with the multiplicative inverse of the second number $(y)$ i.e. $x \times \frac{1}{y}$. Therefore, to perform division, one needs only to calculate the multiplicative inverse of $y$ and then multiply the result by $x$.

In processing constant terminals, $y$ is merely $\delta$. So the key point here is to find the integer equivalent of the inverse of $\delta \bmod p$. In this approach, this is done using the Extended Euclidean Algorithm [92,12]. For any two integers $a$ and $b$, there exists two integers $q, r$ such that $a=b \cdot q+r$. Commonly, $q$ is called the quotient and $r$ the remainder. Starting from step 0 , the algorithm additionally calculates an auxiliary number $x_{i}$ at each division step, where $x_{0}=0, x_{1}=1$ and for the other steps $x_{i}=$ $\left(x_{i-2}-x_{i-1} \cdot q_{i-2}\right) \bmod p$. At step $i$, the division is performed in the format of $a_{i}=q_{i} \cdot b_{i}+r_{i}$, where $a_{i}=b_{i-1}, b_{i}=r_{i-1}$ and $a_{0}=a, b_{0}=b$ at step 0 . The resulting $x$ will be the equivalence of the inverse of $a \bmod b$.

As an example, assuming that $\delta=10$, the constant to be hashed $c=0.6$ and the hash order $p=17$, then we have

$$
\operatorname{Hash}(0.6)=\frac{0.6 \times 10}{10} \bmod 17=6 \times \frac{1}{10} \bmod 17
$$

Now we use the extended Euclidean algorithm to find the integer equivalence of $\frac{1}{10}$ (the inverse of 10 in $\mathbb{Z}_{17}$ ). Here, $a_{0}=p=17, b_{0}=\delta=10$. Each step of the algorithm is shown below to calculate $x_{i}$, the value of $x$ at that step. 


$$
\begin{array}{ll}
\text { Step 0: } 17=1(10)+7 & x_{0}=0 \\
\text { Step 1: } 10=1(7)+3 & x_{1}=1 \\
\text { Step 2: } 7=2(3)+1 & x_{2}=\left(x_{0}-x_{1} \cdot q_{0}\right) \bmod p \\
& =(0-1 \cdot 1) \bmod 17=16 \\
\text { Step 3: } 3=3(1)+0 & x_{3}=\left(x_{1}-x_{2} \cdot q_{1}\right) \bmod p \\
& =(1-16 \cdot 1) \bmod 17=2 \\
\text { Step 4: } & x_{4}=\left(x_{2}-x_{3} \cdot q_{2}\right) \bmod p \\
& =(16-2 \cdot 2) \bmod 17=12
\end{array}
$$

The last value for $x$ is 12 , meaning that the integer equivalence of the inverse of $10\left(\frac{1}{10}\right.$ in $\left.\mathbb{Z}_{17}\right)$ is 12 . A quick check shows that $12 \times 10 \bmod 17=$ $120 \bmod 17=1$, so this is indeed correct. Substituting this value 12 in for $\frac{1}{10}$ in equation 4.5 , we have

$$
6 \times \frac{1}{10} \bmod 17=6 \times 12 \bmod 17=72 \bmod 17=4
$$

So the constant 0.6 hashes to the value 4 in this example.

\section{The Arithmetic Operators}

Because the hashing method takes place in a finite field, all of the standard arithmetic methods are easily handled using modulo arithmetic. Hashing of these operators is equivalent to evaluating them within the field:

$$
\begin{aligned}
& \text { Hash }(A+B)=(A+B) \bmod p \\
& \text { Hash }(A-B)=(A-B) \bmod p \\
& \text { Hash }(A \times B)=(A \times B) \bmod p \\
& \text { Hash }(A \div B)=(A \div B) \bmod p
\end{aligned}
$$

where the division hashing follows the process of the extended Euclidean algorithm discussed above, using Hash(B) instead of $\delta$ and multiplying the result by Hash(A). 


\section{The if $<0$ operator}

The if $<0$ conditional operator is a more difficult case, as it is not an arithmetic function and so cannot simply be converted to a modulo arithmetic equivalent. Additionally, it consists of three parameters (instead of the usual two): a condition, a true branch and a false branch. All the three parameters must be considered when hashing this operator as well as the order in which they appear. We need to attempt to avoid devising a method in which two obviously different if $<0$ statements are hashed to the same value. Suppose we were to use $\operatorname{Hash}($ if $<0(A, B, C))=A+B-C$. This would take into account $C$ being in a different position than A or B. Unfortunately, because of the commutativity of + , hashing if $<0$ A B C would be equivalent to hashing if $<0 B$ A $C$. It is therefore important that we use multiplication/division as well as addition/subtraction in our if $<0$ hashing method. One such method is the following:

$$
\operatorname{Hash}(\mathrm{if}<0(A, B, C))=\left(\frac{A}{B}+C\right) \bmod p
$$

Work in [102] highlights a situation in which two seemingly different if $<0$ statements are actually equivalent, and argues that a good formulation for handling if $<0$ would handle this situation successfully. We briefly look at the possibilities of doing that here:

$$
\begin{array}{lllllll}
i f<0 & A & (i f<0 & \text { B } & \text { C } & \text { D }) & \text { D }) \\
i f<0 & \text { B } & (i f<0 & \text { A } & \text { C } & \text { D }) & \text { D })
\end{array}
$$

Figure 4.1: Two equivalent statements given in [102]

These statements both essentially say: "if A and B are less than zero, then $C$, otherwise $D^{\prime \prime}$. Keeping this in mind, we can use the following approach to handle this situation:

$$
\operatorname{Hash}(\mathrm{if}<0(A, B, C))=\left(\frac{A}{C}+B\right) \bmod p
$$


which again uses division and addition to take into account the position of the three parameters. For the two statements in figure 4.1, we get the following:

$$
\begin{aligned}
& \text { if }<0 \text { A }(\text { if }<0 \quad \text { B C D }) \quad \text { D })=\left(\frac{A}{D}+\frac{B}{D}+C\right) \bmod p \\
& \text { if }<0 \text { B }(\text { if }<0 \text { A } \quad \text { C D }) \quad \text { D })=\left(\frac{B}{D}+\frac{A}{D}+C\right) \bmod p
\end{aligned}
$$

Which both hash to the same value successfully. However, consider the next two statements:

$$
\begin{aligned}
& \text { if }<0 \text { A }(i f<0 \text { B D C) D) } \\
& \text { if }<0 \text { B D }(\text { if }<0 \text { A } C \text { D) })
\end{aligned}
$$

Figure 4.2: Another pair of equivalent statements

These two statements essentially say: "if A is less than zero and B is greater than or equal to zero, then $C$, otherwise $D^{\prime \prime}$. The previous formulation for hashing if $<0$ cannot successfully equate these two statements.

It would be very difficult (if not impossible) to design a modular arithmetic function that could handle all the possible equivalences that exist when using the if<0 statement. The most important aspect is that we avoid the most common, basic forms of collisions (e.g. rearranged order of children). Hence we use the following formulation for hashing if $<0$ :

$$
\operatorname{Hash}(\text { if }<0(A, B, C))=\left(\frac{A}{B}+C\right) \bmod p
$$

\section{Operator Closure}

All of the functions supported are closed, meaning that for any of the functions $\diamond \in\{+,-, \times, \div$, if $<0\},(\operatorname{Hash}(A) \diamond \operatorname{Hash}(B)) \bmod p=\operatorname{Hash}(A \diamond B)$ in $\mathbb{Z}_{p}$. More specifically:

$$
\operatorname{Hash}(A+B)=(\operatorname{Hash}(A)+\operatorname{Hash}(B)) \bmod p
$$




$$
\begin{aligned}
H a s h(A-B) & =(\operatorname{Hash}(A)-\operatorname{Hash}(B)) \bmod p \\
H \operatorname{aash}(A \times B) & =(\operatorname{Hash}(A) \times \operatorname{Hash}(B)) \bmod p \\
H \operatorname{ash}(A \div B) & =(\operatorname{Hash}(A) \div \operatorname{Hash}(B)) \bmod p \\
H a \operatorname{tash}(\text { if }<0(A \diamond B, C, D)) & =\left(\frac{\operatorname{Hash}(A \diamond B)}{\operatorname{Hash}(C)}+\operatorname{Hash}(D)\right) \bmod p
\end{aligned}
$$

This is important to the overall performance of the simplification system. It means we can store the calculated hash values inside each node as they are processed. In order to calculate the hash of a later (parent) node, we only need to fetch the stored hash values of its children. There is no need to recalculate the hash value of the entire subtree, and hence the hash value for every node need only be calculated once.

\subsubsection{An Example}

Now, we use an example to show the simplification process for a given genetic program. The example genetic program $(-(--0.2-0.5) \quad($ if $<0$ $(\%(+f 0 f 1)(+f 1 f 0)) 0.8(-f 0 f 0)))$ can be represented in the tree shown in figure 4.3. Note that we use $\%$ to denote the protected division operator.

Assume that the hashing order is $17, \mathrm{f} 0$ and $\mathrm{f} 1$ are "randomly" assigned the values 3 and 5 respectively. Also, for presentation convenience, Table 4.2 reiterates the rules in the ruleset that are specifically used in this example.

The algorithm traverses the program tree in a "bottom-up" fashion using a postfix traversal. This means that the algorithm processes the program nodes in the order depicted in figure 4.4.

The first node inspected by the algorithm is " -0.2 ", followed by " -0.5 ". As no simplification rule exists in the ruleset that governs single nodes, these nodes (and indeed the entire bottom layer of nodes) are left unchanged. Next, the algorithm moves to the parent node of " -0.2 " and 


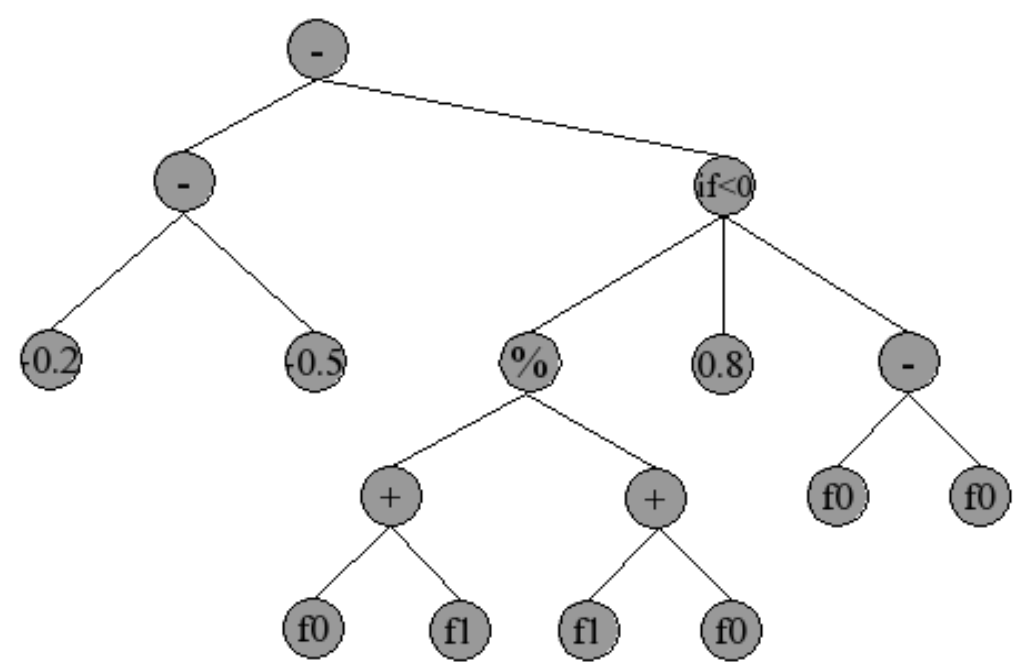

Figure 4.3: The original program tree.

Table 4.2: Simplification rules used in this example.

\begin{tabular}{|lll|}
\hline Precondition & & Effective Result \\
\hline$(1)(-\mathrm{a}$ b $)$ & $\rightarrow \mathrm{C}, \mathrm{C}=\mathrm{a}-\mathrm{b}$ \\
$(2)(\% \mathrm{~A} A)$ & $\rightarrow 1$ \\
$(3)(-\mathrm{A}$ A $)$ & $\rightarrow 0$ \\
$(4)($ if $<0$ a B C $)$ & $\rightarrow \mathrm{C}($ if $\mathrm{a} \geq 0)$ \\
\hline
\end{tabular}

" -0.5 ", which is "-". The subtree formed by this node and its children $(--0.2-0.5)$ matches the precondition for rule $(1)(-a b)$. The system applies this rule, replacing the subtree with the rule's effective result: "0.3".

Now, the subtrees (+ f0 $f 1$ ) and ( $f 1$ f0) do not match the preconditions for any of the rules, so are left unchanged. Note however, that they both have the same algebraic equivalence hash value (shown in figure 4.5). Therefore, when node 10 ("응") is inspected, the subtree $(\%$ $f 0 \mathrm{fl})(+\mathrm{f} 1 \mathrm{f} 0)$ ) does indeed match the precondition for rule (2) (\% A A). The entire subtree is replaced using the rule to a single node 1 . Sim- 


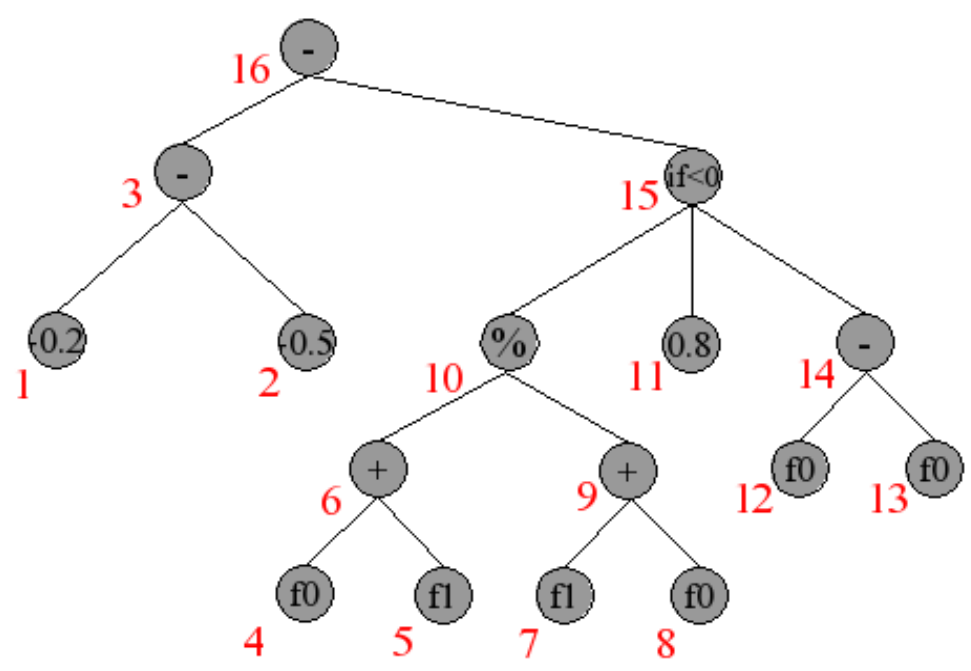

Figure 4.4: Bottom-up traversal order (shown by integer values).

ilarly, the subtree ( $f 0 \mathrm{fO}$ ) matches rule (3) (- A A) and is replaced by the single node 0 when the algorithm processes " - ".

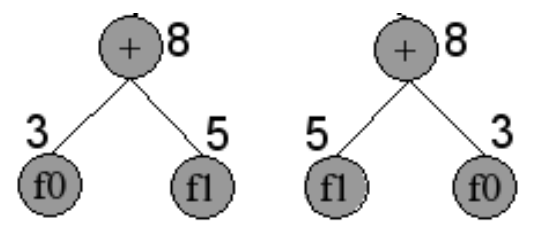

Figure 4.5: Hashing of two subtrees with same value (shown by integer values).

Figure 4.6 shows the tree after processing nodes 1 through 14.

At this stage, the program is already reduced to 6 nodes in size, and there are still two nodes left to be processed. Inspecting the if $<0$ node, the algorithm matches it with rule (4) (if $<0$ C A B), as the first parameter of the if $<0$ operator is a constant. In this case, the constant is 1 , which will obviously never be less than 0 . The system then, following the rule, replaces this subtree with its third parameter, which is 0 .

Lastly the root node is processed, which again matches rule (1) $(-$ a b). Applying it yields the final result, a single numerical constant node 


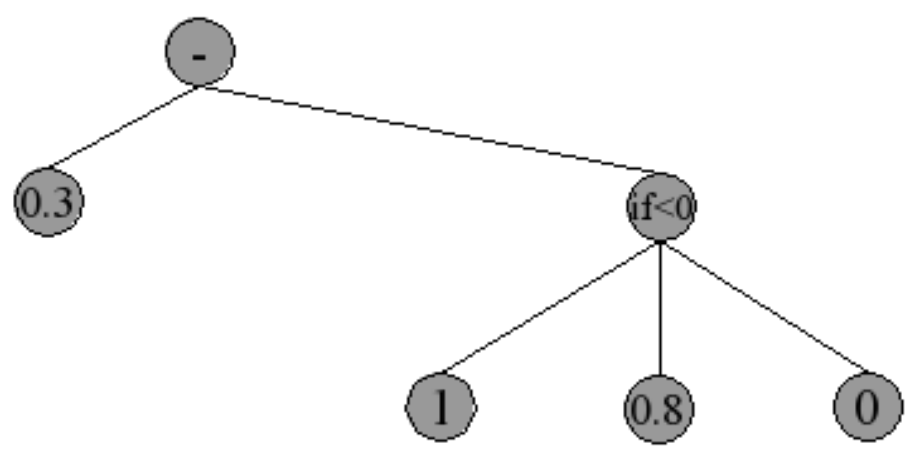

Figure 4.6: The program after partial processing.

“0.3" (figure 4.7).

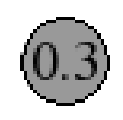

Figure 4.7: The final program, a single node.

\subsubsection{Summary of the Simplification Algorithm}

The simplification algorithm simplifies a given program in the following way:

- Traverse the program tree in a bottom-up fashion using a postfix mode.

- For the terminal nodes, calculate the equivalence hash values.

- For each non-terminal node

- Calculate the equivalence hash value of the node, directly using the hash values of its child nodes.

- Iterate through the set of rules. If the node (and its surrounding nodes) match the precondition of a rule, apply that rule to simplify the subtree associated with the node. 
- Once all nodes have been processed, output the final, simplified program tree.

This algorithm is invoked on a number of programs in the GP system, replacing the original programs with their simplified counterparts.

\subsection{Experimental Setup}

Here we used six of the GP tasks outlined in chapter 3. These are the three symbolic regression tasks (regression ${ }_{1}$, regression $_{2}$ and regression ${ }_{3}$ ), an image classification task (shapes) and the two medical classification tasks (breast cancer Wisconsin and SPECT).

\subsection{Results and Discussion}

Tables 4.3 and 4.4 displays the means and standard deviations of various measures taken from 50 individual GP runs for each of the regression and classification tasks. These measured aspects are the number of generations completed at the end of GP evolution (Gens), the training fitness of the "best" program in the final generation (Best Fitness), the average size (in number of nodes) of programs found in all generations (Avg. Prog Size) and for the classification tasks: the testing fitness of the "best" program in the final generation (Best Test Fitness). Note that the "best" programs evaluated in order to get the training and testing fitnesses are the same program, as the measure for "best" program for a particular GP run is only governed by the fitness function, which works on training fitness alone.

\section{Program Size}

As can be seen from the sixth column of tables 4.3 and 4.4, for all tasks using simplification results in a drop in the average program size over the standard GP. This is perfectly in line with what we want to achieve, as the 
Table 4.3: Results from the three regression GP tasks: Regression ${ }_{1}$, Regression $_{2}$, Regression . $_{\text {. }}$

\begin{tabular}{|l|c|c|c|c|c|c|}
\hline Task & Frequency & Gens & Best Fitness & Time(s) & Avg. Prog Size & Best Test Fitness \\
\hline \multirow{6}{*}{$\operatorname{Reg}_{1}$} & Without & $47.9 \pm 5.88$ & $0.1524 \pm 0.4332$ & $1.89 \pm 0.42$ & $40.95 \pm 14.50$ & N/A \\
& Every 1 & $48.58 \pm 4.88$ & $0.0650 \pm 0.1619$ & $2.01 \pm 0.32$ & $26.83 \pm 12.58$ & N/A \\
& Every 2 & $48.64 \pm 4.87$ & $0.0762 \pm 0.2574$ & $1.67 \pm 0.29$ & $27.32 \pm 12.68$ & N/A \\
& Every 3 & $49.02 \pm 4.10$ & $0.0629 \pm 0.1414$ & $1.62 \pm 0.25$ & $28.16 \pm 12.75$ & N/A \\
& Every 4 & $48.84 \pm 4.04$ & $0.0850 \pm 0.2158$ & $1.56 \pm 0.24$ & $28.51 \pm 12.69$ & N/A \\
& Every 5 & $49.34 \pm 3.28$ & $0.1252 \pm 0.3024$ & $1.58 \pm 0.23$ & $29.45 \pm 13.37$ & N/A \\
\hline \multirow{6}{*}{$\operatorname{Reg}_{2}$} & Without & $50 \pm 0$ & $1186.13 \pm 1064.92$ & $2.32 \pm 0.45$ & $49.78 \pm 16.86$ & N/A \\
& Every 1 & $50 \pm 0$ & $1309.3 \pm 1153.47$ & $2.65 \pm 0.40$ & $35.13 \pm 12.73$ & N/A \\
& Every 2 & $50 \pm 0$ & $1273.05 \pm 1013.44$ & $2.18 \pm 0.30$ & $35.21 \pm 12.60$ & N/A \\
& Every 3 & $50 \pm 0$ & $1459.28 \pm 1267.41$ & $2.13 \pm 0.38$ & $37.07 \pm 13.74$ & N/A \\
& Every 4 & $50 \pm 0$ & $1070.29 \pm 1156.76$ & $1.91 \pm 0.28$ & $34.73 \pm 12.76$ & N/A \\
& Every 5 & $50 \pm 0$ & $1148.97 \pm 887.056$ & $1.95 \pm 0.31$ & $36.96 \pm 13.66$ & N/A \\
\hline \multirow{6}{*}{$\operatorname{Reg}_{3}$} & Without & $50 \pm 0$ & $140.071 \pm 362.445$ & $2.38 \pm 0.41$ & $50.83 \pm 16.11$ & N/A \\
& Every 1 & $50 \pm 0$ & $159.576 \pm 296.105$ & $2.90 \pm 0.44$ & $37.23 \pm 12.87$ & N/A \\
& Every 2 & $50 \pm 0$ & $103.836 \pm 91.422$ & $2.34 \pm 0.27$ & $37.44 \pm 12.22$ & N/A \\
& Every 3 & $50 \pm 0$ & $89.817 \pm 88.637$ & $2.22 \pm 0.33$ & $38.30 \pm 13.04$ & N/A \\
& Every 4 & $50 \pm 0$ & $154.999 \pm 319.870$ & $2.12 \pm 0.28$ & $38.66 \pm 12.71$ & N/A \\
& Every 5 & $50 \pm 0$ & $96.822 \pm 100.048$ & $2.07 \pm 0.28$ & $38.74 \pm 12.65$ & N/A \\
\hline
\end{tabular}


Table 4.4: Results from the four classification GP tasks: Shapes, Breast Cancer Wisconsin and SPECT.

Cancer Wisconsin and SPECT.
\begin{tabular}{|c|c|c|c|c|c|c|}
\hline Task & Frequency & Gens & Best Fitness & Time(s) & Avg. Prog Size & Best Test Fitness \\
\hline \multirow{5}{*}{ Shapes } & Without & $50 \pm 0$ & $0.957 \pm 0.0119$ & $4.62 \pm 0.93$ & $45.19 \pm 16.15$ & $0.950 \pm 0.0144$ \\
& Every 1 & $50 \pm 0$ & $0.957 \pm 0.0122$ & $4.65 \pm 1.04$ & $34.39 \pm 14.24$ & $0.952 \pm 0.0155$ \\
& Every 2 & $50 \pm 0$ & $0.960 \pm 0.0097$ & $4.09 \pm 0.83$ & $34.01 \pm 14.40$ & $0.953 \pm 0.0122$ \\
& Every 3 & $50 \pm 0$ & $0.960 \pm 0.0170$ & $3.94 \pm 0.82$ & $34.12 \pm 14.43$ & $0.953 \pm 0.0185$ \\
& Every 4 & $50 \pm 0$ & $0.959 \pm 0.0117$ & $3.92 \pm 0.86$ & $34.52 \pm 14.64$ & $0.951 \pm 0.0127$ \\
& Every 5 & $50 \pm 0$ & $0.961 \pm 0.0113$ & $3.91 \pm 0.71$ & $35.12 \pm 13.98$ & $0.954 \pm 0.0119$ \\
\hline \multirow{5}{*}{ BCW } & Without & $50 \pm 0$ & $0.949 \pm 0.0136$ & $2.22 \pm 0.46$ & $37.99 \pm 14.58$ & $0.975 \pm 0.0135$ \\
& Every 1 & $50 \pm 0$ & $0.951 \pm 0.0092$ & $2.55 \pm 0.50$ & $28.38 \pm 13.65$ & $0.971 \pm 0.0181$ \\
& Every 2 & $50 \pm 0$ & $0.949 \pm 0.0126$ & $2.03 \pm 0.38$ & $27.07 \pm 13.02$ & $0.975 \pm 0.0116$ \\
& Every 3 & $50 \pm 0$ & $0.951 \pm 0.0117$ & $1.98 \pm 0.39$ & $28.02 \pm 13.63$ & $0.978 \pm 0.0105$ \\
& Every 4 & $50 \pm 0$ & $0.950 \pm 0.0105$ & $1.92 \pm 0.36$ & $28.49 \pm 13.35$ & $0.978 \pm 0.0098$ \\
& Every 5 & $50 \pm 0$ & $0.951 \pm 0.0105$ & $1.89 \pm 0.36$ & $28.60 \pm 13.70$ & $0.975 \pm 0.0127$ \\
\hline \multirow{5}{*}{ SPECT } & Without & $50 \pm 0$ & $0.761 \pm 0.0333$ & $1.54 \pm 0.19$ & $38.47 \pm 13.04$ & $0.795 \pm 0.0279$ \\
& Every 1 & $50 \pm 0$ & $0.789 \pm 0.0429$ & $2.14 \pm 0.31$ & $29.67 \pm 13.46$ & $0.795 \pm 0.0287$ \\
& Every 2 & $50 \pm 0$ & $0.784 \pm 0.0448$ & $1.70 \pm 0.25$ & $30.11 \pm 14.32$ & $0.797 \pm 0.0236$ \\
& Every 3 & $50 \pm 0$ & $0.778 \pm 0.0397$ & $1.55 \pm 0.25$ & $29.86 \pm 14.94$ & $0.791 \pm 0.0267$ \\
& Every 4 & $50 \pm 0$ & $0.780 \pm 0.0422$ & $1.51 \pm 0.24$ & $30.68 \pm 15.42$ & $0.792 \pm 0.0279$ \\
& Every 5 & $50 \pm 0$ & $0.771 \pm 0.0373$ & $1.45 \pm 0.22$ & $30.67 \pm 15.25$ & $0.791 \pm 0.0292$ \\
\hline
\end{tabular}


purpose of simplification is to reduce the size of programs and hence save computer resources. We found that in all the tasks, and at all the simplification frequencies, we get a significant drop in average program size to a $95 \%$ confidence interval (using statistical z-testing). This shows that using the simplification method produces statistically significant reductions in the average program sizes. As a large amount of the memory used by a GP system is used by the population of programs, this reduction in average size corresponds to a significant decrease in memory usage.

To do a further analysis, we also present the average size of genetic programs at every generation for the six tasks in figures 4.8 and 4.9. We found that performing simplification at every generation produced the smallest programs out of all the systems. While performing simplification at lower frequencies generally results in a higher average program size than performing at every generation, this increase in size is very small, suggesting that simplification does not need to be performed at every generation in order to be effective.

\section{CPU Time}

Another point of interest is whether this reduction in program size can translate into a reduction in the amount of time taken by GP to find a solution. Using simplification generally results in smaller programs, which should be processed faster when performing the other parts of GP (e.g. fitness evaluation). From column five of the results tables 4.3 and 4.4, it is clear that using simplification in every generation leads to an increase in the amount of time GP takes to find solutions. This indicates that the overhead from using simplification at every generation outweighs the benefits gained from the smaller programs. However, in almost all cases (only the SPECT dataset with simplification every 2 generations is the exception) when simplifying every two to five generations, we get a significant reduction in the CPU time taken. This suggests that it is always possible to find a good frequency for simplification that can significantly reduce 


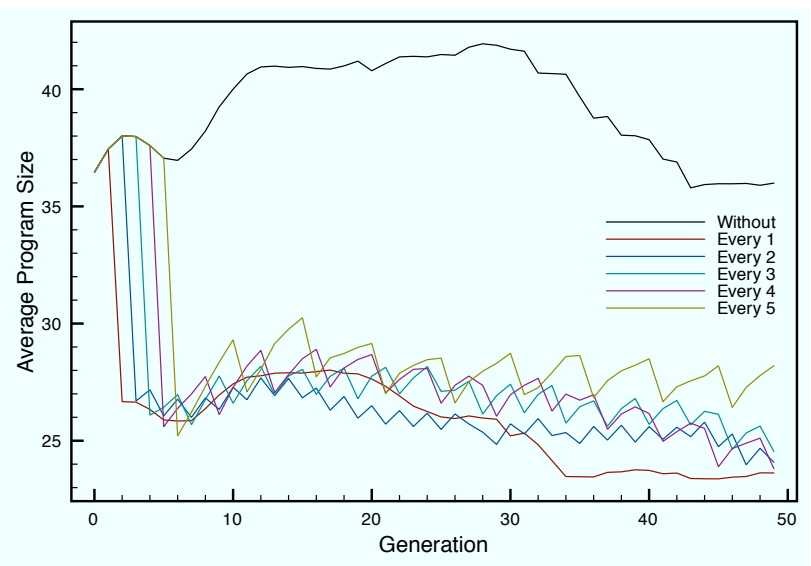

Regression $_{1}$

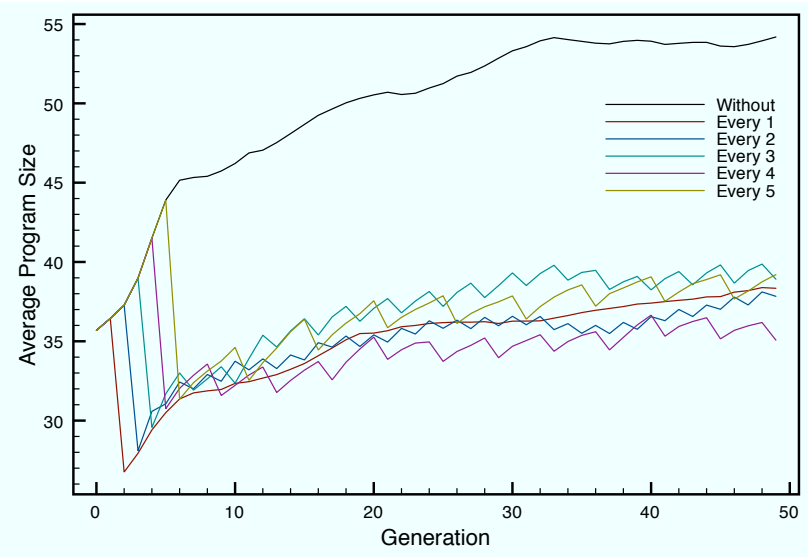

Regression $_{2}$

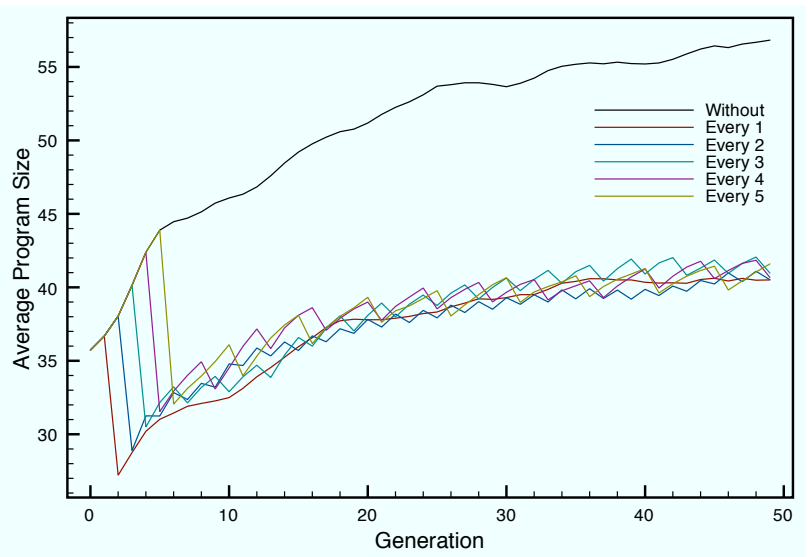

Regression $_{3}$

Figure 4.8: Average Program Size per Generation, for Regression Tasks. 


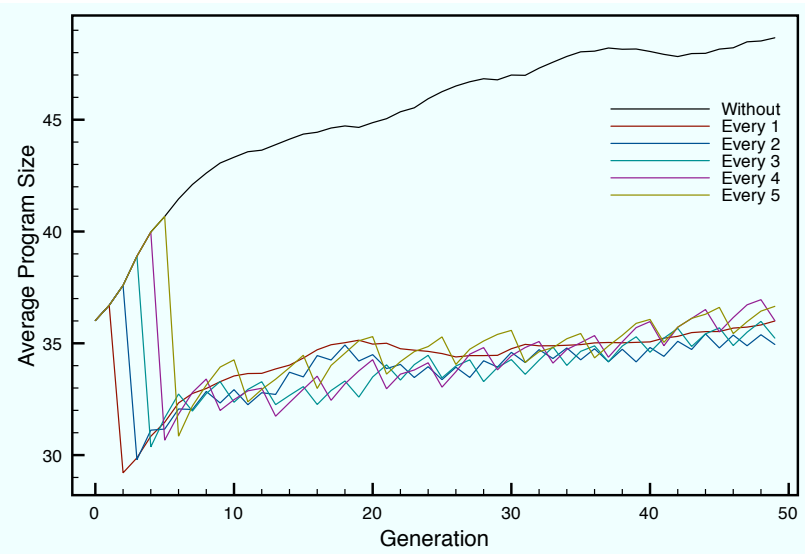

Shapes

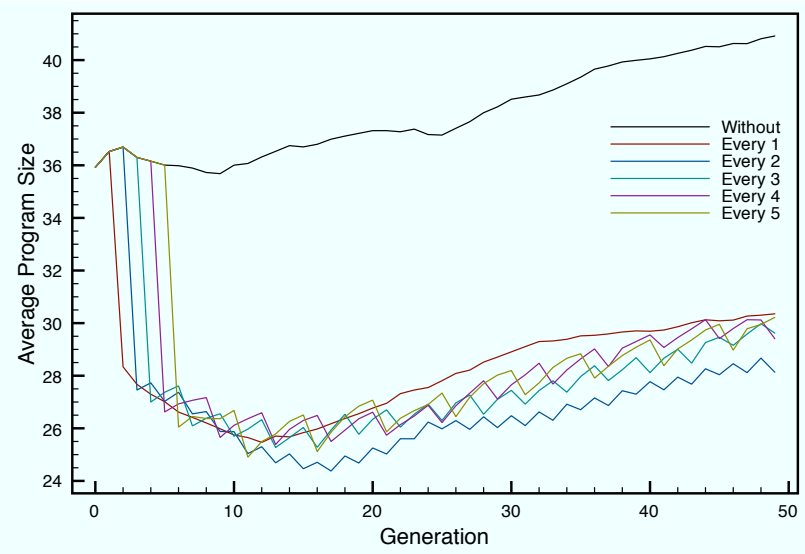

$\mathrm{BCW}$

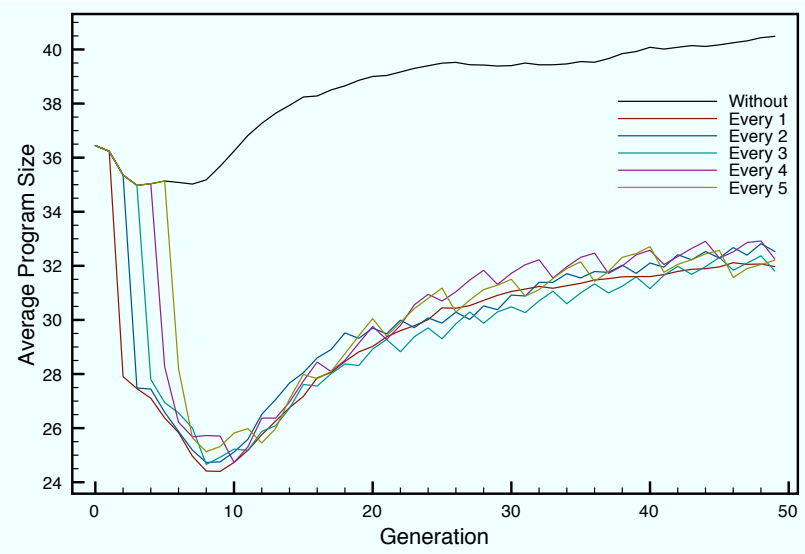

SPECT

Figure 4.9: Average Program Size per Generation, for Classification Tasks. 
program size and shorten CPU time.

Using different frequencies leads to different CPU times, and there does not seem to be a reliable way to set the best frequency in the simplification approach, which would usually require an empirical search. However, if such a search can arrive in a good frequency in the simplification that can require significantly shorter time, produce significant smaller program sizes, result in comparable or even better accuracy (effectiveness) than the standard GP, such a small price to pay. Based on our experiments, it seems that a frequency in the range of 3-5 may serve as a good starting point.

\section{Effectiveness}

Column four of results tables 4.3 and 4.4 shows the average fitness of evolved programs on the training dataset, while column seven shows the fitness of evolved programs on the testing dataset. Using statistical ztesting, we found that in almost all cases there was no statistical difference in the performances of the "best" programs from systems using simplification and the standard GP (to a 95\% confidence interval).

These results suggest that using our simplification method has no significant impact on the effectiveness of the programs produced by the GP evolution. In some cases (such as Regression and Regression $_{3}$ at every 3 or 4 generations, the Shapes dataset at every 5 generations and the SPECT dataset for all frequencies) the GP system using simplification was able to obtain significantly better training fitness than the standard GP. This is important for our method, as although it is desirable to reduce the program sizes, doing so in exchange for system effectiveness is best to be avoided if possible. Our simplification method appears to be able to fulfill its aims of reducing program size in the GP populations without deteriorating the system effectiveness, and may even improve the effectiveness in some cases. In the past, it has been thought that simplification would lead to a deterioration in program effectiveness as building blocks may be 
disrupted by either the simplification itself, or through being exposed to crossover. Our results suggest that no loss in effectiveness takes place. We hypothesise that even though building blocks may be disrupted early on in the GP evolution, using simplification may actually offset this by introducing new building blocks into the GP populations. We will investigate this hypothesis further in the next chapter.

\subsection{Chapter Summary}

In this chapter, (in line with our first goal) we presented our own algorithm for performing algebraic simplification of GP programs during the evolutionary process. This algorithm uses simple algebraic rules and an equivalence hashing technique to determine the equivalence of GP program subtrees. We integrated this approach into a standard GP system and enabled it to simplify all programs within certain GP populations. Unlike some past methods, our program simplification method was applied in an online manner, meaning that simplification was applied to the populations during the evolution. A frequency parameter was used to determine in which generation(s) the GP population would be simplified. This allowed an element of control of how aggressively we would apply simplification. GP systems using simplification were used to perform seven different tasks (three symbolic regression, two image classification and two medical classification) and then compared to a standard GP system, paying particular attention to both the efficiency of the systems and the effectiveness of the system in producing quality solutions.

Our second goal was to determine whether using this simplification system can significantly reduce the size of programs in the GP populations. The results gathered from these experiments suggest that using simplification will lead to a reduction in the size of programs held in the GP populations, and can reduce the CPU time that GP takes to evolve solutions. While using simplification at every generation produced the largest 
reduction in program size in most tasks, it also led to an increase in the time used by GP to find a solution. A simplification frequency of 3 to 5 appears to be a good starting point when applying simplification to a new GP task.

In line with our third goal, simplification was able to reduce the program size without resulting in decreased effectiveness of GP in finding solution programs. This was a very important result, as it shows that simplification is a viable technique for combating the program bloat problem without sacrificing any of GP problem solving power.

These results in this chapter have shown us that our simplification method can result in smaller programs without harming the effectiveness of the GP system. However, it is still of interest to us to investigate how the use of simplification affects the "building-blocks" within the GP populations, particularly whether simplification disrupts and/or creates new building blocks. We develop an approach to performing this analysis in the next chapter. 
74 CHAPTER 4. ALGEBRAIC SIMPLIFICATION OF GP PROGRAMS 


\section{Chapter 5}

\section{Effects of Simplification on Simple Building-Blocks}

\subsection{Introduction}

It has often been thought that the redundancies that make up a large part of code bloat may protect programs from the destructive effects of GP crossover $[59,90,89]$ by reducing the chances of crossover/mutation points being selected within highly fit portions of a program, and that genetic systems are under evolutionary pressure to create these redundancies in order to increase the "robustness" of fit solutions [103]. Additionally, redundancies may contain valuable genetic materials that can be recombined in future generations to form better solutions [59]. As simplification removes these redundancies, they may also be destroying these genetic materials, reducing diversity and hindering the GP system's ability to find a good solution. We hypothesised in chapter 4 (page 71) that the simplification process may destroy potential building blocks early on, but can also generate new building blocks at later stages of the GP evolution, leading to no effect overall in the GP system's effectiveness. However, these hypotheses have not yet been seriously examined through experimentation. Further investigation on this topic is necessary to our understanding of simplifica- 
tion in GP.

\subsubsection{Chapter Goals}

This chapter aims to investigate and analyse the effects on GP building blocks, of using online simplification during the evolutionary process. We will achieve this by tracing simple building blocks throughout a number of individual GP runs both with and without simplification, and then examining the behaviour of the building blocks through these runs. More specifically, we will investigate the following issues:

- how building-blocks within GP be represented in a simple manner which can still hold significant meaning;

- how we can visualise and interpret these building-blocks as they are tracked through the GP evolution;

- investigate whether utilising a simplification component in a GP system disrupts potential building blocks in the GP population;

- determine whether simplification creates new building blocks in a GP population with which to form better solutions; and

- determine whether the creation of new building blocks can overcome the negative effects of building blocks being disrupted, and result in more accurate GP solutions.

\subsection{Numerical-Nodes as Simple Building Blocks}

For this chapter, we need to accurately track building blocks throughout a GP run in order to observe their behaviour and how they are affected by simplification. For this purpose, we narrow our focus to the simplest form of subtree: Single numerical terminal nodes (which we term simply as 
numerical-nodes). These are single nodes present in a program which are usually represented by a single floating point number. They are often used in GP tasks to contribute additional numerical constants toward a GP solution, and may be considered as parameter values of a mathematical model described by the rest of the program tree. In the standard GP, these are usually regarded as "constant" terminals. However, in GP systems utilising simplification, these terminals are no longer constant as they can be altered by the simplification process. These nodes easily serve as "tracers", which can be individually tracked during a GP run so that their behaviour can be monitored. By considering only single-nodes, any additional complexity in analysis that can be introduced by crossover taking place within a tracked building block is removed.

One may argue that purely focusing on numerical-nodes is a gross simplification of GP building blocks. However, numerical-nodes do exhibit the basic characteristics of building blocks. Numerical-nodes may be combined with other nodes to build larger subtrees that contribute to a more accurate solution. Like larger GP subtrees, numerical-nodes can either contribute towards a solution or merely act as noise, guiding the system away from the solution. Most importantly, they can be combined or removed by the simplification component used in a GP system and as such allows us to analyse any possible disruption. With regards to what a building block is conceptually (small components that can be formed into larger, more fit components), numerical-nodes can be easily regarded as building blocks. Previous work performed in [14] focused on testing numericalnodes as possible building blocks using a symbolic regression problem. Their work supports the notion that numerical-nodes, despite their simplicity, do exhibit characteristics of building blocks and are "significant to understanding the dynamics of GP for more than just their structural (single-node) aspect". We believe that the use of numerical-nodes provides a good "first step" into analysing the influence simplification has on GP building blocks. We will consider and analyse larger and more com- 
plex forms of building blocks in the next chapter.

\subsubsection{Visualisation and Interpretation of Numerical-Nodes}

In order to successfully track and subsequently interpret the movement of these numerical-node building blocks, it is highly desirable to be able to visually monitor these behaviours. Here we describe our developed method for visualising and analysing numerical-node building blocks.

During a GP evolutionary run we process every program in each generation. We record the values of each numerical-node as well as which generation they occur in, resulting in a list of (value, generation) pairs. These pairs are used to form scatterplots for each GP run, using the 100 generations as the $x$-axis and the numerical-node values as the y-axis. For each pair, we mark the corresponding position on the scatterplot with a ' + ' symbol. Using these plots, we can see which numerical-node values are present in the GP population for each generation.

Ordinarily, any two nodes which have identical values and occur in the same generation would only appear as a single ' + ' symbol (since the plot actually shows two ' + ' symbols directly overlapping). In order to make all nodes visible, we add a small amount of random noise to the recorded values before they are plotted. Figure 5.1(a) shows an example section of a typical scatterplot without noise, while figure 5.1(b) shows the same section with the added noise. The benefit from doing this is that now we can differentiate between highly populated (where many nodes have the same value) and lowly populated numerical-nodes. Highly populated numerical-node values will show up as a more dense portion of the plot, while lowly populated values will appear more separated and sparse.

Being able to identify highly populated regions allows us to determine which numerical-nodes in the GP system are surviving and propagating through the population and which are being removed. By examining which numerical-nodes are prevalent in standard GP runs and compared 
those with GP runs which use simplification, we can determine how simplification affects these numerical-nodes.

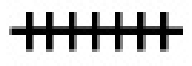

(a)

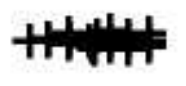

(b)

Figure 5.1: Examples of scatterplots (a) without noise and (b) with noise added

Figure 5.2 displays the scatterplot for a single GP run. Note that at generation 0 all of the numerical-node values are evenly distributed. This is expected, as the GP system randomly generates these values from a uniform distribution. Fairly quickly though, numerical-node values become concentrated to a small number of intervals, forming dense "bands" (such as the one marked by B). Similarly there are less dense regions (such as the one marked by A). These less dense regions show the numerical-node values which are undesirable to the GP system and therefore have been largely discarded. Because of genetic mutation (in which new numericalnodes may be randomly created), these sparse regions are almost never completely empty. This shift in the distribution of numerical-nodes shows that GP quickly discards all the non-useful numerical-nodes and propagates the few which are useful in performing the given task.

\subsubsection{Experimental Setup}

For experimentation, we used two symbolic regression tasks. The first task

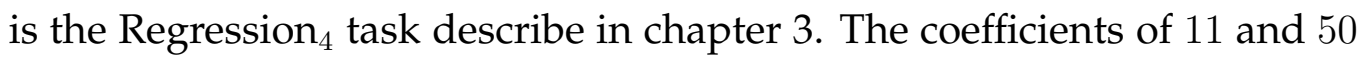
were chosen because they are fairly distant from each other and are not multiples of each other. This means that at least two different combinations of the numerical-nodes are needed to correctly regress this function. 


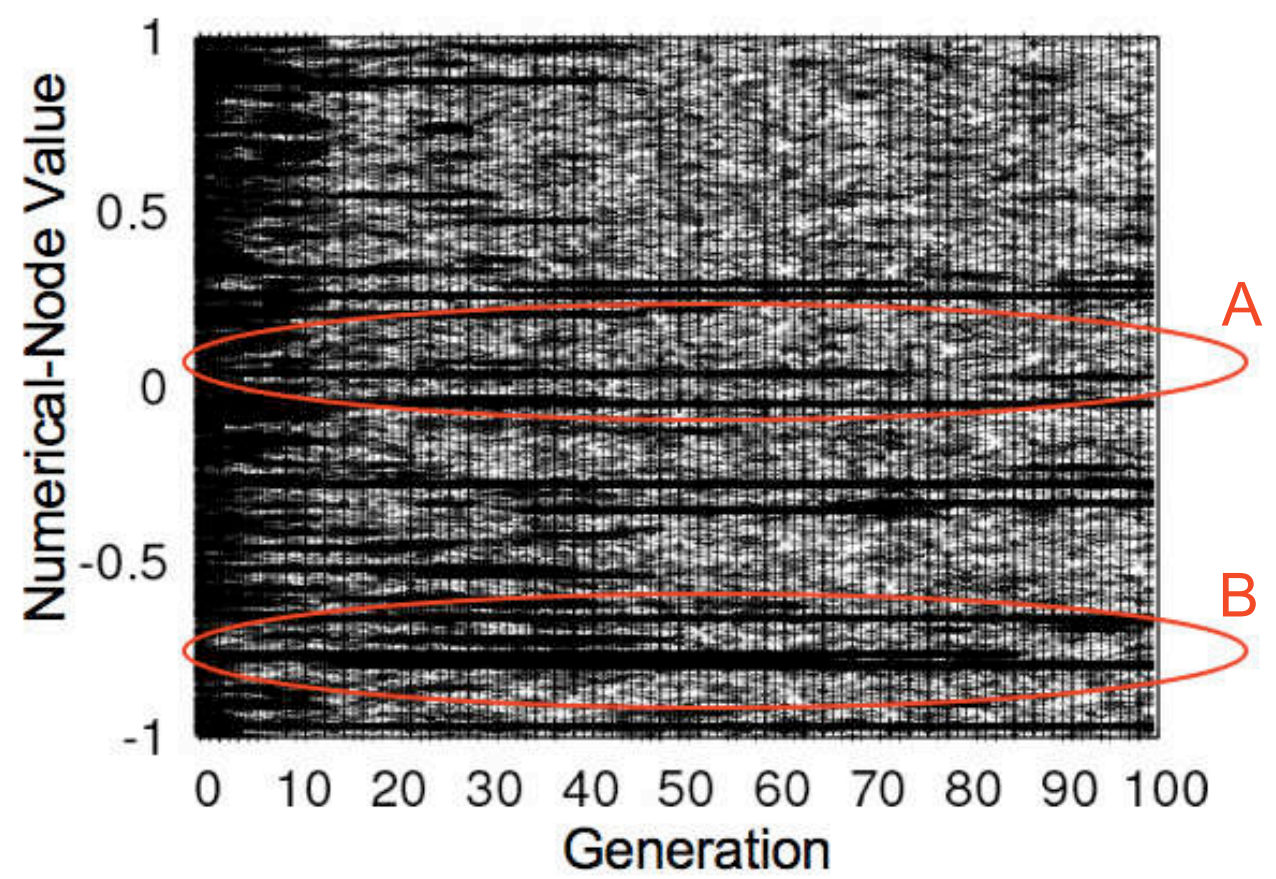

Figure 5.2: Numerical-Node Building Block Chart Example

A linear (single variable) equation was selected to place emphasis almost entirely on the numerical constants, so that the GP system would need to process numerical-nodes more often to form better solutions. The second task is the Regression 2 problem (also in chapter 3). This was chosen as it provides a more difficult problem than the first regression task, and has many different ways to build a "correct" solution.

Each numerical-node is generated during the initial program population generation, and is chosen from a range of $[-1.0,1.0]$. The use of a small range (in the context of the symbolic regression problems being used) is intentional, in order to make it clearer whether simplification is able to create new numerical-nodes. Also, the use of such an "unsuitable" initial range is able to increase the difficulty of the symbolic regression problem [15] particularly in the case of Regression 4 .

The settings used in both the standard GP and GP with simplification 
are detailed in table 5.1.

Table 5.1: Settings/Parameters used for all Genetic Programming systems

\begin{tabular}{|r|l|r|l|}
\hline Parameter & Value & Parameter & Value \\
\hline Mutation & $30 \%$ & Population Size & 500 \\
Elitism & $10 \%$ & Generations & 100 \\
Crossover & $60 \%$ & Maximum Depth Limit & 6 \\
\hline
\end{tabular}

In addition to the parameters in table 5.1, GP with simplification requires another three parameters. Recall that frequency dictates how often simplification is invoked. A frequency value of 1 means that simplification is invoked every generation, while a frequency value of 7 would mean that simplification would be invoked every seventh generation, with 6 standard GP generations in between. For this chapter, we use three different frequencies for the GP systems using simplification: Every generation, every 5 generations and every 10 generations. Combined with the standard GP system, this makes four systems total to be tested. All programs in the population are simplified whenever simplification is invoked. We use the same values for Hash order ( $p$ ) [1000077157] and constant precision ( $\delta)$ [1000000] as we did in chapter 4 .

\subsection{Results and Discussion}

Each of the four systems described in the experimental setup were run 50 times each (a total of 200 runs) for both tasks, with each run using a different random seed value (and thus different initial conditions). The aggregate results for all of the runs are displayed in table 5.2, listing averages and standard deviations of several measured characteristics: the fitness of the best program in the final generation (Final Best Fit), the time (in seconds) that system takes to evolve over the 100 generations (Time(s)), and the average size of all the genetic programs throughout all runs (Avg. 
Table 5.2: Aggregate results from Regression 4 , Regression ${ }_{2}$ and Coins tasks, using simplification frequencies of: None, Every Generation, Every 5 Generations and Every 10 Generations.

\begin{tabular}{|c|c|c|c|c|c|}
\hline Task & Frequency & Gens & Best Fitness & Time(s) & Avg. Prog Size \\
\hline \multirow{5}{*}{ Regression $_{4}$} & Without & $96.94 \pm 10.84$ & $2.94 \pm 4.93$ & $1.60 \pm 0.268$ & $35.02 \pm 9.22$ \\
& Every 1 & $98.78 \pm 7.21$ & $1.84 \pm 3.84$ & $1.73 \pm 0.333$ & $23.51 \pm 8.82$ \\
& Every 5 & $97.94 \pm 8.55$ & $2.87 \pm 4.74$ & $1.29 \pm 0.268$ & $25.79 \pm 9.06$ \\
& Every 10 & $99.22 \pm 5.51$ & $2.16 \pm 3.85$ & $1.26 \pm 0.207$ & $26.24 \pm 8.81$ \\
\hline \multirow{3}{*}{ Regression $_{2}$} & Without & $100 \pm 0$ & $401.82 \pm 843.27$ & $1.62 \pm 0.208$ & $36.30 \pm 9.25$ \\
& Every 1 & $100 \pm 0$ & $320.40 \pm 435.97$ & $2.24 \pm 0.369$ & $30.05 \pm 9.00$ \\
& Every 5 & $100 \pm 0$ & $284.21 \pm 552.05$ & $1.48 \pm 0.222$ & $29.88 \pm 8.81$ \\
& Every 10 & $100 \pm 0$ & $429.92 \pm 845.14$ & $1.40 \pm 0.204$ & $30.02 \pm 8.63$ \\
\hline
\end{tabular}

Prog Size).

The aggregate results in table 5.2 are consistent with our results in chapter 4 in showing reduced program sizes and slight variations in the average fitness of solution programs. We also see that the average time that GP takes to run is reduced when using simplification, except when simplifying at every generation. In both tasks, it can be seen that simplification at some frequency is able to achieve a final fitness that is better than that of the standard GP.

\subsubsection{Analysis: Disruption of Existing Building Blocks}

Figure 5.3 displays four numerical-node scatterplots (numerical-node values vs. generations), one for an example individual run of each GP system (without, every 1, every 5 and every 10). All four runs displayed in this way were given the same initial conditions (e.g. initial population and random seed values).

The numerical-node plots for the standard GP system (in figure 5.3a) clearly show that in each run, while the initial distribution of numericalnodes is very uniform (spread out evenly across all the initial value range), "bands" of node values are quickly formed as the evolution progresses. 


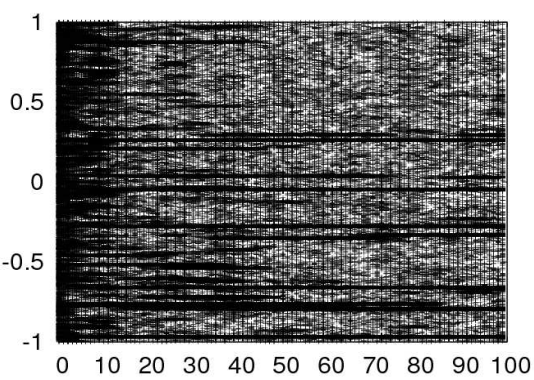

(a) Without

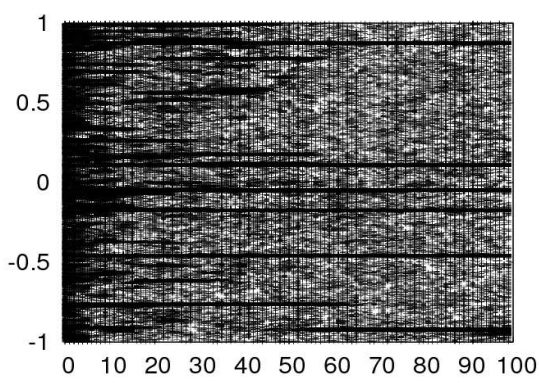

(c) Every 5

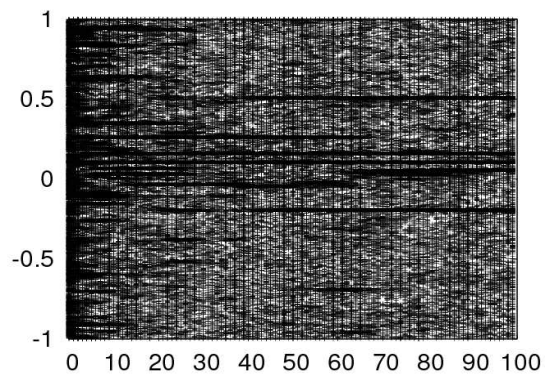

(b) Every 1

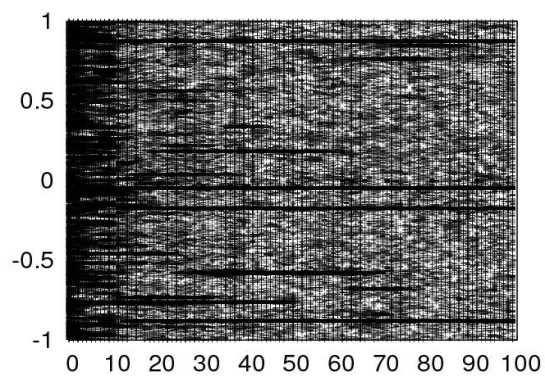

(d) Every 10

Figure 5.3: An example of numerical-node plots (values vs. generations, plotted with a vertical axis range $[-1 . .1]$ ) for each of the four GP systems: without simplification, every generation, every 5 generations and every 10 generations.

This is caused by a relatively small number of numerical-nodes being propagated through the population at each generation, while other nodes are eliminated or dwindle, as a result of the GP operators. These "bands" symbolise the numerical-nodes which are being sustained for long stretches, as they are deemed highly fit by the GP system. These numerical-nodes signify the building blocks we are looking for, as they are nodes which appear to be highly useful to the GP system for constructing solutions, as opposed to merely noise which can be discarded.

However, in the systems that used simplification (shown in figure 5.3bd), many of the bands present in the standard GP run are not present de- 
spite using the same initial conditions. Many numerical-nodes that were deemed fit in the standard GP and promoted throughout the population disappear in the GP systems using simplification. This means that early on in the evolutionary process for these systems, these particular numericalnodes are being eliminated by the program simplification process from the GP population. This behaviour suggests that the simplification procedure disrupts these building blocks during the first few generations, at the first few occurrences of the simplification component being invoked.

\subsubsection{Analysis: Construction of New Building Blocks}
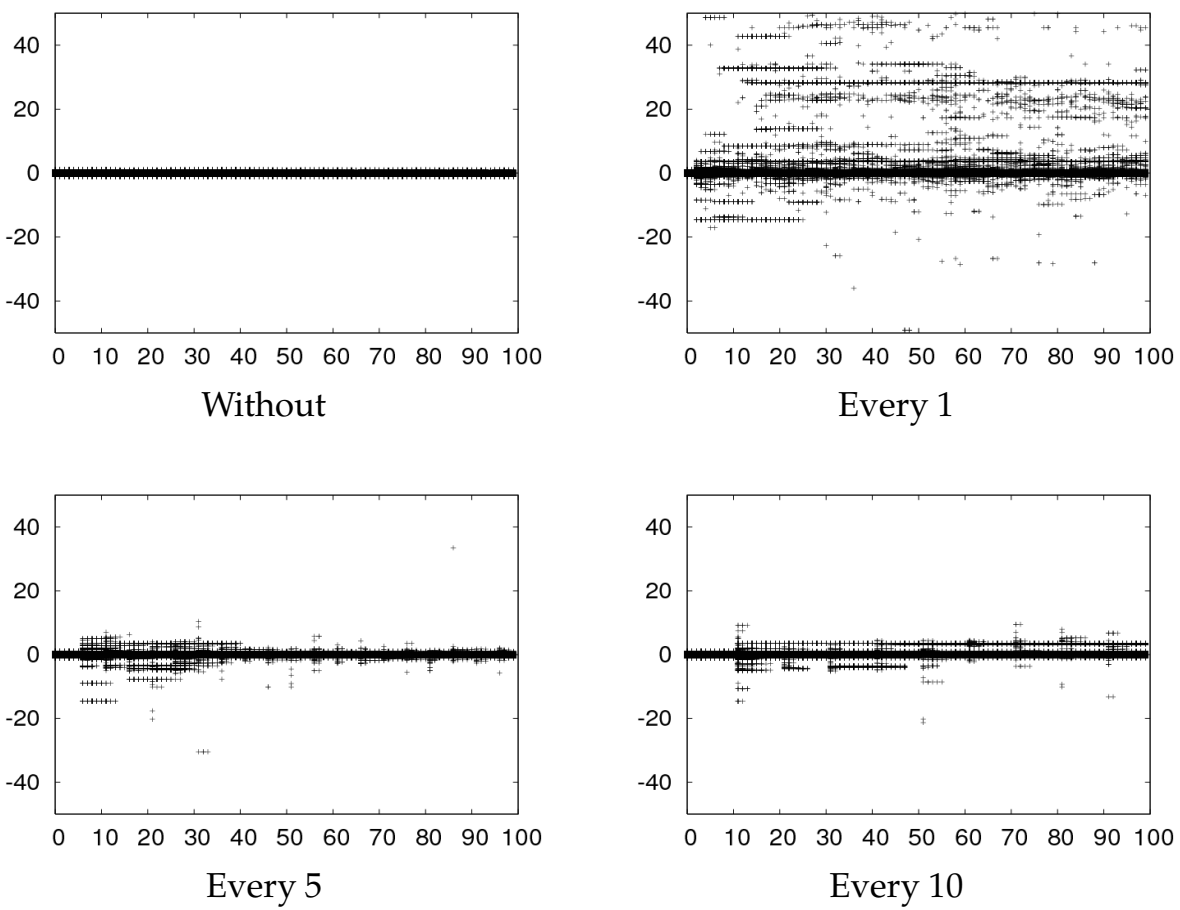

Figure 5.4: An example of numerical-node plots (values vs. generations, plotted for (plotted with a vertical axis range $[-50,50]$ ) for each of the four GP systems: without simplification, every generation, every 5 generations and every 10 generations. 
As the simplification system appears to disrupt building blocks, it would seem that the performance of systems that use simplification should be much worse than the standard GP. From the aggregate results, we know that this is not the case. According to the same plots in figure 5.3, while some bands in the standard GP plots are not present in the GP with simplification system plots, there are also new bands in GP with simplification plots which are not present in the standard GP plots. The creation of these new bands in the systems using simplification indicate that the simplification procedure is not just disrupting old building blocks, but also constructing new and different building blocks, from which the GP system can construct solutions. One may argue that mutation (and not simplification) is the cause of these new building blocks. We present another set of plots (given in figure 5.4) which show the same four plots as in figure 5.3, but plotted within the vertical range on $[-50,50]$ instead of $[-1,1]$. These plots indicate that not only are new building blocks created within the initial numerical-node range, but are also created outside the initial range which can be propagated during the evolution. This creation outside of the initial range can only be the product of simplification as mutation is also restricted to the initial range parameter. This type of creation is particularly clear for GP with simplification at every generation and every 10 generations.

\subsubsection{Effect of Construction/Disruption on Performance}

While these new building blocks are apparent in all cases, the formation of them does not seem to guarantee improved performance over standard GP for a particular run. This is because, like in standard GP, GP with simplification systems are able to be misled by "fit looking" paths in the search space, resulting in sub-optimal solutions being found.

Recall that the initial range used in the original experiments for generating the initial numerical-nodes was $[-1,1]$. This range was used as it was 
Table 5.3: Additional results for Regression ${ }_{4}$, using four different initial numerical-node generation ranges.

\begin{tabular}{|c|c|c|c|c|}
\hline Range & Freq. & Final Best Fit & Time(s) & Avg. Prog Size \\
\hline & Without & $0.002 \pm 0.008$ & $2.844 \pm 0.452$ & $29.743 \pm 3.869$ \\
& Every 1 & $0.005 \pm 0.019$ & $2.864 \pm 0.400$ & $21.766 \pm 2.636$ \\
{$[-10,10]$} & Every 5 & $0.003 \pm 0.009$ & $2.289 \pm 0.271$ & $22.508 \pm 2.198$ \\
& Every 10 & $0.003 \pm 0.010$ & $2.266 \pm 0.276$ & $23.575 \pm 2.554$ \\
\hline \multirow{5}{*}[-20,20]{} & Without & $0.001 \pm 0.002$ & $2.905 \pm 0.411$ & $30.074 \pm 3.831$ \\
& Every 1 & $0.003 \pm 0.008$ & $2.881 \pm 0.404$ & $21.935 \pm 2.668$ \\
& Every 5 & $0.003 \pm 0.006$ & $2.362 \pm 0.285$ & $22.836 \pm 2.635$ \\
& Every 10 & $0.002 \pm 0.006$ & $2.368 \pm 0.275$ & $24.177 \pm 2.618$ \\
\hline \multirow{5}{*}[-100,100]{} & Without & $0.167 \pm 1.575$ & $2.945 \pm 0.445$ & $29.557 \pm 4.180$ \\
& Every 1 & $0.013 \pm 0.049$ & $2.872 \pm 0.373$ & $21.259 \pm 2.673$ \\
& Every 5 & $0.515 \pm 3.556$ & $2.412 \pm 0.329$ & $22.860 \pm 2.791$ \\
& Every 10 & $0.135 \pm 1.245$ & $2.438 \pm 0.327$ & $24.390 \pm 3.292$ \\
\hline & Without & $0.236 \pm 1.494$ & $3.014 \pm 0.456$ & $29.589 \pm 3.643$ \\
& Every 1 & $0.034 \pm 0.116$ & $2.962 \pm 0.403$ & $21.451 \pm 2.632$ \\
& Every 5 & $0.032 \pm 0.212$ & $2.468 \pm 0.318$ & $23.120 \pm 2.805$ \\
& Every 10 & $0.041 \pm 0.168$ & $2.427 \pm 0.311$ & $23.868 \pm 2.709$ \\
\hline
\end{tabular}


deemed "unsuitable" for the given symbolic regression problem $(11 x+50)$, and would make the problem more difficult. [15] showed that a symbolic regression problem's difficulty could be tuned by varying the initial range that these numerical-nodes were initialised in. Table 5.3 displays results from the same symbolic regression problem as before, but using several ranges. Two of these ranges $([-10,10],[-20,20])$ lie between the two constants used in the problem (11 and 50), and so should be "appropriate" for solving this particular problem. We find in table 5.3 that the standard GP performs very well using these ranges, obtaining the "best" fitness out of the four systems (although they are not statistically significantly different). However, the GP systems with simplification still took a shorter time and produced programs that were smaller than the standard GP systems.

In the other two ranges $([-100,100],[-200,200])$, both of which are much larger than the constants needed in the problem, using simplification appears to achieve better fitness than the standard GP at some frequency value. These results suggest that while the performance of the standard GP can be affected significantly by the range in which the numericalnodes are initialised, the systems using simplification are not as strongly affected. By using simplification, the sensitivity of the GP system toward the numerical-node range used may be lessened, as the simplification systems can construct new numerical-nodes outside of the initialised range. In these types of cases, where the numerical-node range is inappropriate for the particular problem, the construction of these new building blocks has a highly positive effect on the GP system. This behaviour would make using simplification particularly useful for tasks in which no prior knowledge for "correct" parameter values (or indeed even the range of values) for the GP system are known.

Figure 5.5 displays a plot of the number of distinct numerical-nodes present in each generation, averaged over all $50 \mathrm{GP}$ runs. The trend of all the GP systems was to have a large initial number of distinct numericalnodes which are quickly refined to a much smaller number by the GP op- 


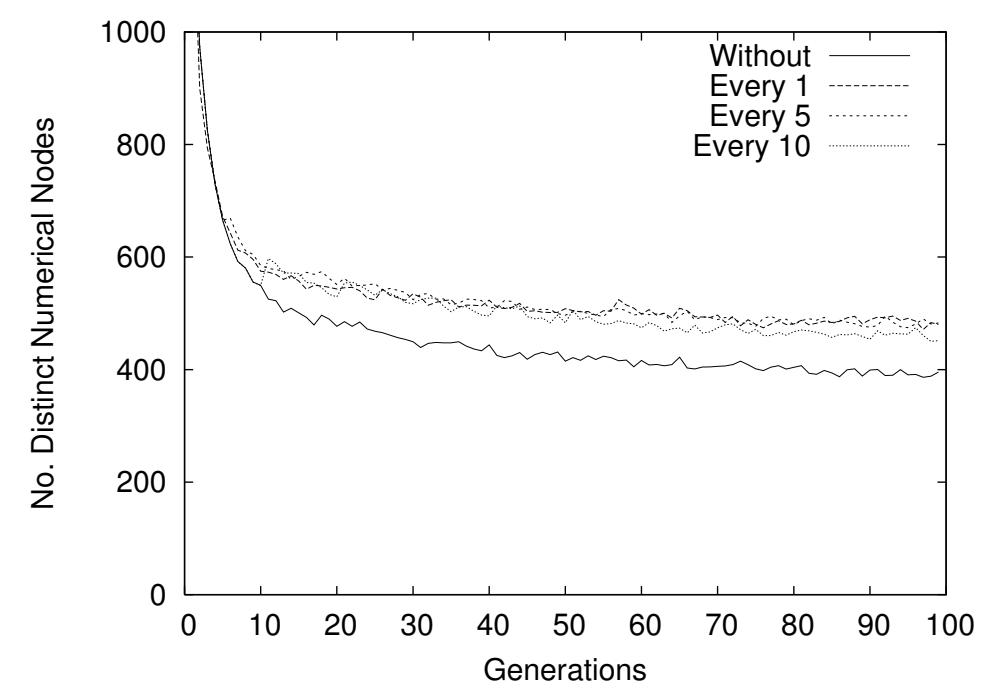

Figure 5.5: The number of distinct numerical-nodes present in each generation, averaged over all 50 GP runs, for the $11 x+50$ problem.

erators. In all of the systems that utilise simplification, it can be seen that on average, the number of distinct numerical-nodes present in each generation is higher than the standard GP for a majority of the GP evolution.

This is caused by the simplification procedure's ability to alter existing numerical-nodes, which in turn creates new numerical-nodes and building blocks. This periodical creation of new numerical-nodes results in more diversity in the numerical-node population. Even though standard GP has an overall larger pool of nodes available (as the average program size is larger in standard GP), systems using simplification provides access to more variations in "genetic material". The added diversity from simplification appears to overcome the loss of genetic material that may occur when existing building blocks are disrupted. The net effect is that the GP population maintains some diversity throughout the GP run, and thus simplification does not lead to premature convergence of the populations. This diversity effect of simplification may help explain why the GP systems employing simplification do not lose any effectiveness despite re- 
moving code from the GP populations, and may actually slightly improve the effectiveness in many cases.

\subsection{Chapter Summary}

The major goals of this chapter were to investigate whether the use of simplification resulted in the disruption of building blocks during a GP system's run, whether using simplification could result in new building blocks being constructed, and whether the positive effects of the new building blocks would outweigh the negative effects of having previous building blocks disrupted. To achieve these goals, we tracked numerical-nodes during individual GP system runs for four GP systems (the standard GP and three GP systems using simplification every generation, every 5 generations and every 10 generations) on a heavily constant-dependent symbolic regression task. The values of every numerical-node in each generation, as well as a count of distinct numerical-node values in each generation were recorded during the runs.

Through analysis of the data using graphical plots, it was found that, disruption of existing building blocks does occur early in the GP evolution, as a result of simplification being invoked on the GP population. However, it was also found that using simplification resulted in new numericalnodes being constructed, which were not present in the GP system before. Some of these new numerical-nodes were created outside of the initial numerical-node range, allowing the GP system to more easily explore more parts of the problem search space. While not all newly formed numericalnodes survived more than a few generations, some new nodes were propagated through the population, constituting new building blocks. Since GP systems using simplification do not produce worse programs than the standard GP (as shown in chapter 4), the new building blocks must counter the effects from disruption of the old building blocks.

Additional results using different initial numerical-node ranges (in ef- 
fect adjusting the difficulty of the task) showed that using simplification resulted in the GP system performing better (and with more stability) than the standard GP in situations where the initial range was highly unsuitable for the problem, suggesting that the creation of new building blocks is highly useful in these situations. This creation is particularly good for problems in which we do not have a lot of prior knowledge, and thus cannot reliably set the GP parameter ranges.

These results provide evidence to help alleviate concerns that using a simplification component may hinder building blocks within a GP population and hence the performance of the GP system. Counts of the number of distinct numerical-nodes in each generation show that GP systems with simplification display increased diversity in the numerical-nodes available to the system.

While analysis on numerical-nodes has provided some insight into the effects of simplification on GP building blocks, it is clear that these interactions need to be further investigated. In the next chapter, we will continue our investigation using a more general and more complex form of building block: the fixed-depth subtree. 


\section{Chapter 6}

\section{Fixed-Depth Subtree Building Blocks}

\subsection{Introduction}

Until now we have been working solely with numerical nodes, which have provided some initial insight into the effects that simplification has on simple building blocks within GP populations. While our results did suggest that simplification does have an effect on the disruption and construction of these simple building blocks, it is clear that GP is not merely an engine to propagate singular numerically-valued nodes to solve problems. We need to look at more complex forms of building block. In this chapter, we will look at a more complex model: using/tracking subtrees of a (small) fixed-depth.

\subsubsection{Chapter Goals}

This chapter aims to build upon work we performed in chapter 5, and investigate/analyse the effects of using online simplification during the evolutionary process using a more complex building-block model. More specifically, we will investigate the following issues: 
- how building-blocks within GP can be represented using a more complex model than numerical-nodes, while still being feasible to perform empirical analysis;

- how we can visualise and interpret these more complex buildingblocks as they are tracked through the GP evolution;

- whether utilising a simplification component in a GP system disrupts potential building blocks in the GP population;

- whether simplification creates new building blocks in a GP population with which to form better solutions; and

- whether the creation of new building blocks can overcome the negative effects of building blocks being disrupted, and result in more accurate GP solutions.

\subsection{Fixed-Depth Program Subtrees}

While there are apparent trends when analysing at the simplest level of building blocks (numerical-nodes in chapter 5), we need to expand our analysis to larger, more complex building blocks, in order to see if these observed trends still hold true. The numerical-node model purely focused on the simplest possible form of building block. It is a natural extension of our numerical-node building block work to track subtrees of larger size, and in a similar manner analyse their behaviour inside a GP evolution run.

Tracking and analysing schema in GP programs is very time consuming and realistically infeasible. This has restricted building block analysis of GP largely in the realm of theory, which is beyond the scope of this thesis. What we want here is a simple estimation of a building block that is relatively easy to track and analyse, yet more complex than the numericalnode model we have used previously. Fixed-depth subtrees provide us with an answer. 
Numerical-nodes were simply a subset of the smallest possible subtree, the single node. In this work, we will be tracking the entire set of single node subtrees (not only numeric nodes, but also feature terminal nodes).

While there has been previous work which argues that building blocks are rooted subtrees (see page 26) because subtrees can have their most pronounced effect on the program when they are rooted, we have decided not to limit the subtrees being tracked to those that are rooted. Recent works have been split over their use of rooted and general subtrees, with no clear consensus. Subtrees which may be important to the overall performance of the program, such as those that construct important numeric constants may only exist on the "lower" parts of the tree. We believe it is dangerous to ignore these subtrees and thus we will track and analyse all subtrees of a GP program.

More specifically, we are concerned with subtrees of a specified depth (e.g. subtrees of depth 1, or subtrees with depth less than 4). Figure 6.1 shows fixed-depth subtrees of various depths in an example program. In similar fashion to when we were using numerical-node building blocks, we will identify these subtrees in each program and convert them to numeric values in order to create a visual representation of building blocks in each generation as the GP evolution progresses. Using this visual plot, we can observe and track subtrees as they are affected by the genetic operators, and algebraic simplification. In this way, we can make further progress in determining how simplification affects the potential building blocks within GP populations.

\subsubsection{Identifying Subtrees}

After a GP run has been completed, every program in each generation is processed to identify all of the subtrees of size 1,2 and 3. This is done by using each node in a program as a starting point (or root) of a subtree, then following all child node pointers until the specified depth has been 


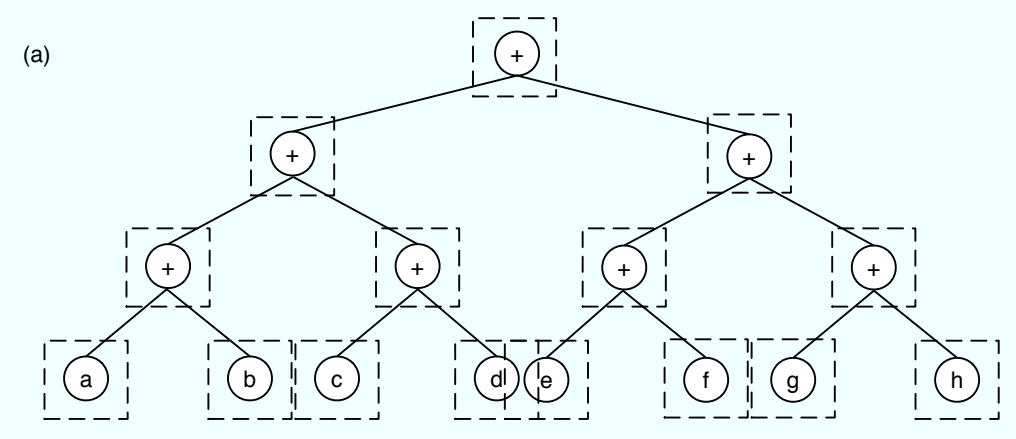

(b)
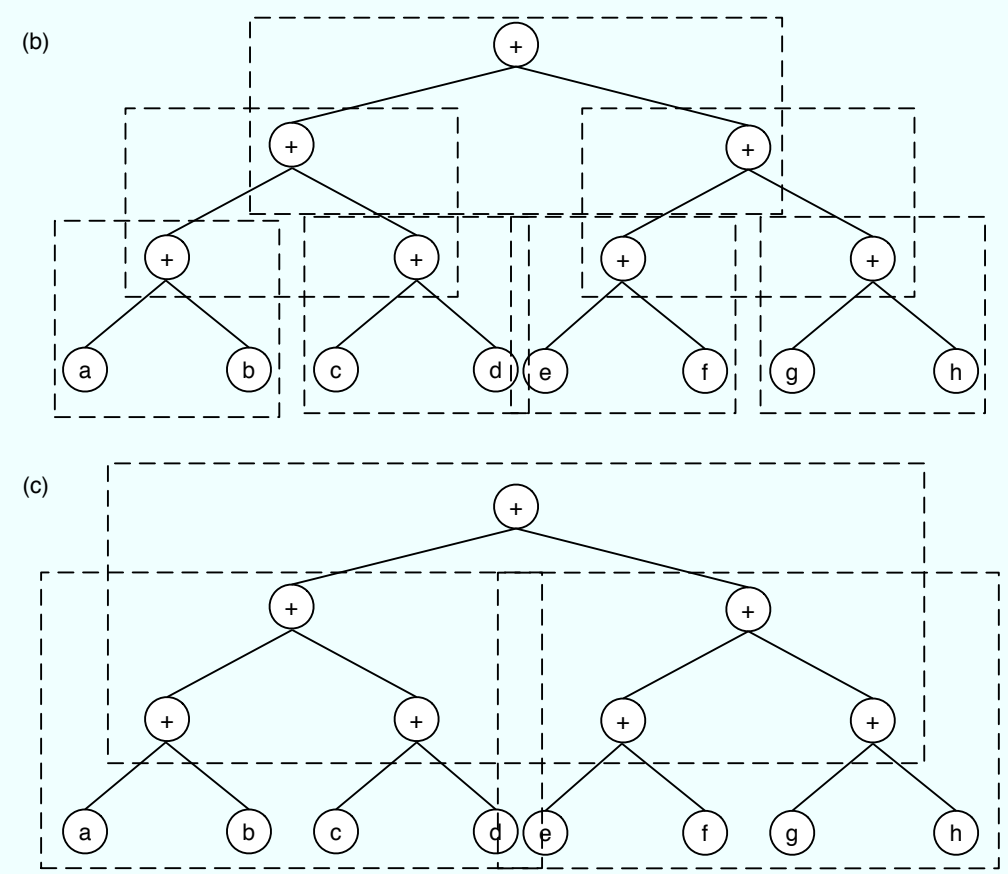

Figure 6.1: Three figures depicting subtrees of various sizes for the example program $(+(+(+a b)(+c d))(+(+e f)(+g h)))$, each subtree is highlighted by a dashed-edge border. (a) size 1 , (b) size 2 , (c) size 3

reached on each path. The process stops either when the specified depth is reached or when there are no more child nodes to traverse to. A basic example of this process is given in figure 6.2. 


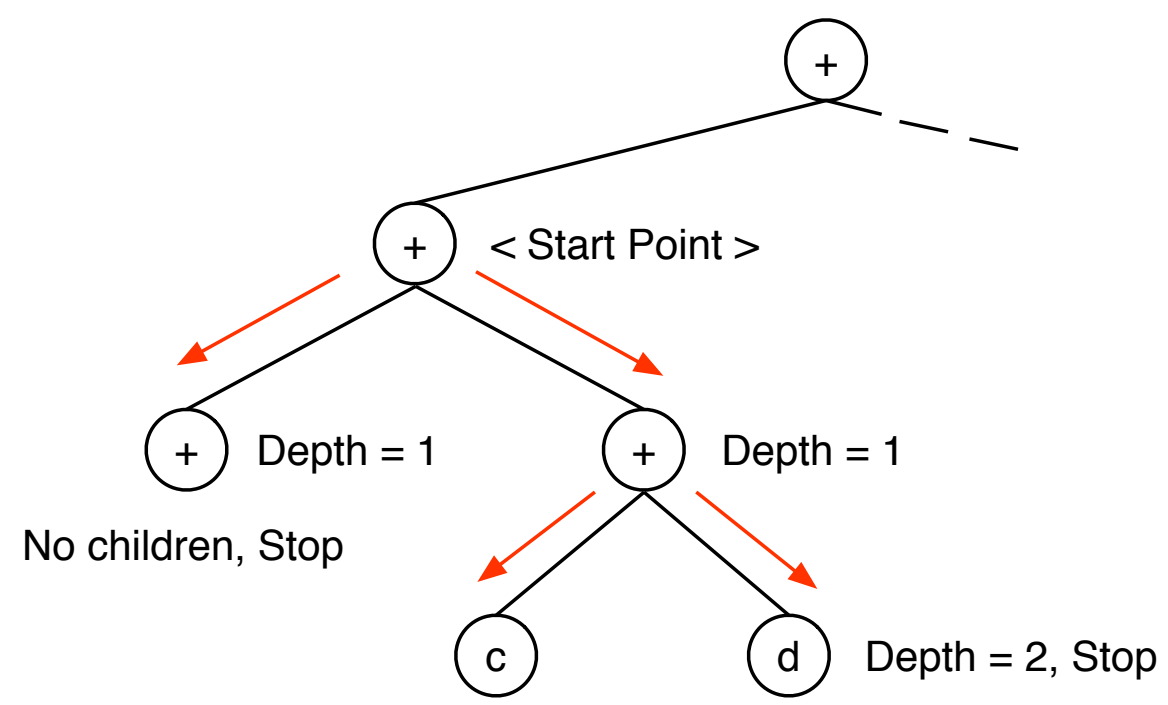

Figure 6.2: Example of identifying a subtree of depth 2

Note that when identifying the subtree, if the process cannot reach the required depth following any of the child node paths, then it is discarded, since the resulting subtree is not of the required depth.

\subsection{Visualising Fixed Size Subtrees in GP}

What made numerical-nodes easy to put into a visual form was the fact they were already numerically valued. This meant that the numericalnodes in each generation could be plotted for each generation in the GP evolution, giving a visual flow of the building blocks throughout the GP evolution. Similar building blocks were clustered together, allowing for identification of large numbers of almost identical building blocks. In this chapter, using larger building blocks which contain multiple (often non-numerical) nodes, creating a visual diagram is not as trivial. Here we explore some possible approaches for visualising the fixed-depth subtree building blocks. 


\subsubsection{Using a Global Indexing Table}

In this first approach, we maintain a global table which indexes all subtrees encountered so far in the GP evolution. The subtrees that are identified within a program are searched for in this global indexing table. In addition to the global indexing table, each generation has a smaller, local list associated with it. This local list is a list of indexes, each of which refer to a subtree that is contained in the global indexing table. These local lists keep track of exactly which building blocks were present in each generation of the GP evolution.

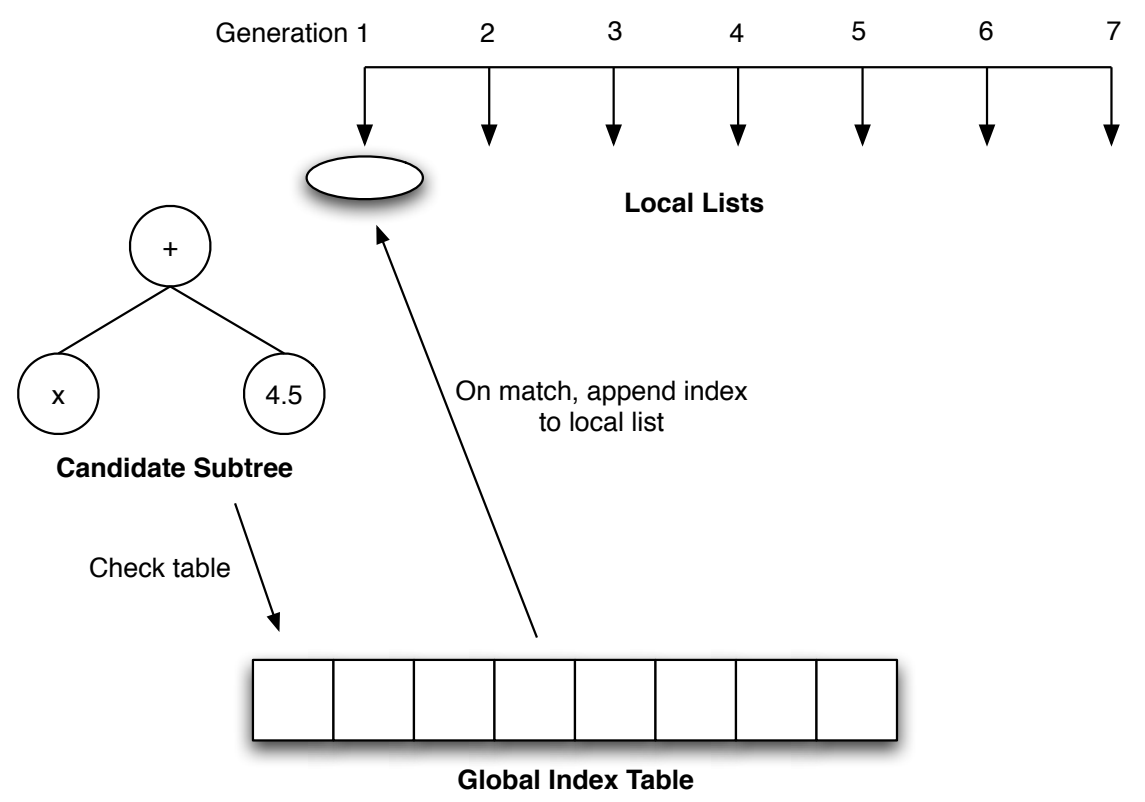

Figure 6.3: Simple example of using a global index table to process fixeddepth subtrees. In this example, the candidate subtree is found in the global index.

As each subtree is identified, the global indexing table is consulted to see if already exists in the table (meaning we have encountered the same subtree before). If there is a match, we use the index value of the subtree in 
the global table and append that to the local list for the current generation. If no match is found, the subtree is added to the global table and the index value of the newly added subtree is appended to the current generation's local list. A diagram for each situation is shown in figures 6.3 and 6.4.

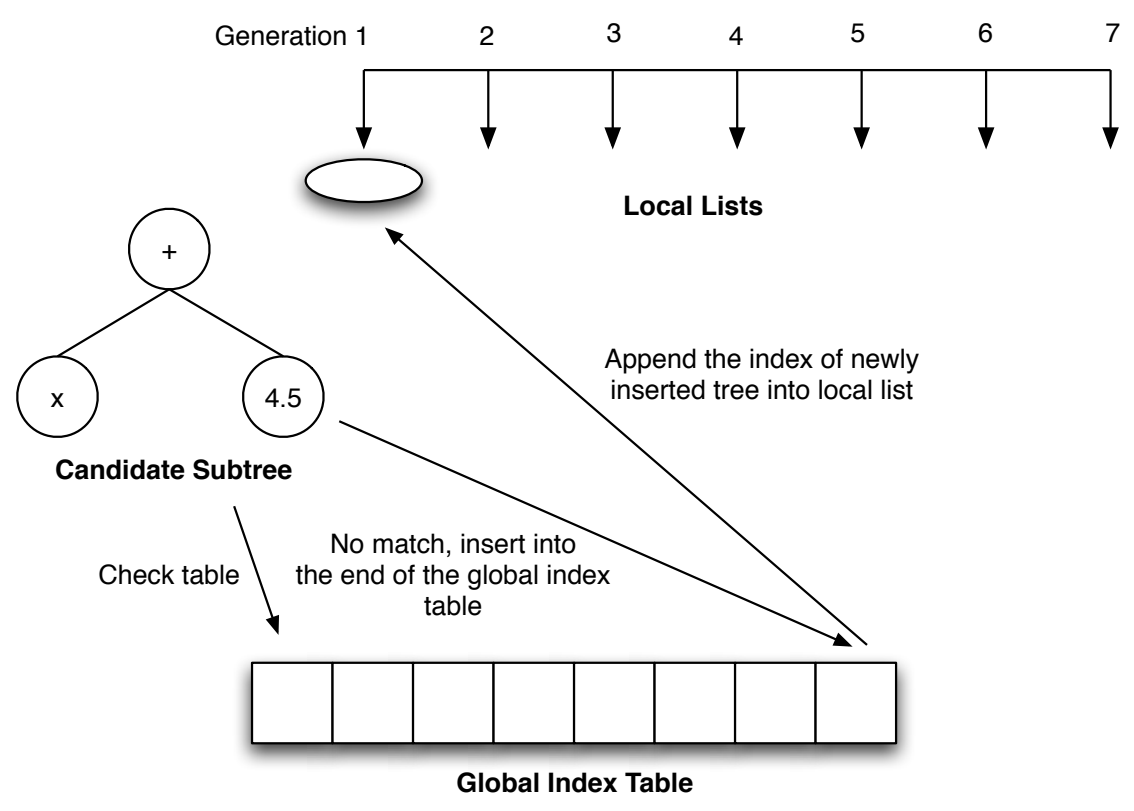

Figure 6.4: Another simple example of using a global index table to process fixed-depth subtrees. In this example, the candidate subtree is not found in the global index.

Through this process, each unique subtree is allocated a unique numerical value as needed. The local lists allow us to monitor subtrees from generation to generation. By plotting the values contained in the generation local lists, we can form a visual scatterplot of the subtrees contained in each generation as the GP evolution run progresses.

However, this approach is limited by the fact that similar subtrees are not guaranteed to be adjacent in index value. Furthermore, a subtree may be given two different index values in plots of two separate runs, as the index value is allocated on a "first-come, first-serve" basis. One approach 
would be to write the global indexing table out to file and reuse the same global table for multiple runs, although with the extremely high number of possible subtrees, this is highly inefficient (due to the heavy use of secondary storage).

A better approach would be to assign each subtree a value through use of a mapping/hash routine. While this may lead to some collisions, a case which is extremely detrimental to the integrity of the analysis, there are several benefits. Firstly, direct comparisons can be made between runs, such as identification of commonly grouped subtrees (which may help identify building blocks that are commonly used to form the solution). Secondly, the speed of the analysis is greatly increased, as a slow global index becomes unnecessary to assign unique subtree values. We investigate the possible use of hash functions next.

\subsubsection{Using a Hashing Function}

Instead of using a global indexing table, a much faster approach would be to use a hash function to convert each fixed-depth subtree into a (unique) numerical value. These numerical values could then be plotted (possibly requiring normalisation first) in a similar fashion to what was used for numerical-nodes.

A suitable hash function for this purpose is not trivial, as there are some requirements that a hash function would ideally fulfill. We identify the following requirements as important:

1. Each unique subtree corresponds to a unique numerical hash value.

2. Smaller differences in structure between two subtrees equates to a smaller difference in hash values. Similarly, larger differences in subtree structure equate to larger differences in hash values.

3. Related to (2). Structure differences higher in the subtree (closer to the root node) correspond to larger differences in hash values than 
structure differences lower in the subtree (closer to the leaf nodes). This is supported by work in [35] showing that changes further away from the root node influence the fitness of the program less than changes closer to the root.

Initially we considered a few already existing hash functions which could possibly be used for hashing the subtrees. Firstly, we considered the Algebraic Equivalence Hashing method described in chapter 4. However, as this would equate subtrees that are structural different but functionally equivalent, it violates the first hashing requirement.

Realising that the subtrees can also be respresented by a string (e.g. (+ f0 (- $0.2 \mathrm{f0}) \mathrm{fl}$ ) ), we considered existing hash functions that can hash strings or collections of bytes. One such hash function considered was CRC32 (32-bit Cyclic Redundancy Check) hashing [63]. CRC32 is a popular and well studied hash function used as a checksum to ensure data integrity. Computation for CRC32 is similar to arithmetic division, but uses remainder as the result while the quotient is discarded. It is widely used as it is easy to implement in binary hardware and is well understood mathematically.

However, although CRC32 does an excellent job of hashing different subtrees to different hash values and limiting collisions, it is not designed to keep similar strings to similar hash values and often will map similar strings to very different hash values. This is against what we are looking for in a hash function (as stated in second hashing requirement). A review of other existing hash functions makes it clear that we will need to construct a new hash function specifically for the task of hashing GP subtrees to numerical values.

\subsection{FST-Hash: Hashing for Fixed-Depth Subtrees}

To address these hashing requirements, we developed a method called FST-Hash (Fixed-SubTree-Hash). FST-Hash is a hashing function designed 
around the three hashing requirements. The hashing process is made up of two steps. The first produces a level ordering of the nodes in the subtree. The second takes this ordering and translates each node into a numerical sequence. The concatenation of these numerical sequences gives us the final hash values.

\subsubsection{Producing a Level Ordering}

When a tree-based GP program is printed as a program string, it is done so in a prefix ordering (i.e. the operator, followed by its arguments). However, in our approach, we wish to produce a level ordering, where each depth or level is printed out in ascending order. This means that we obtain an order sequence where the root node (depth 0 ) is printed out, followed by all the nodes at depth 1, followed by all the nodes at depth 2 etc. Figure 6.5 shows the difference between a prefix ordering and a level ordering on an example GP program tree.

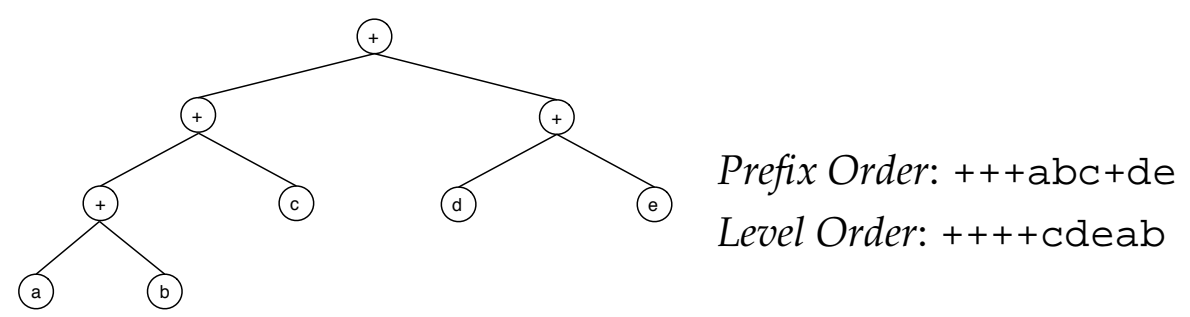

Figure 6.5: Prefix and Level ordering of nodes for an example program.

The motivation behind using this ordering is to address the third hashing requirement. That is, node differences closer to the root should result in larger hash value differences than node differences further down the subtree. By using a level ordering, we have a sequence in which nodes closer to the root are earlier (i.e. larger) in the hash value. Figure 6.6 shows an example program followed by two programs which differ from the example by one node. The left program has had a low level operator + altered into $\mathrm{a}-$, while the right program has had the root node changed. 
Note that while a + node is being changed into a - node in both cases, the left case only results in a relatively small change in hash value, while the right case displays a larger change in hash value.

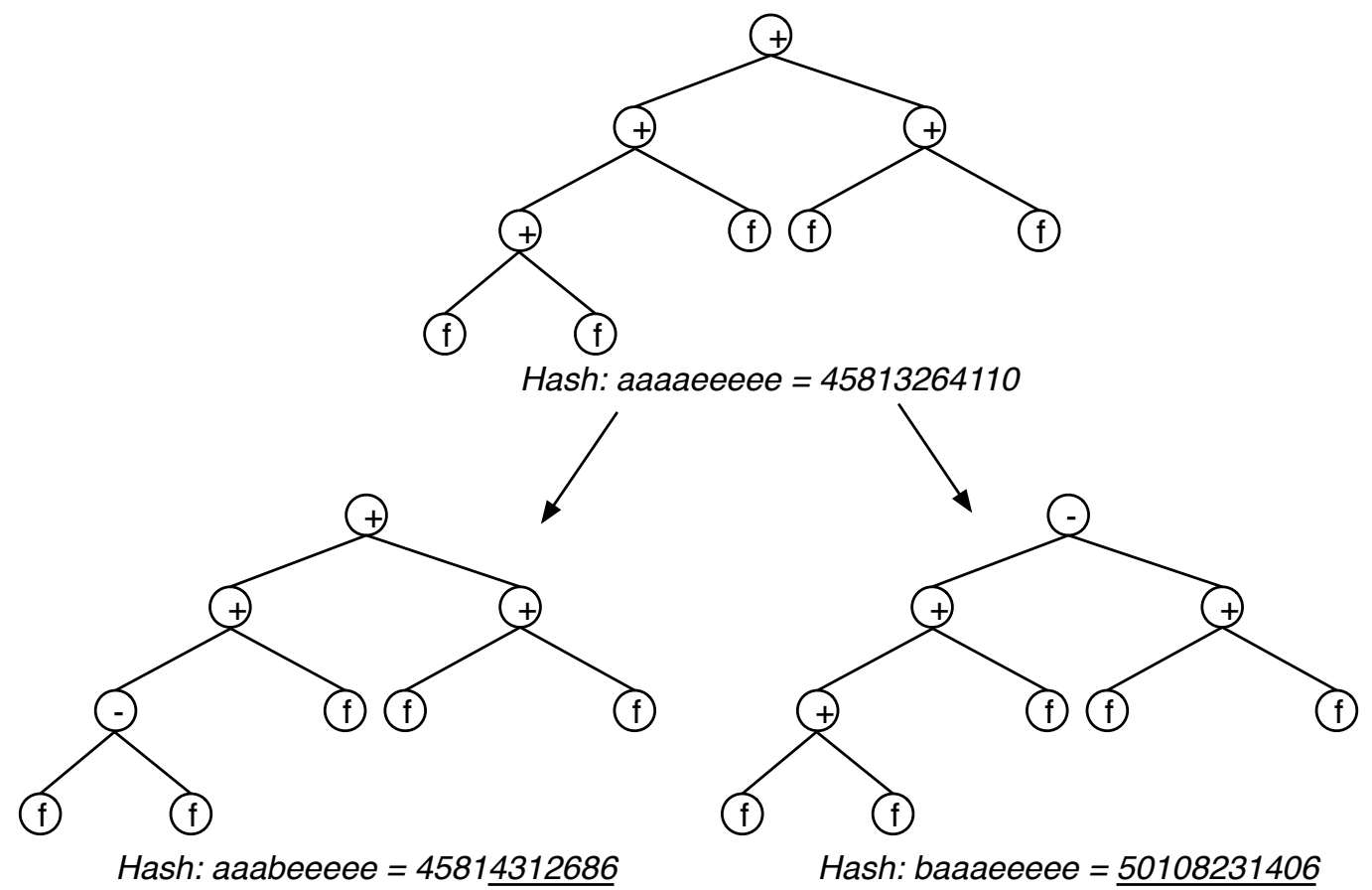

Figure 6.6: How differences in structure at different levels affects the resulting hash value. Underlining shows where the hashes differ from the original program tree's hash. These hash values will be explained in section 6.4 .2

Obtaining a level ordering given a particular GP program is fairly straightforward. We begin by adding the root node to a queue. We then take the next node off the queue and append it to our ordering. Then we add the node's children onto the end of the queue. We continue this process until there are no more nodes on the queue. In this way we visit each node in the program tree once and produce a level ordering. The pseudo-code for this procedure is given in figure 6.7. 


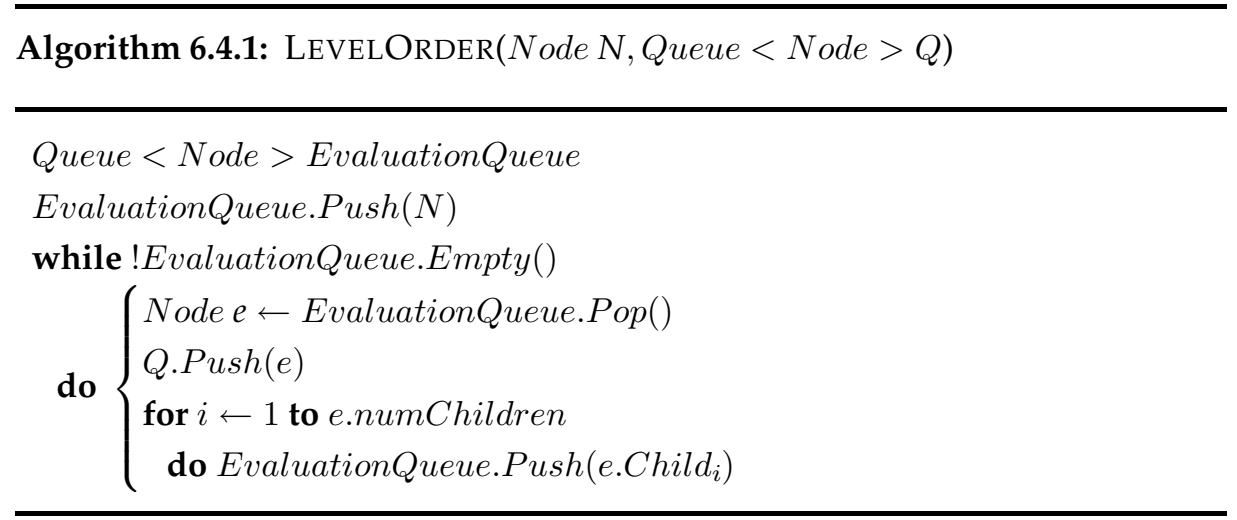

Figure 6.7: Pseudo-code producing a level ordering from a GP subtree.

\subsubsection{Translating the Ordering to a Hash}

Now that we have a level ordering for the nodes in the GP subtree, we need to translate those nodes into numerical sequences in order to get the final numerical hash value. This is achieved by simply enumerating the number GP nodes which provides a mapping from the nodes into a numeric sequence. In more detail, we handle each node in the level ordering as follows.

Numeric Valued Terminals: In this approach we address floating point valued nodes as opposed to integer or rational values. As floating points can often have differing numbers of significant digits (e.g. 0.222 [3 significant digits] vs. 0.100 [1 significant digit]), we specify a precision parameter which specifies a set number of decimal places (d.p.) we use when translating these nodes. We use a precision of 2 d.p. in our experiments, which means that a numeric node of value 1.2332 is translated to 123 , whilst a numeric node of value 0.498723 is translated to 050 (as the 3rd decimal place is above 4 , so we round up). Note that if the numeric value has fewer than 2 d.p., the rest of the sequence is filled in with zeros (e.g. 0.1 translates to 010 ). 
Operators: For these types of nodes, the process is straightfoward. We can assign each of these operators a value. As the decimal digits $(0-9)$ are already used for the numeric valued terminal nodes, we assign each of the operators an alphabetic character $(a-z)$. For our experiments, we have the following mapping: $+\rightarrow a,-\rightarrow b, \times \rightarrow c, \div \rightarrow d$. In this work, we are not using an if $<0$ operator, and so it is not handled in our translation (though we would simply associate the next available unused alphabetic character to it, just like the other operators).

Feature Terminals: These are processed in the same way as the operator nodes. However, we do not reuse any of the alphabetic characters that have already been used by the operator mappings. In our experiments, as we are only concerned with one variable symbolic regression, we have the following mapping: $f_{0} \rightarrow e$.

These mapping settings are summarised in table 6.1. Note that in this work, we are only concerning ourselves with a maximum subtree size of 3. The choice of depth and decimal place precision determine how large hash values can become. We have found using our settings that in the vast majority of cases the calculated hash values are well under the maximum size of a 64-bit unsigned integer, and so implementation of this approach is not an issue.

\subsubsection{Plotting Hash Values}

Note that for our experiments, we use the digits $0-9$ and the alphabetic characters $a-e$, making a total of 15 different characters used. Any sequence that result from this process will essentially be a number in base 15. In order to plot the hash values produced by FST-Hash, we convert these sequences into an equivalent base-10 number (decimal) [27]. The pseudo-code for this function is presented in figure 6.8 , and an example is given in figure 6.9.

Numerical-nodes consisted of only numerically valued singular termi- 


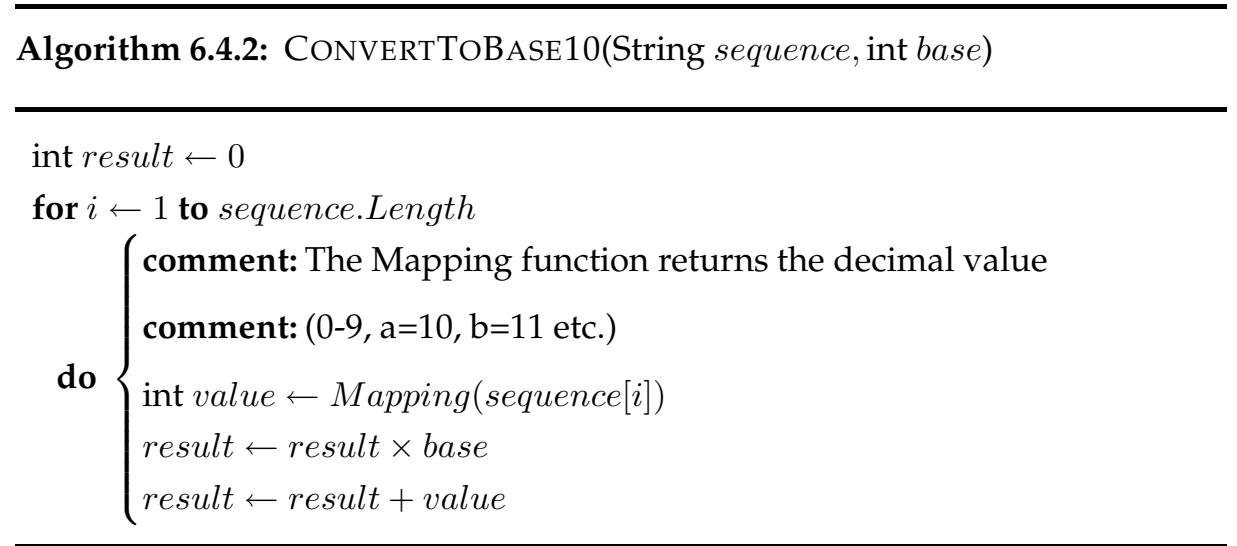

Figure 6.8: Pseudo-code for converting an arbitrary base number to base10.

\begin{tabular}{rll}
\hline Step & Digit & Result \\
\hline Initial & - & 0 \\
Multiply by 15 & - & 0 \\
Add 10 & a & 10 \\
Multiply by 15 & & 150 \\
Add 11 & b & 161 \\
Multiply by 15 & & 2415 \\
Add 12 & c & 2427 \\
Multiply by 15 & & 36405 \\
Add 1 & \multirow{2}{*}{1} & 36406 \\
Multiply by 15 & & 546090 \\
Add 2 & 2 & 546092 \\
Multiply by 15 & & 8191380 \\
Add 3 & 3 & 8191383 \\
\hline
\end{tabular}

Figure 6.9: Example converting the base-15 number abc123 into decimal (base-10). The result here is 8191383 . 
Table 6.1: Summary of mappings used to translated level-ordering to a numeric sequence

\begin{tabular}{|c|c|}
\hline Node & Mapping \\
\hline \multirow{5}{*}{ Numerical Nodes } & (Only using a specified number of decimal places) \\
& $0 \rightarrow 0$ \\
& $1 \rightarrow 1$ \\
& $2 \rightarrow 2$ \\
& $3 \rightarrow 3$ \\
& $4 \rightarrow 4$ \\
& $5 \rightarrow 5$ \\
& $6 \rightarrow 6$ \\
& $7 \rightarrow 7$ \\
& $8 \rightarrow 8$ \\
& $9 \rightarrow 9$ \\
\hline Operators & $+\rightarrow a$ \\
& $-\rightarrow b$ \\
& $\times \rightarrow c$ \\
& $\div \rightarrow d$ \\
\hline
\end{tabular}

nal nodes. This means that while there were often hundreds or thousands of values that were plotted on each scatterplot, it was still clear which numerical-node building blocks were popular and which were rare. However, with even slightly larger subtrees (the size 2 and 3 we use in this thesis), there are a far greater amount of subtrees that need to be plotted (in the millions). If we use the 2-dimensional scatterplot approach that we used for numerical-nodes, we obtain a plot that is cluttered and very hard to interpret (see figure 6.10).

One way to address this is to reduce the population size used in the GP system. This greatly reduces the number of subtrees that need to be processed and plotted in each generation and hence the resulting scatterplots 


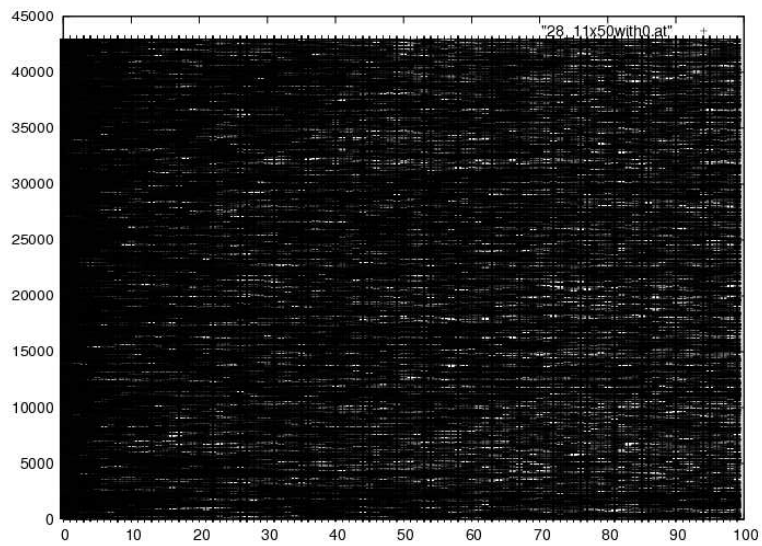

Figure 6.10: 2D plot of fixed-depth subtrees, using CRC32 hash values.
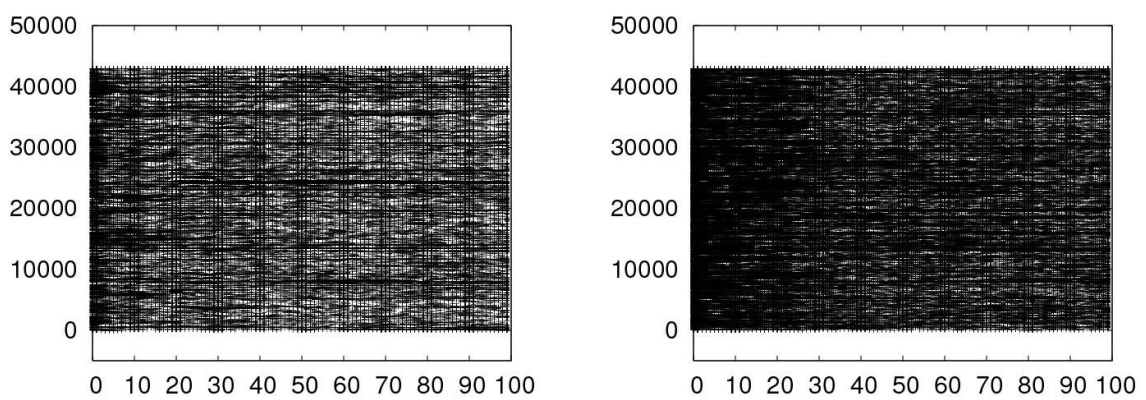

Figure 6.11: 2D plot of fixed-depth subtrees, using CRC32 hash values, using left: Population 100, right: Population 250.

are much clearer. Example plots using reduced populations of 100 and 250 are shown in figure 6.11. However, by reducing the population we are also reducing the appropriateness of the analysis as we are no longer using "typical" GP settings (which we have used in our other experiments).

Instead, we extend the normal scatterplot by plotting each subtree in each generation only once ( $x$-axis: generations, $y$-axis: subtree hash value), with the $\mathrm{z}$-axis representing the frequency of that particular subtree. We further use a colour gradient to illustrate the frequencies. Using this approach we can view the popularity of certain subtrees as well as how they are affected during the GP evolution. In using this approach, we no longer 
have to add random noise to each value, as heavily populated subtrees will just be represented by a higher "peak" and different colour.

\subsection{Experimental Setup}

For experimentation, we use two symbolic regression problems and a classification problem. The first symbolic regression task we use is the Regression ${ }_{4}$ task (described in chapter 3), which is used as a fairly simple task so that complications or effects that may be caused by problem difficulty may be ignored. This is the same problem that we used for analysis in chapter 5 for numerical-node analysis, so we use it again here in this work to allow for comparison. The second symbolic regression problem we use is the Regression ${ }_{2}$ task (also described in chapter 3). This was selected as a more difficult problem to contrast the first problem we used, and is used for the higher level analysis. The classification task we use is the coins classification dataset (chapter 3). We chose to include a classification task in order to verify that our findings were not limited solely to the symbolic regression problem domain.

We used the usual experimental setup (i.e. terminal set, function set, crossover/mutation/elitism rates etc.) that we have used throughout this thesis, with only a couple of differences. The first change is that we used a setting of 100 generations of evolution. This gives us a larger view of the behaviour of the fixed-depth subtrees. The second change was the removal of if $<0$ from the function set.

\subsection{Results and Discussion}

Firstly we present results aggregated from all 50 test runs for each task in the experiments, to provide some background information on the nature of these problems and the performance obtainable by each of the four systems. Table 6.2 shows the average best fitness, average evolution time and 
Table 6.2: Aggregate results from Regression 4 , Regression ${ }_{2}$ and Coins tasks, using simplification frequencies of: None, Every Generation, Every 5 Generations and Every 10 Generations.

\begin{tabular}{|c|c|c|c|c|c|c|}
\hline Task & Frequency & Gens & Best Fitness & Time(s) & Avg. Prog Size & Best Accuracy \\
\hline \multirow{6}{*}{$\operatorname{Reg}_{4}$} & Without & $96.94 \pm 10.84$ & $2.94 \pm 4.93$ & $1.60 \pm 0.268$ & $35.02 \pm 9.22$ & N/A \\
& Every 1 & $98.78 \pm 7.21$ & $1.84 \pm 3.84$ & $1.73 \pm 0.333$ & $23.51 \pm 8.82$ & N/A \\
& Every 5 & $97.94 \pm 8.55$ & $2.87 \pm 4.74$ & $1.29 \pm 0.268$ & $25.79 \pm 9.06$ & N/A \\
& Every 10 & $99.22 \pm 5.51$ & $2.16 \pm 3.85$ & $1.26 \pm 0.207$ & $26.24 \pm 8.81$ & N/A \\
\hline \multirow{6}{*}{$\operatorname{Reg}_{2}$ ( Without } & $100 \pm 0$ & $401.82 \pm 843.27$ & $1.62 \pm 0.208$ & $36.30 \pm 9.25$ & N/A \\
& Every 1 & $100 \pm 0$ & $320.40 \pm 435.97$ & $2.24 \pm 0.369$ & $30.05 \pm 9.00$ & N/A \\
& Every 5 & $100 \pm 0$ & $284.21 \pm 552.05$ & $1.48 \pm 0.222$ & $29.88 \pm 8.81$ & N/A \\
& Every 10 & $100 \pm 0$ & $429.92 \pm 845.14$ & $1.40 \pm 0.204$ & $30.02 \pm 8.63$ & N/A \\
\hline \multirow{6}{*}{ Coins } & Without & $99.18 \pm 3.58$ & $0.93 \pm 0.047$ & $1.17 \pm 0.212$ & $27.98 \pm 9.44$ & $0.93 \pm 0.055$ \\
& Every 1 & $99.46 \pm 3.82$ & $0.92 \pm 0.047$ & $1.59 \pm 0.291$ & $21.52 \pm 8.99$ & $0.93 \pm 0.050$ \\
& Every 5 & $99.76 \pm 1.70$ & $0.93 \pm 0.051$ & $1.12 \pm 0.195$ & $22.92 \pm 9.04$ & $0.93 \pm 0.060$ \\
& Every 10 & $97.98 \pm 8.55$ & $0.92 \pm 0.070$ & $1.06 \pm 0.186$ & $23.99 \pm 8.95$ & $0.92 \pm 0.069$ \\
\hline
\end{tabular}

average program size for each of the four systems. These results are consistent with those in chapter 4 , and confirm that GP with simplification can reduce program size and CPU time, while maintaining the effectiveness of GP in finding solutions.

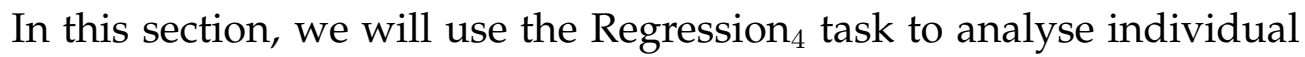
runs in section 6.6.1. We then use all three tasks (Regression ${ }_{4}$, Regression $_{2}$ and the Coins dataset) to perform higher level analysis on the overall effect of program simplification.

\subsubsection{Analysing Individual Runs}

In our numerical-node model in Chapter 5, we used the Regression 4 symbolic regression problem to observe and perform analysis of individual GP evolution runs. In this subsection we perform similar analysis, using the fixed-depth subtree model. First, we will look at fixed-subtrees of depth 1 separately and make comparisons to the results we obtained from using 
the numerical-node model. Then we will analyse fixed-subtrees of depths 2 and 3 to see if there are obvious trends with these larger subtrees.

In this analysis we will display 3D plots of various GP runs, both systems with and without simplification. The bottom axis ( $\mathrm{x}$-axis) represents each GP generation, from generation 1 through to generation 100. The right hand axis (y-axis) represent the FST-Hash values, and so each horizontal "slice" tracks a single subtree through all 100 generations of that GP run. The left hand vertical axis (z-axis) shows the frequency for a particular FST-Hash value (program subtree) in a particular generation. A colour gradient is shown in each plot in the upper right hand corner, showing the corresponding colour pertaining to particular frequency values.

Higher peaks mean that there is a larger number of that particular subtree at that point in the GP run. If a subtree is consistently highly populated (forming a ridge that spans multiple generations) then that subtree is both plentiful in the GP population and has survived through multiple generations of genetic operators. If any new ridges appear during the course of the GP run, then those particular subtrees have somehow propagated into a significant proportion of the GP populations.

\section{Analysis of Fixed-Subtrees of Depth 1}

Using fixed-depth subtrees of depth 1 is a slight extension over using the simple numerical-node model, where we are now incorporating the functional nodes. While we generated plots and observed trends for all 50 runs for each system, we only display plots from four different runs here for space reasons. Although we will illustrate our results using these four example runs, our findings cover all of the 50 runs that we monitored. Figure 6.12 shows plots for these runs using no simplification, while figures 6.13, $6.14,6.15$ show plots for using simplification every 1,5 and 10 generations respectively.

Looking at the plots for runs using no simplification, it is notable that hash value 14 (which corresponds to the feature terminal) is by far the 


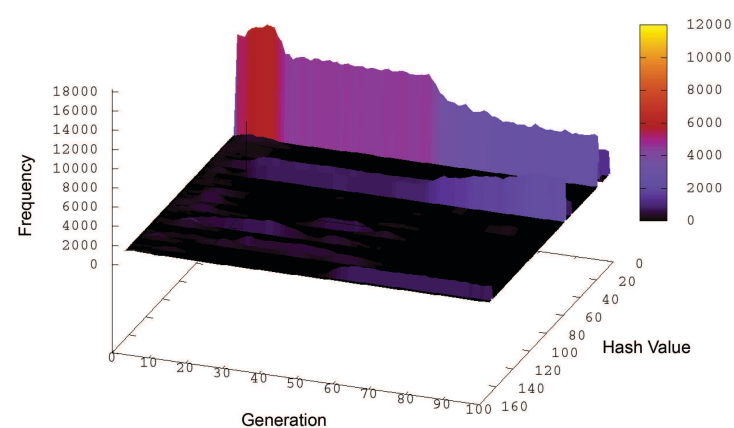

(a)

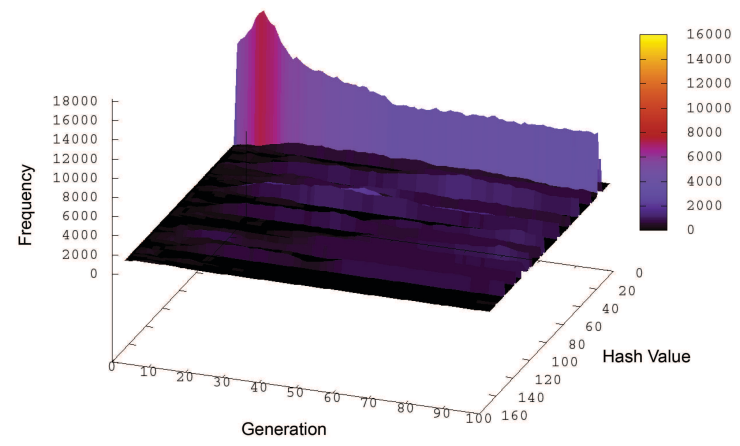

(c)

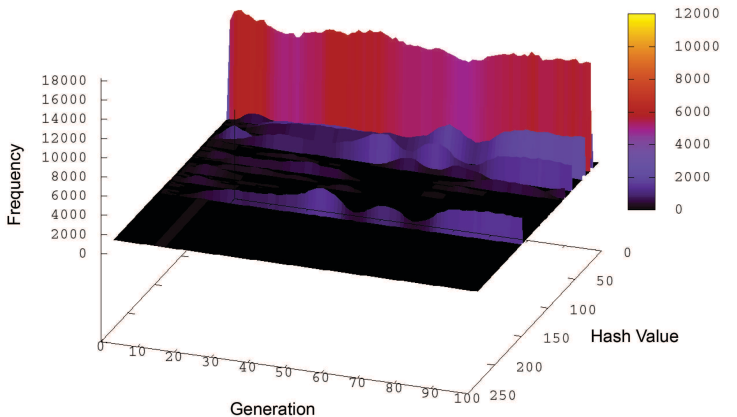

(b)

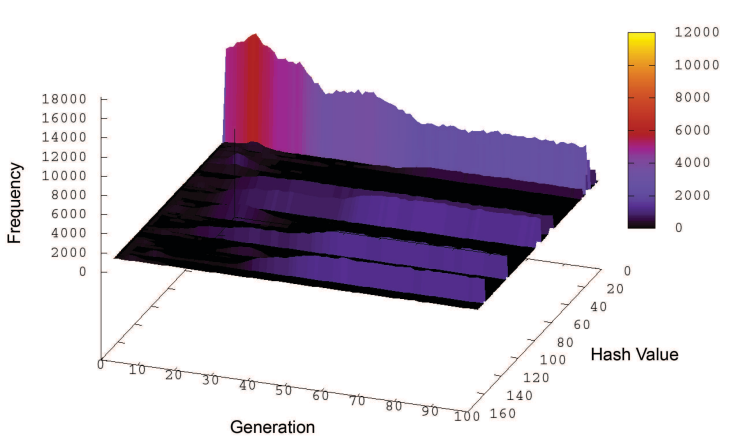

(d)

Figure 6.12: A sampling of 3D plots (run (a) 5, (b) 9, (c) 14 and (d) 29) of fixed-depth subtrees of depth 1, using FST-Hashing, no simplification. Using a smaller plotting range to zoom-in. 
Table 6.3: Top 5 Most Popular Subtrees for Depth 1

\begin{tabular}{|cc|cc|cc|cc|}
\hline \multicolumn{2}{|c|}{ No Simplification } & \multicolumn{2}{|c|}{ Every 1 } & \multicolumn{2}{c|}{ Every 5 } & \multicolumn{2}{c|}{ Every 10 } \\
Hash Value & Freq. & Hash Value & Freq. & Hash Value & Freq. & Hash Value & Freq. \\
\hline 14 & 11874 & 14 & 13974 & 14 & 13920 & 14 & 14046 \\
14 & 14046 & 14 & 14643 & 14 & 12756 & 14 & 12921 \\
14 & 12921 & 14 & 15144 & 14 & 11322 & 14 & 12405 \\
14 & 12405 & 14 & 11220 & 14 & 17079 & 14 & 17607 \\
14 & 18177 & 14 & 11964 & 14 & 11991 & 14 & 13719 \\
\hline
\end{tabular}

most populated node. This is further illustrated in table 6.3 (which also shows that the node is the most popular in all four systems). This table shows the five most populous subtrees, as found using the following method: We take the subtree which is the most populated in any one generation for each of the 50 GP runs. We then take the five most populated of these 50 subtrees. As can be clearly seen, the top five subtrees in each of the four systems is the feature terminal node. This observation can be expected as the feature terminal may occur within a solution multiple times as it attempts to piece together the relatively large coefficient (11 in $11 x+50)$ required for the regression problem.

It also appears that addition (hash value 10) is the most common function used in a majority of the GP runs ( $70 \%$, though not in all the runs). This may be due to the approach that GP can take in formulated $11 x$ (adding feature terminal nodes together), as well as constructing the needed constants.

As in our numerical-node analysis, we find that not using simplification (figure 6.12) restricts the values of numerical-nodes to a specific range (thus the smaller y-axis range). This is clear when comparing the plots in figure 6.12 with those in figures 6.13, 6.14 and 6.15. The subtrees plotted in figure 6.12 are all within a much smaller range than those systems using simplification.

Now we look at the runs where simplification is used. Using simplification ((a), (b), (c) and (d) in figures 6.13, 6.14 and 6.15) results in many 


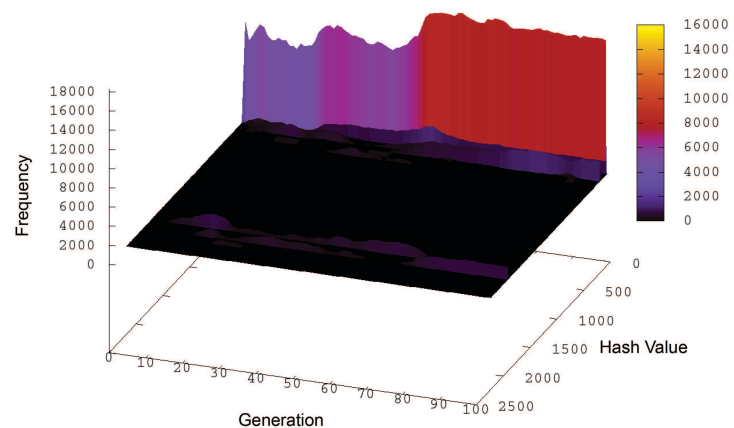

(a)

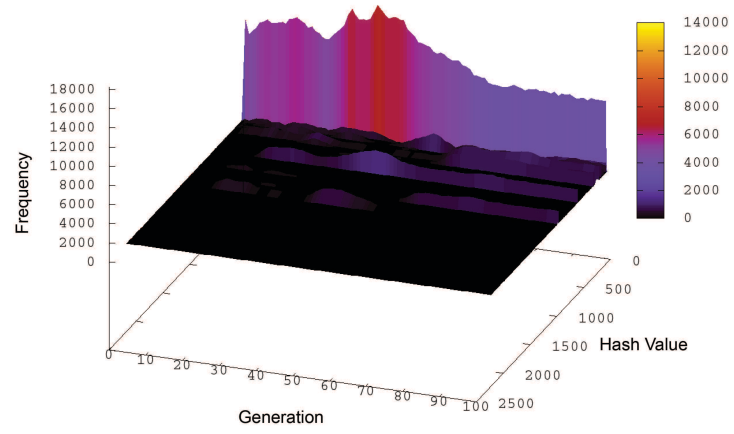

(c)

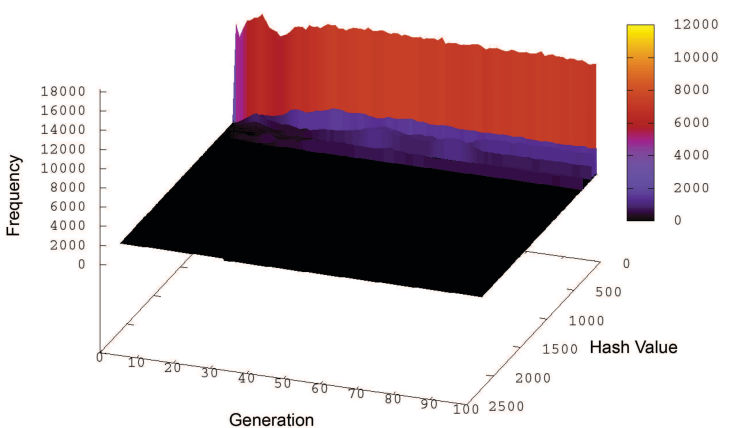

(b)

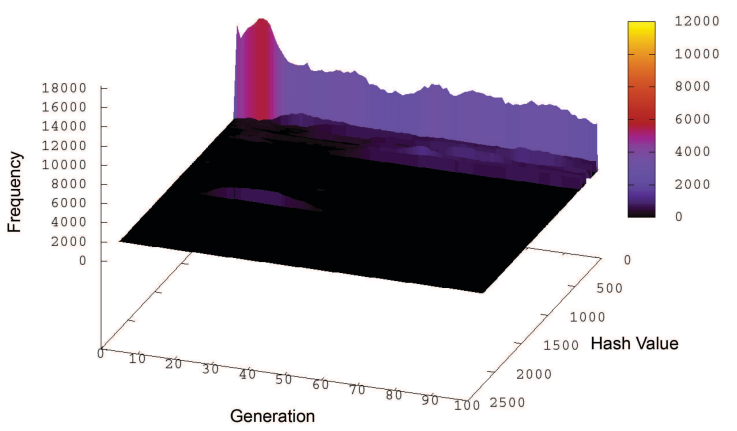

(d)

Figure 6.13: A sampling of 3D plots (run (a) 5, (b) 9, (c) 14 and (d) 29) of fixed-depth subtrees of depth 1, using FST-Hashing, simplification every generation 


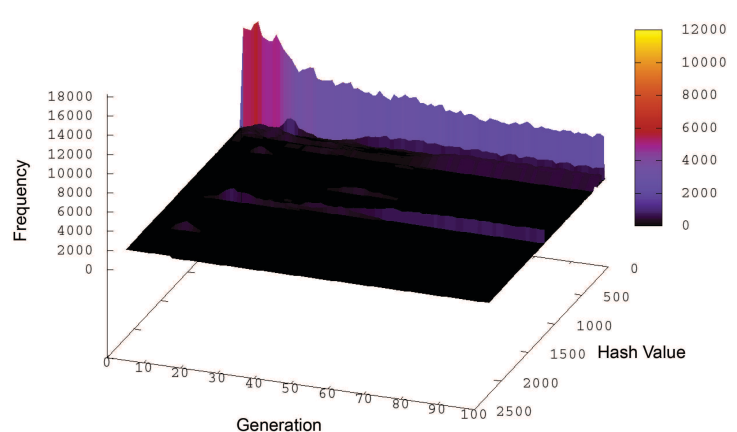

(a)

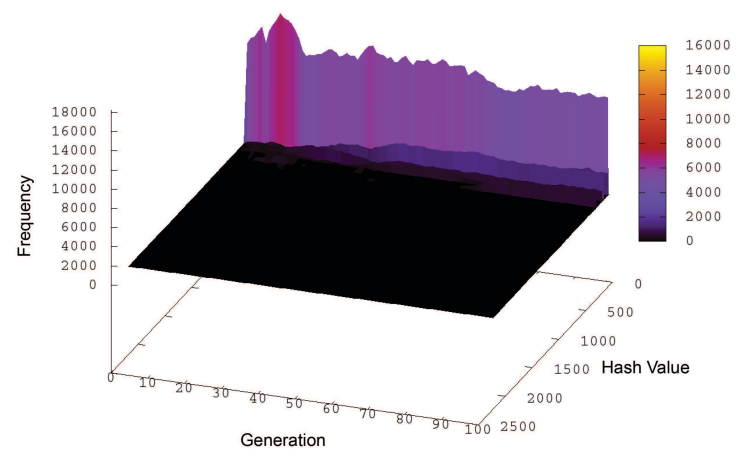

(c)

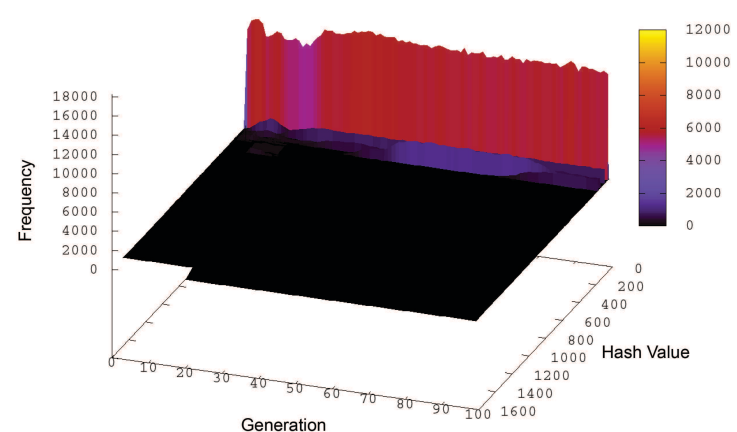

(b)

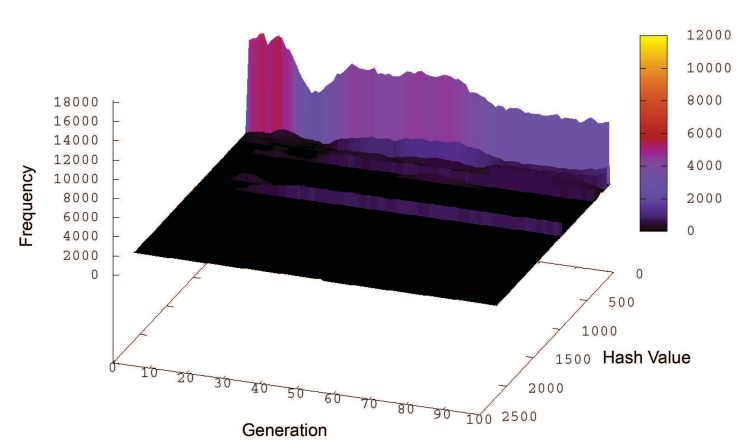

(d)

Figure 6.14: A sampling of 3D plots (run (a) 5, (b) 9, (c) 14 and (d) 29) of fixed-depth subtrees of depth 1, using FST-Hashing, simplification every 5 generations 


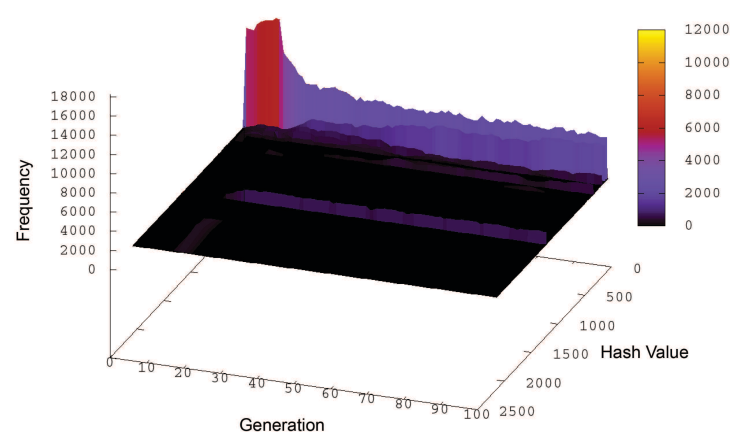

(a)

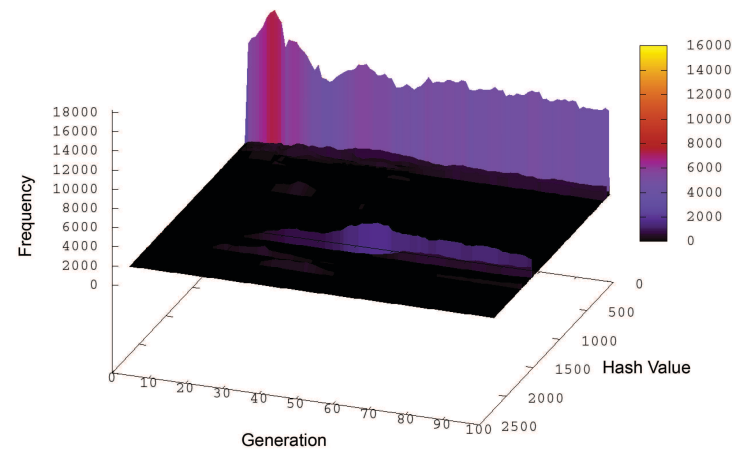

(c)

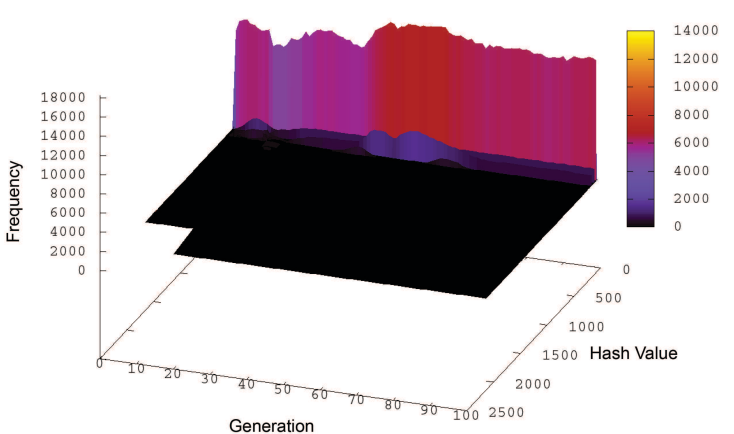

(b)

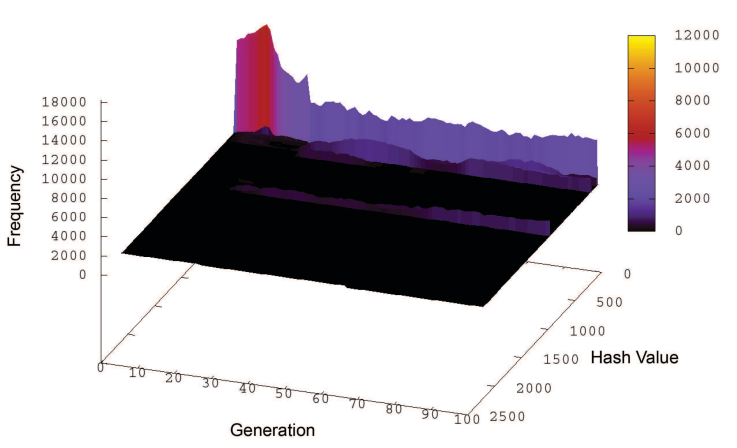

(d)

Figure 6.15: A sampling of 3D plots (run (a) 5, (b) 9, (c) 14 and (d) 29) of fixed-depth subtrees of depth 1, using FST-Hashing, simplification every 10 generations 
new building blocks being created outside the range that not using simplification is restricted to. These plots have a much larger range of FST-Hash values plotted. We can see many of these newly created building blocks as new peaks at various stages of the GP run plots. These peaks suggest that these subtrees (which do not exist in the standard GP runs) exist in the GP population in significant amounts, showing that they are being propagated by the GP system. These findings confirm our results in chapter 5, where (at least at a singular node level), using simplification creates new potential building blocks in the GP populations.

\section{Analysis of Fixed-Subtrees of Depth 2 and 3}

Now we look at some of the bigger fixed-depth subtrees within the populations of individual GP runs. Figures 6.16, 6.17, 6.18 and 6.19 each show plots for the same run (run 5, 9, 14 or 29) when using each of the four simplification frequencies (none, every 1 , every 5 and every 10). Note that this is different from our analysis of depth 1 subtrees, where each figure displayed all four runs for a particular frequency. Using this plot alignment allows us to compare the effects of the four systems when given the same initial conditions.

It is clear that in each of the four displayed runs, using simplification results in different distributions of subtrees in the GP populations. This is not surprising, as even small changes to any one GP generation has the possibility to radically change the direction of that GP run. Interestingly, as we can see in runs 9 and 14 (figures 6.17 and 6.18), the standard GP system has a higher tendency to populate some of the larger subtrees, particularly later in the GP run. This observation could be one of the factors that contribute to program bloat. On the other hand, the systems using simplification do not appear to highly populate these larger subtrees.

This can be further seen when looking at tables 6.4 and 6.5. These two tables show the five most populous subtrees (in the same manner as table 6.3) for subtrees of depth 2 and depth 3 respectively. We can see that 


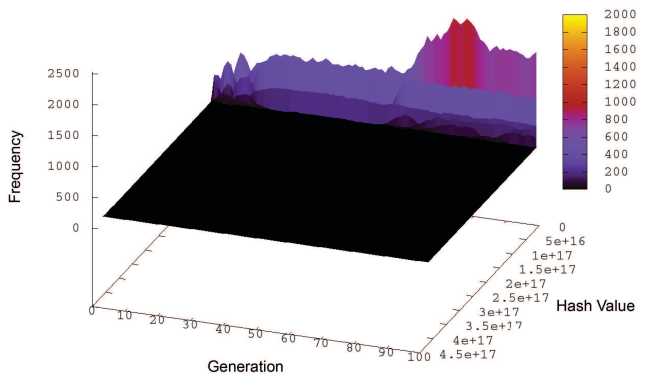

(a)

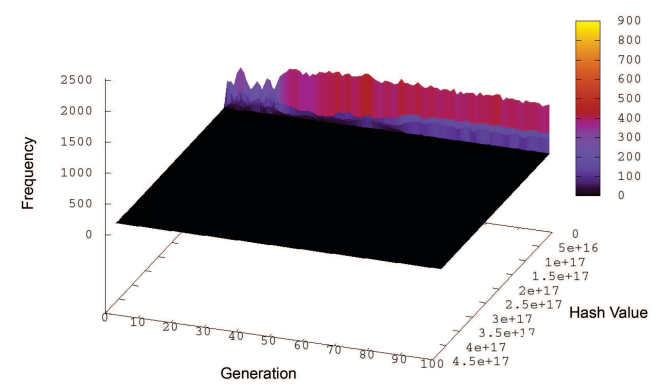

(c)

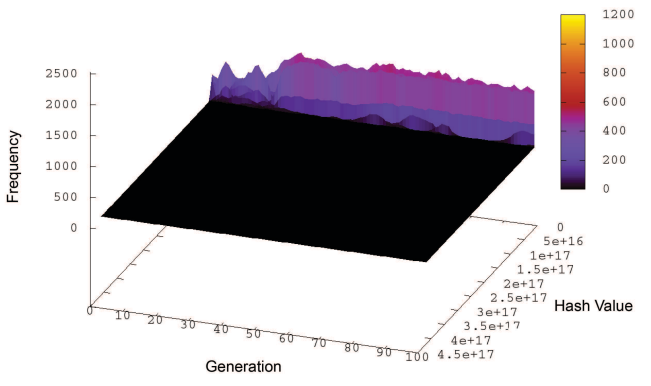

(b)

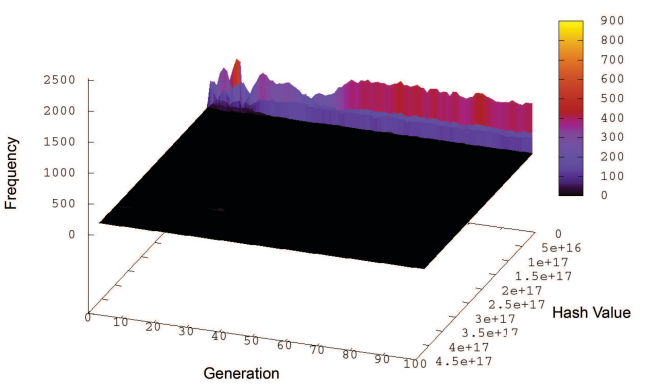

(d)

Figure 6.16: 3D plots of fixed-depth subtrees of depth 2 and 3 for each of the four systems ((a) Without, (b) Every 1, (c) Every 5, (d) Every 10) for run 5 . 


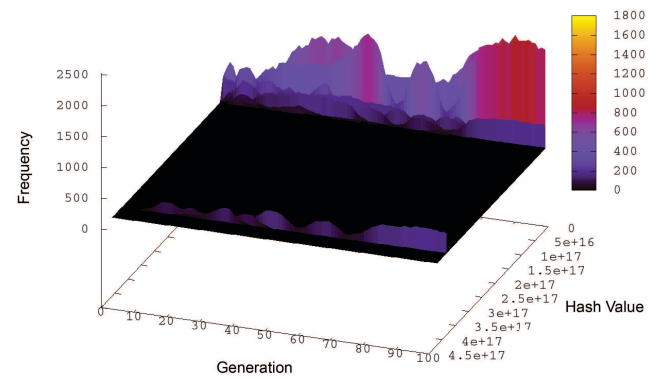

(a)

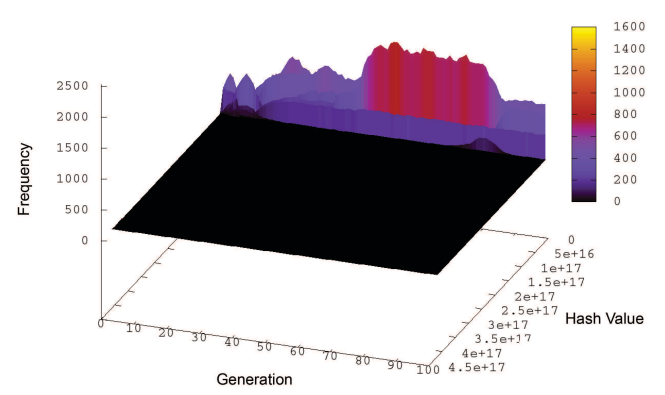

(c)

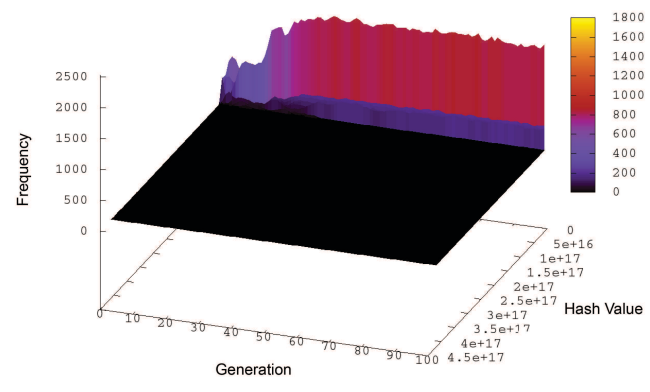

(b)

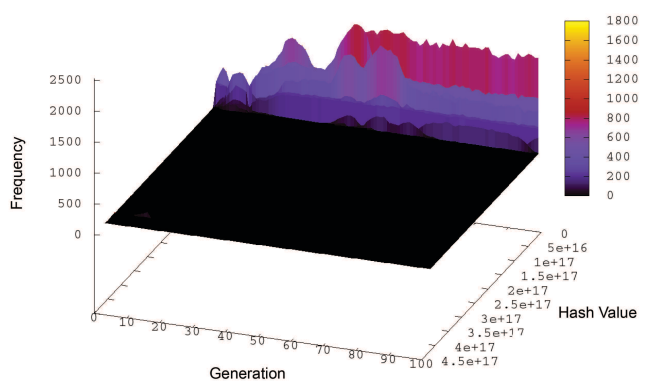

(d)

Figure 6.17: 3D plots of fixed-depth subtrees of depth 2 and 3 for each of the four systems ((a) Without, (b) Every 1, (c) Every 5, (d) Every 10) for run 9. 


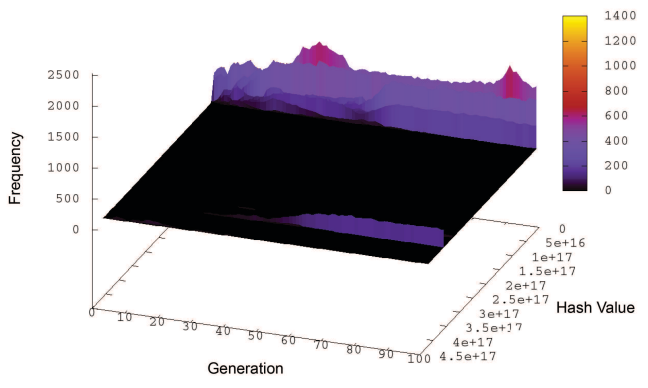

(a)

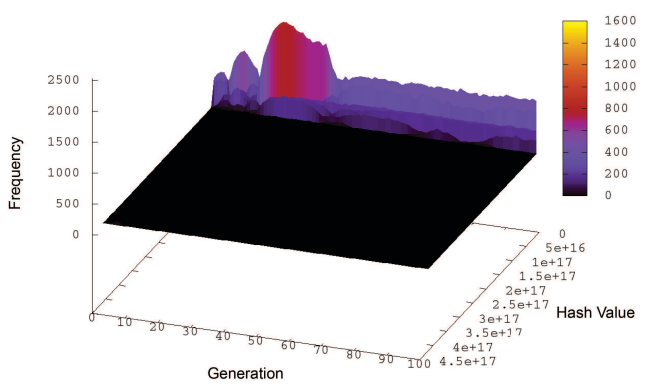

(c)

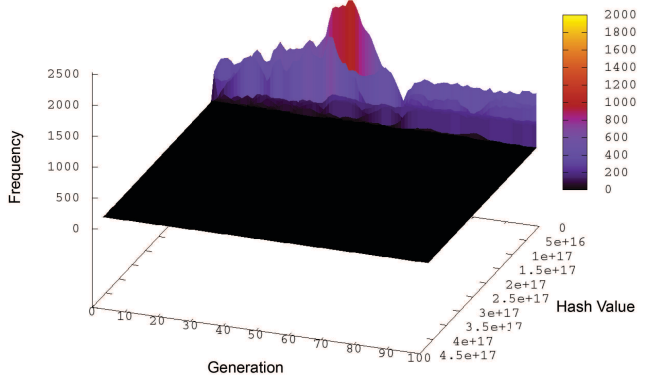

(b)

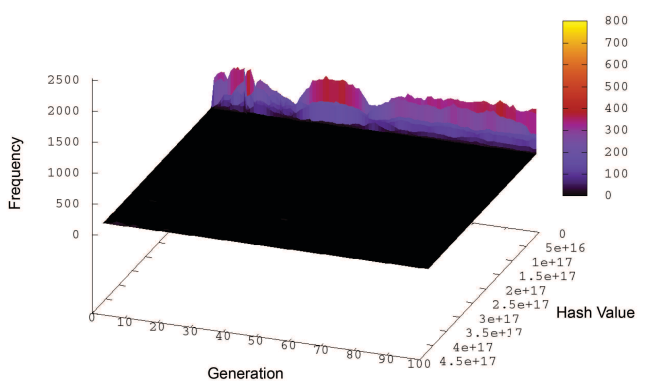

(d)

Figure 6.18: 3D plots of fixed-depth subtrees of depth 2 and 3 for each of the four systems ((a) Without, (b) Every 1, (c) Every 5, (d) Every 10) for run 14. 


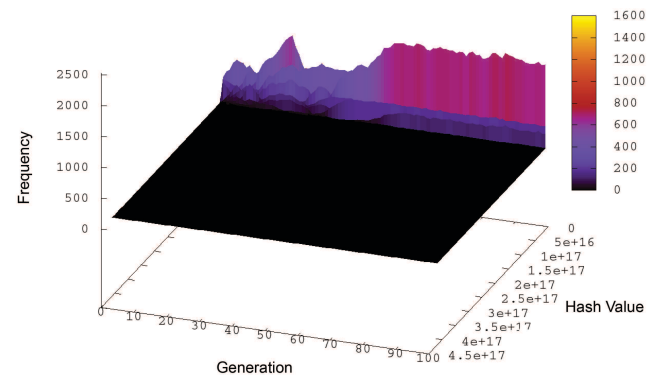

(a)

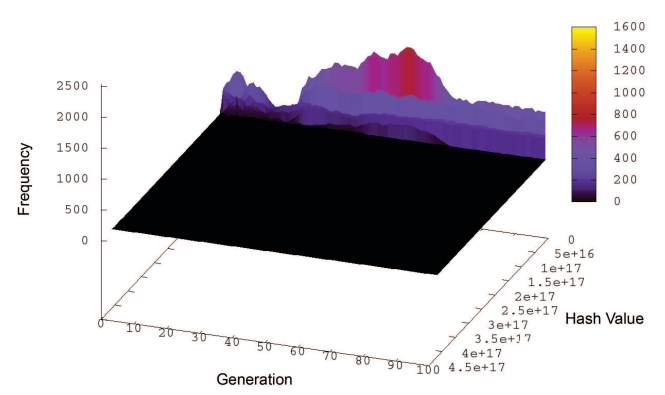

(c)

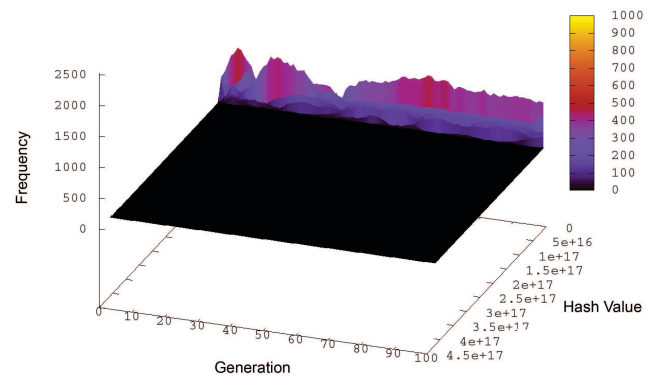

(b)

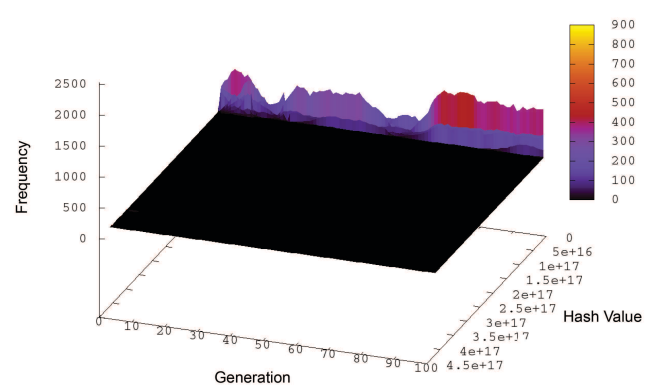

(d)

Figure 6.19: 3D plots of fixed-depth subtrees of depth 2 and 3 for each of the four systems ((a) Without, (b) Every 1, (c) Every 5, (d) Every 10) for run 29. 
Table 6.4: Top 5 Most Popular Subtrees for Depth 2

\begin{tabular}{|cc|cc|cc|cc|}
\hline \multicolumn{2}{|c|}{ No Simplification } & \multicolumn{2}{|c|}{ Every 1 } & \multicolumn{2}{|c|}{ Every 5 } & \multicolumn{2}{c|}{ Every 10 } \\
Hash Value & Freq. & Hash Value & Freq. & Hash Value & Freq. & Hash Value & Freq. \\
\hline 705428 & 1916 & 2474 & 1952 & 2474 & 1514 & 2474 & 1282 \\
136802345 & 1782 & 2474 & 1690 & 565454 & 1382 & 2474 & 2512 \\
148297505 & 1686 & 654802 & 1552 & 2474 & 1826 & 607559 & 1672 \\
2474 & 2160 & 705390 & 1730 & 705510 & 1558 & 705499 & 1092 \\
114385549 & 1758 & 607739 & 1386 & 507254 & 1500 & 705517 & 878 \\
\hline
\end{tabular}

for the no simplification case for depth 2 subtrees that 136802345, 148297505 and 114385549 are among the top five hash values, while in the case for depth 3 we have 6932904075114 and 1788791042021640 . These are larger subtrees than those that occur in the top five for the systems using simplification. Translating these back into subtrees shows that these larger subtrees are usually for manipulating numeric terminals (adding, multiplying and dividing them). This is because the nature of the symbolic regression problem places emphasis on constructing the correct numeric constants, so subtrees like these are important when not using simplification. However, in the systems using simplification these subtrees are often not highly populated. In fact, if we look at plot (a) in figures 6.17 and 6.18, we can see that some large subtree(s) are quite highly populated, while in the systems using GP (plots (b), (c) and (d) of figures 6.17 and 6.18) these subtree(s) are not present. Additionally, in runs 5 and 29 (figures 6.16 and 6.19) no larger subtrees are heavily propagated by the systems using simplification either.

This is likely due to new constants being constructed by the simplification process, which eliminates these types of numeric manipulation subtrees. Because these larger subtrees are highly populated and important to the standard GP runs, we can see that simplification does indeed remove/disrupt building blocks used by the standard GP. This confirms our suspicions from our numerical-node work in chapter 5.

However, there appear to be subtrees that are common to all systems regardless if simplification is used or not. In table 6.4 we can see that hash 
Table 6.5: Top 5 Most Popular Subtrees for Depth 3

\begin{tabular}{|cc|cc|cc|cc|}
\hline \multicolumn{2}{|c|}{ No Simplification } & \multicolumn{2}{|c|}{ Every 1 } & \multicolumn{2}{c|}{ Every 5 } & \multicolumn{2}{c|}{ Every 10 } \\
Hash Value & Freq. & Hash Value & Freq. & Hash Value & Freq. & Hash Value & Freq. \\
\hline 158658268 & 423 & 26041192882 & 402 & 35197473600 & 404 & 31969523949 & 403 \\
6932904075114 & 424 & 35197340444 & 440 & 33558954766 & 432 & 33505039141 & 431 \\
1788791042021640 & 421 & 35198233788 & 496 & 35370472559 & 412 & 30048419759 & 599 \\
30773567274 & 392 & 28538701781 & 390 & 35027761799 & 338 & 155679013 & 396 \\
155679013 & 447 & 159317114 & 391 & 26316073134 & 381 & 35026629553 & 369 \\
\hline
\end{tabular}

value 2474 features prominently in each of the four systems, meaning that it is an important subtree for solving the problem. Indeed, if we translate this hash value back into a subtree, we get: $2474=$ aee $\rightarrow(+f 0$ f0 $)$, which is the simple sum of two feature terminals. Additionally, hash values of the form 705xxx ( $x$ being a wildcard) appear in all four systems as well. All of these subtrees are of the form $d e 0 x x$, which translates to ( $\% \mathrm{f} 0$ $0 . \mathrm{xx})$. This is another form of GP trying to construct the $11 x$ portion of the symbolic regression problem, and it appears that a lot of the subtrees propogated in the GP population are dedicated to tackling that part of the problem. These subtrees show that with or without simplification, similar subtree constructs are propagated through the GP populations in all four systems. In the case of subtrees of depth 3 (table 6.5), there are fewer similarities, although many of the subtrees shared are in the $3 x x x x x x x x x x$ range.

By having so many shared subtrees feature highly in both the standard GP and the systems using simplification, the results show that many important building blocks survive the simplification process and are not removed from the GP populations. Because these important blocks remain, the systems using simplification are not placed at a disadvantage in finding solutions to the symbolic regression problem. 


\subsubsection{Totaling Disrupted and Created Subtrees}

So far we have been analysing individual GP runs, looking for trends in specific runs that might suggest that using simplification can affect the effectiveness of these runs, or disrupt building blocks that would otherwise propagate if no simplification was used. In this subsection we provide a higher level analysis, looking at particular measures that may help us determine the effects of using simplification on GP.

For each generation $\left(g_{i}\right)$ (not including the first generation, where $e v$ ery subtree is new), we can scan through the whole population of subtrees present in the generation and the previous generation $\left(g_{i-1}\right)$ to determine: the subtrees that are present in $g_{i}$ that were not present in $g_{i-1}$; and the subtrees that were present in $g_{i-1}$ that are no longer present in $g_{i}$. By measuring these two aspects, we can get a picture of the disruptive and constructive effects shown in the GP population and will help show whether using simplification results in larger amounts of subtree disruption or construction than the standard GP.

Figures $6.20,6.21$ and 6.22 display graphs when using each of the different simplification frequencies (none, every 1, every 5 and every 10) on the Regression ${ }_{4}$, Regression $_{2}$ and Coins Classification tasks respectively. The blue line represents the number of new subtrees created in each GP generation. The red line represents the number of subtrees disrupted in the each GP generation. The purple line represents the net effect of these two lines, that is the number of subtrees created minus the number of subtrees disrupted. These graphs are created by averaging these values for each generation over all 50 GP runs. Note that the number of subtrees disrupted (red line) has been treated as a negative number (e.g. if there were 100 disrupted subtrees, the plotted value is -100) in order to make the plots clearer and avoid overlapping lines. Additionally, a black horizontal line at $y=0$ has been drawn on each graph. This allows us to easily judge whether there is a net creation or disruption of subtrees overall.

In all of the tasks, there appears to be an overall disruption trend (sig- 


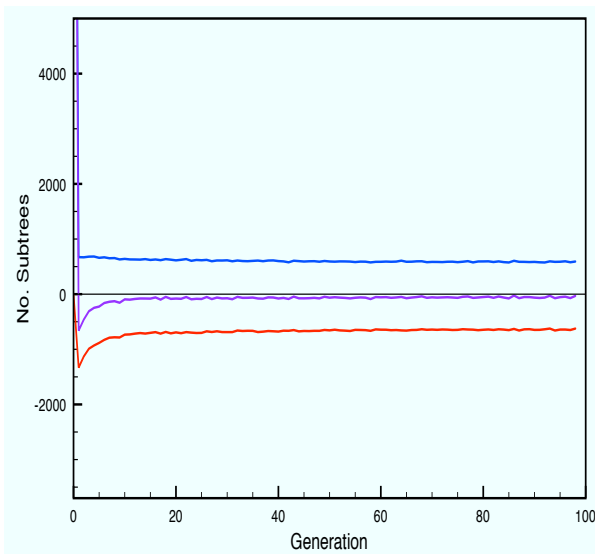

(a) Without

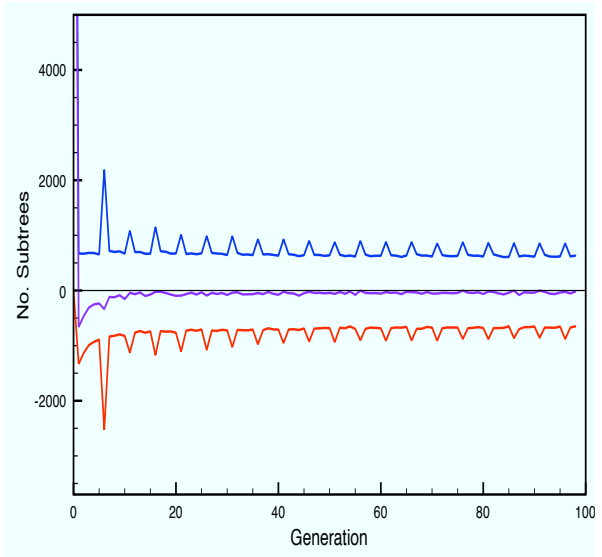

(c) Every 5

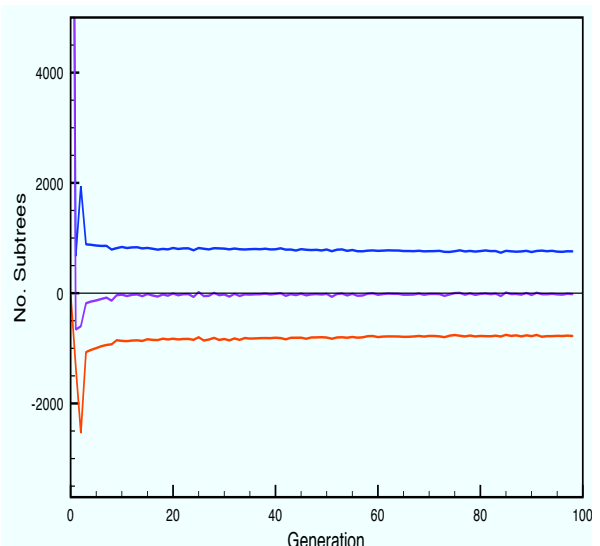

(b) Every 1

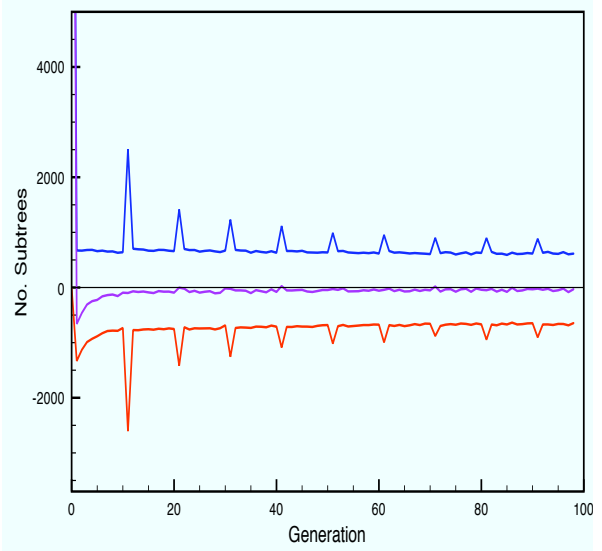

(d) Every 10

Figure 6.20: Number of subtrees disrupted and created during GP evolution of Regression 4 problem, averaged over 50 runs. Results are for (a) No Simplification, (b) Simplification every generation, (c) Every 5 generations and (d) Every 10 generations.

naled by the purple net effect line being below zero). This means that even without simplification, there is a slight bias towards fewer subtrees in the GP population. This can be expected, as the major purpose of GP is to use its form of natural selection to refine the population toward solving a 

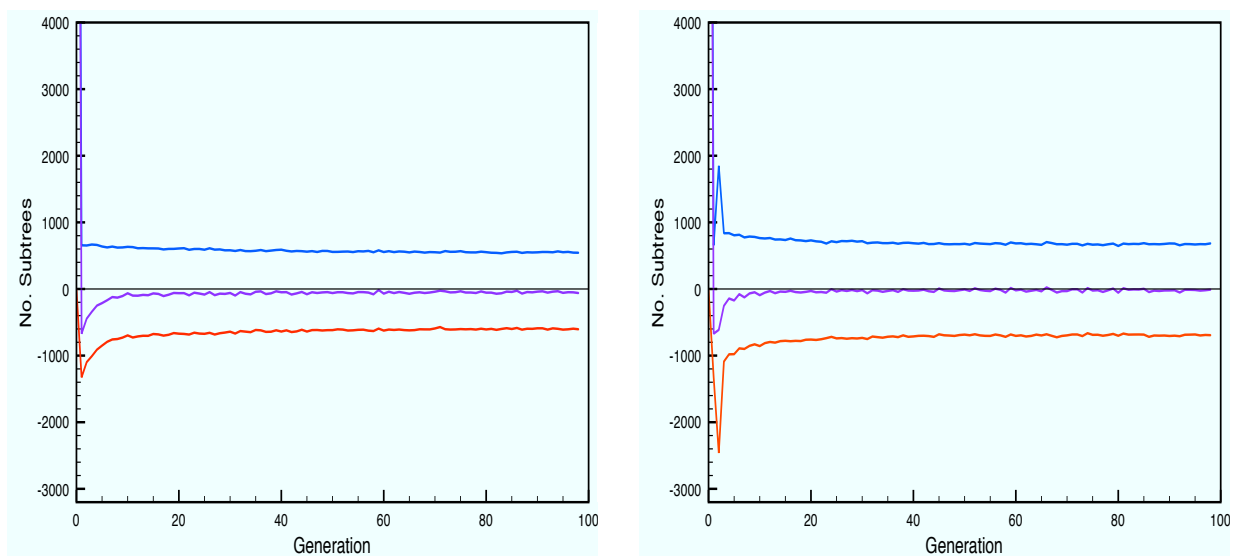

(a) Without

(b) Every 1
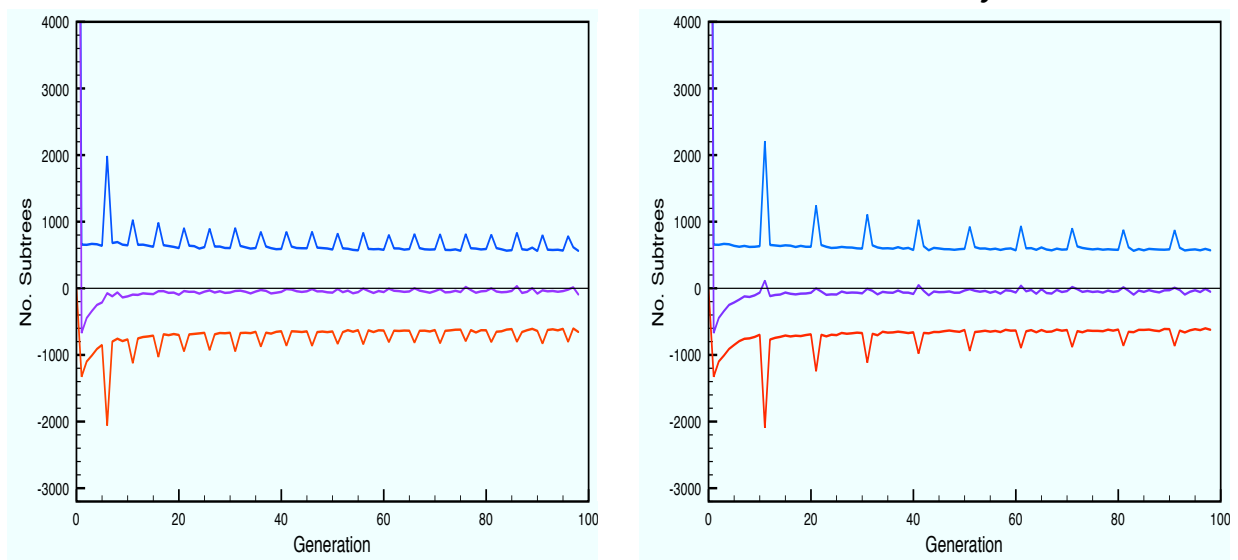

(c) Every 5

(d) Every 10

Figure 6.21: Number of subtrees disrupted and created during GP evolution of Regression 2 problem, averaged over 50 runs. Results are for (a) No Simplification, (b) Simplification every generation, (c) Every 5 generations and (d) Every 10 generations.

particular problem.

Firstly, we compare the graphs for systems using simplification $(b, c$ and d) with the standard GP (a) in each task. In every case, we can see that the red line is "lower" and the blue line is higher when using sim- 


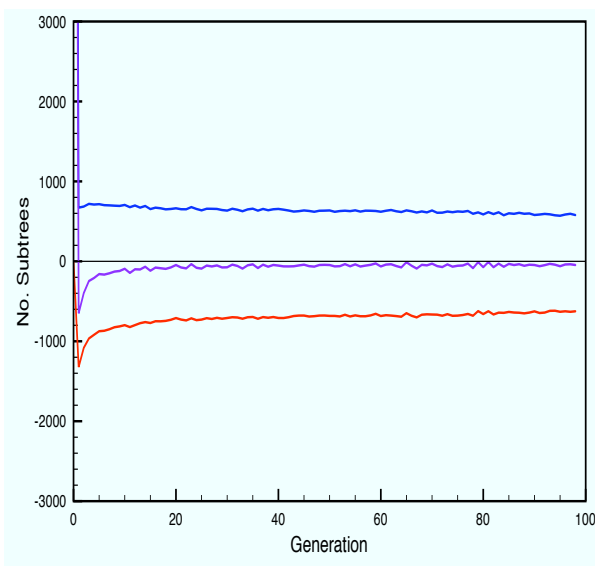

(a) Without

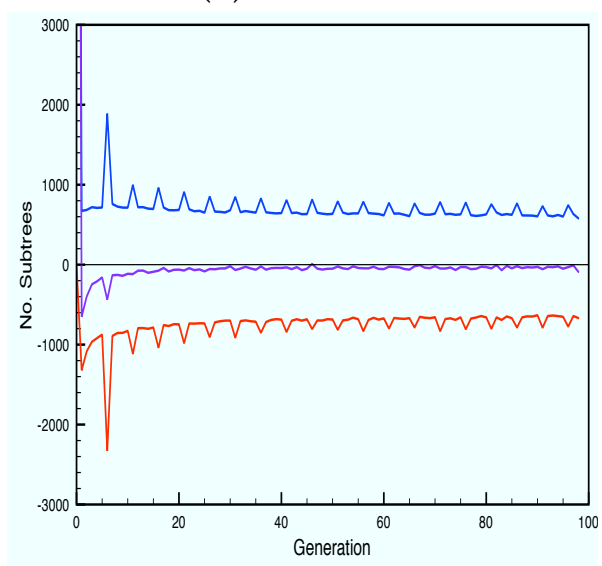

(c) Every 5

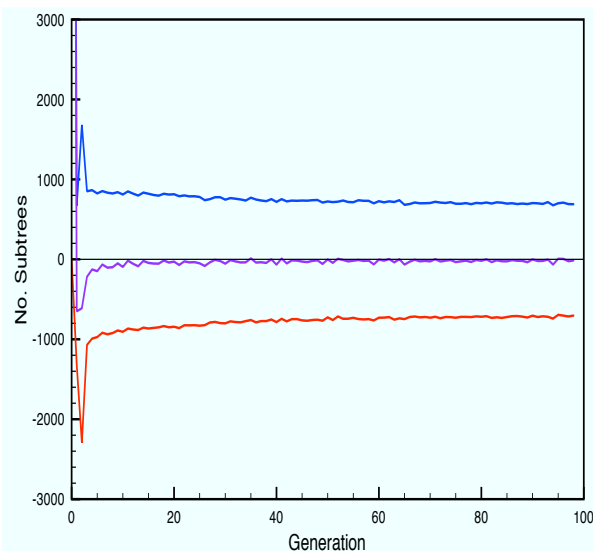

(b) Every 1

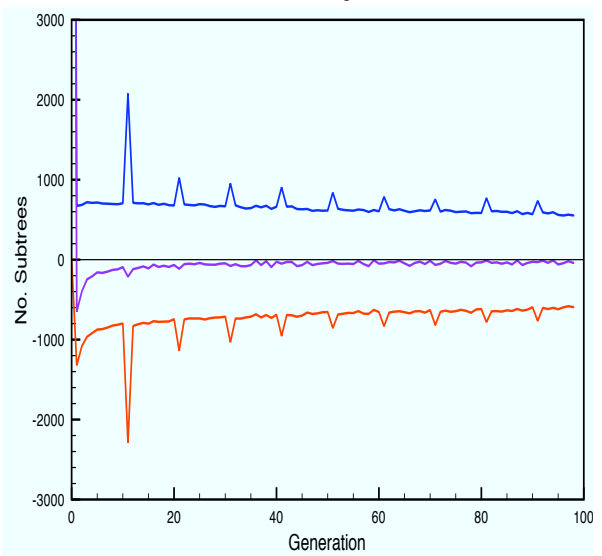

(d) Every 10

Figure 6.22: Number of subtrees disrupted and created during GP evolution of Coins Classification problem, averaged over 50 runs. Results are for (a) No Simplification, (b) Simplification every generation, (c) Every 5 generations and (d) Every 10 generations.

plification. This suggests that by using simplification, we are increasing both the rates of subtree creation and subtree disruption. Furthermore it appears that applying simplification at every generation has increased disruption and creation over using simplification frequencies of 5 and 10. 
Additionally, in the graphs (c and d) for each task we observe a "spiking" phenomenon in the amount of subtree creation and subtree disruption coinciding with any generation in which simplification has been applied. We could expect some "spiking" to occur in the plots for (b) in each task where simplification is applied at every generation. However, when using simplification in all generations, there is no "build-up" of genetic materials and redundancies. This results in no "spiking" trend in the plots for these systems. These results confirm to us that it is the act of simplification itself that is causing the increase in both subtree creation and subtree disruption.

Note that by having increased creation, we are having more new genetic materials added to the GP populations. By having increased disruption, we are losing more of the existing genetic materials that were in the GP populations. Therefore, by having an increase in both creation and disruption, there is a larger overall change in the genetic content of the current generation compared with the previous generation when using simplification. We can call the larger gap between the creation and disruption lines as having a larger genetic turnover.

With an increased genetic diversity, one may suppose that using simplification should result in better programs being evolved, as it can search more of the GP search space concurrently. However, if we look at each graph again, even though the genetic turnover is different in each of the four systems when performing each task, the overall net effects (purple line) are very similar. Our results from tables 6.3, 6.4 and 6.5 show that very similar subtrees become highly populated, whether simplification is used or not. It could be that the majority of those subtrees being disrupted and created are at the extremities of the GP population and do not strongly affect the GP system. This may be one of the reasons that using simplification has been shown to have no significant effect (positive or negative) on the fitness of solution programs produced by the GP, and can even result in slightly better performance in many cases. 


\subsection{Chapter Summary}

In this chapter, we performed further analysis of the effects of using program simplification on the internal "building blocks" of GP populations. We built upon the numerical-node model we used in chapter 5 and used a more complex fixed-depth subtree model. This model consisted of using subtrees of a specified fixed depth.

In order to properly track and visualise these subtrees, we developed FST-Hash, a hashing method to convert these subtrees into numeric values for plotting into graphs for visualisation. FST-Hash is a two step process, the first step consisting of translating the prefix ordering of the standard GP tree program into a level ordering. The second step translated each node into a corresponding enumerated digit (in our case, the values $0-$ 9 and $a-e)$ and converting the resulting numeric sequence into a base10 (decimal) number for plotting. In this way, we could visually track subtrees as they were populated and desolated through each individual GP run.

Using FST-Hash, we analysed the same Regression 4 problem that we used for the numerical-node model in chapter 5. Analysis of the subtrees of depth 1 confirmed our results from chapter 5 , where using simplification was able to create new building blocks outside of the range that the standard GP is restricted to. Our focus then turned to the larger subtrees (depth 2 and 3). We found that using simplification meant that some of the larger highly populated subtrees that are in the standard GP runs are no longer highly populated in GP runs for the systems using simplification. This suggests that the simplification process does disrupt building blocks which are important to the standard GP, although these larger subtrees may often be subtrees that simply manipulate numerical-nodes. However, we also found that many building blocks were commonly found in both the systems using simplification and the standard GP, showing that many important subtrees/building blocks are retained. 
We then tracked the number of subtrees being disrupted and created from generation to generation and averaged these over the whole 50 runs for each task. Through this analysis, we determined that using simplification at any frequency leads to an increase in both the amount of subtrees being disrupted and created in each generation, over the standard GP's "natural" levels. While this higher genetic turnover could lead to higher amounts of genetic diversity, the overall net effect (subtrees created - subtrees disrupted) was relatively consistent between the standard GP and the systems using simplification.

Our findings help explain why using simplification does not appear to have any significant effect on the fitness of solution programs found by GP, despite having better population diversity. 


\section{Chapter 7}

\section{Reducing Costs of Fitness Evaluations}

\subsection{Introduction}

In the previous chapters, we have described a program simplification approach to removing redundancy from evolved GP programs during evolution and analysed how this program simplification affects the building blocks within GP populations. This online program simplification approach provides a method of reducing the computational cost of GP as well as reducing the program size. In this chapter, we will describe a different approach to reducing computational costs in GP, this time by considering fitness evaluations.

Fitness Evaluation takes up a significant proportion of the computation performed for GP (and EC in general). This is because every individual of a population in every generation needs to be evaluated for all the given training fitness cases. While several methods have been investigated to reduce the cost of performing fitness evaluation, most of these previous works have not been applied to GP (see chapter 2). However, none of the GP methods exploit the fact that many subtrees in GP programs are functionally or algebraically equivalent. In this chapter, we will look at a couple 
of methods. The first will look at avoiding re-evaluation of programs reproduced by elitism. The second method will be based on subtree caching, while also taking advantage of the algebraic equivalence of many of these subtrees.

\subsubsection{Chapter Goals}

This chapter aims to look at two different methods of reducing the number of node evaluations required to evaluate GP programs. Both of these methods try to avoid performing fitness evaluations, while maintaining the effectiveness of the GP programs evolved. More specifically, this chapter will look to do the following:

- modify the standard GP to avoid re-evaluating programs produced using elitism reproduction; determine whether this modification can significantly reduce the amount of CPU time required for GP to run, when compared to the standard GP;

- develop a new method of subtree caching, utilising algebraic equivalence techniques; determine whether this method can significantly reduce the node evaluation cost without decreasing the effectiveness of the programs produced, compared with the standard GP approach;

- determine how choice in the size of subtrees that are cached affects the performance of GP systems using the subtree caching method; and

- investigate how the number of fitness cases in a given GP task can affect the effectiveness of subtree caching. 


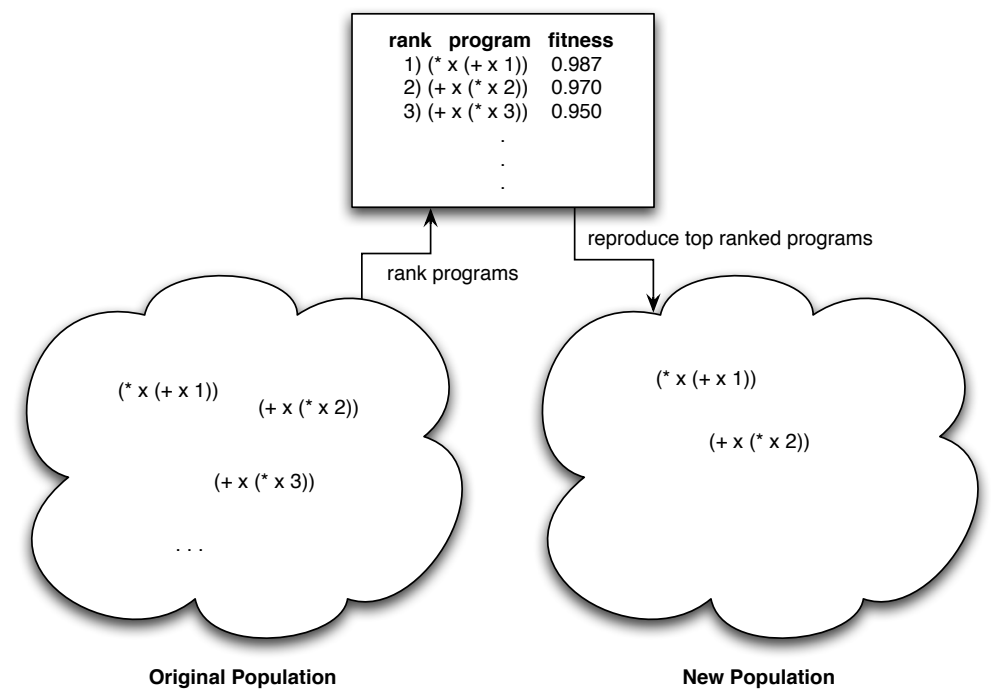

Figure 7.1: How elitism reproduction is performed.

\subsection{Avoiding Re-evaluation of Reproduced Pro- grams}

Many GP systems employ some form of reproduction as a way of guaranteeing (or providing a high chance) that the next GP generation is at least as fit as the current generation. One such method is elitism.

Elitism simply takes a ranked order of the population (in terms of fitness) and copies a specified number of the highest ranked (fittest) programs directly into the next generation. This process is illustrated in figure 7.1.

In the standard GP, during the fitness evaluation stage, all programs in the GP generation are evaluated. In the cases for programs copied over by elitism, this is clearly superfluous effort, as the fitness of the copied program has not changed since the last generation. In past experiments, we have been using an elitism rate of $10 \%$ (a common value for reproduction rate) with a GP population of 500. This translates to 50 GP programs that are being unnecessarily re-evaluated in fitness. By avoiding 
the re-evaluation of these programs we reduce the overall number of node evaluations that are being performed and can easily reduce the amount of computation used in the GP run.

We made a simple modification to the GP system to avoid re-evaluating any GP program which was propagated to the new generation through elitism reproduction. Because this modification is so basic, there is essentially no overhead added to the GP system. Since all we are doing is copying a past evaluation result for a reproduced program, there are no side effects that can lead to an incorrect evaluation for a program. Thus, the GP programs evolved in the standard GP system and the modified system (when using the same initial conditions and random seed) are identical. Therefore the effectiveness of the programs being produced by both systems are also identical.

\subsubsection{Experiments}

We performed experiments on six GP tasks using the newly modified system and compared the results with the standard GP system. The six tasks chosen were: Regression 2 , Regression 3 , Shapes, Coins, Breast Cancer Wisconsin $(\mathrm{BCW})$ and the SPECT dataset. For more information on these datasets, consult chapter 3 .

Figures 7.2, 7.3 and 7.4 show a comparison of the standard GP system and a GP system using elitism re-evaluation avoidance. Each graph shows the CPU time taken by each system when using different rates of elitism (we used rates of between $0 \%$ and $100 \%$, in 10\% increments). Each datapoint is an average measured CPU time from 50 individual runs. The gray line in each graph represents the performance of the standard GP, while the black line represents the performance of GP using elitism re-evaluation avoidance.

The graphs clearly show that using elitism re-evaluation avoidance results in a lower amount of CPU time required to complete runs. As ex- 


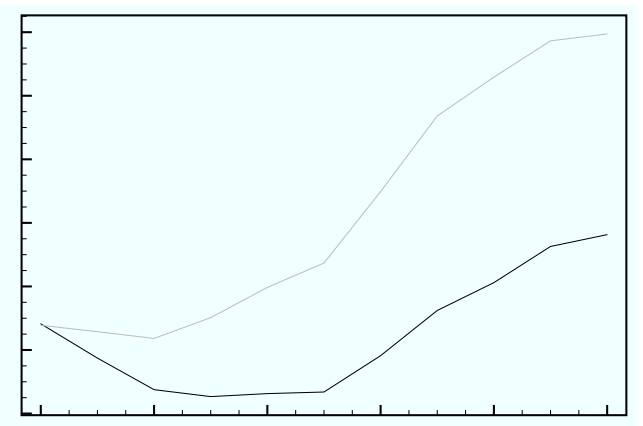

Regression $_{2}$

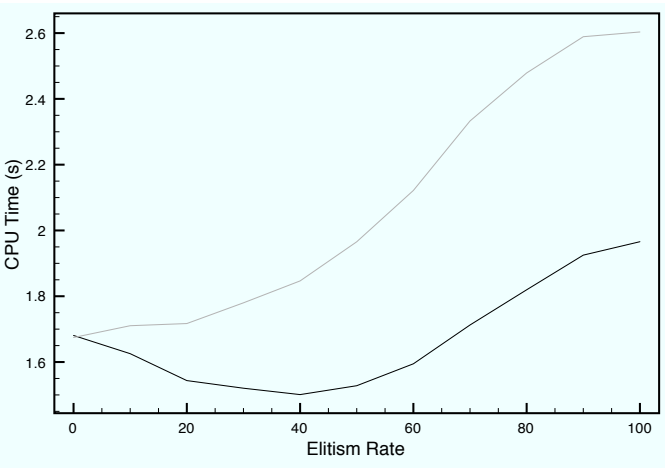

Regression $_{3}$

Figure 7.2: Elitism Re-evaluation Avoidance: Symbolic Regression Tasks

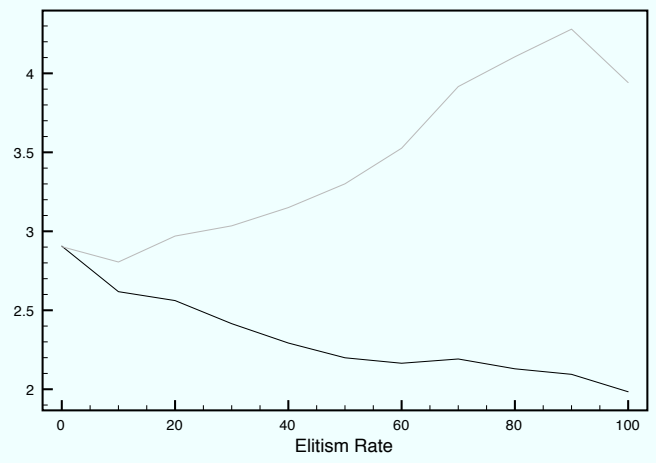

Shapes

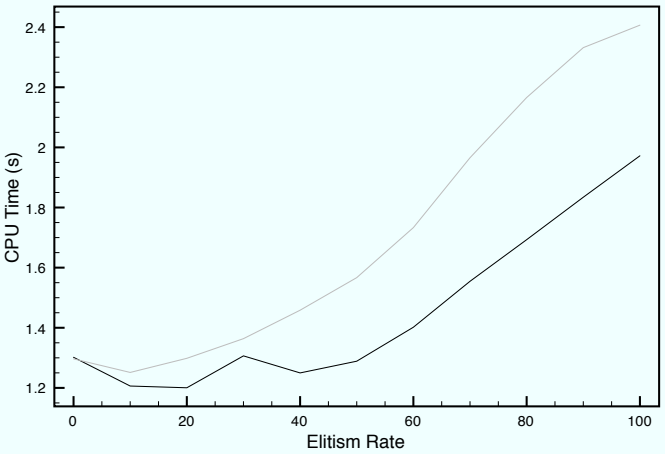

Coins

Figure 7.3: Elitism Re-evaluation Avoidance: Image Classification Tasks

pected, as the elitism rate was increased in each task, the gap between the CPU time taken by the standard GP and that taken by the GP system using avoidance widened. Also, the size of the time saving appears to be task specific and each task showed different amounts of savings gained for each task. For example, the SPECT task showed the two systems to be fairly close (at 100\% elitism, having 14\% time saved), while the results for the Shapes task shows a large gap in measured CPU time between the two systems (at 100\% elitism, having 50\% time saved). 


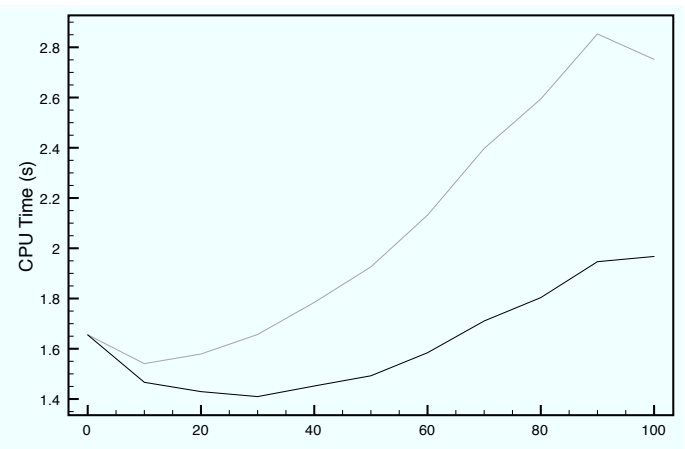

BCW

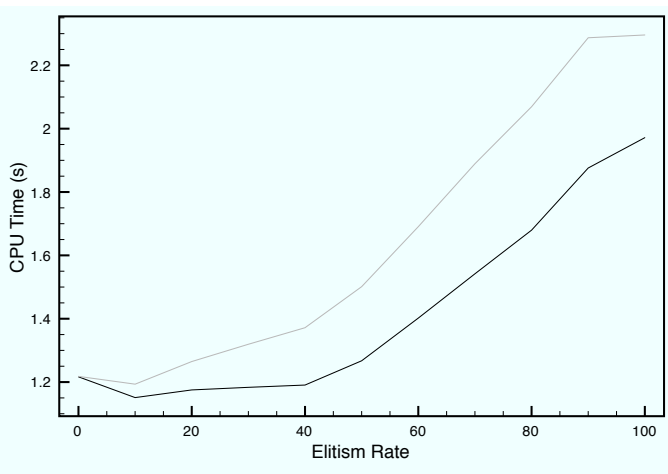

SPECT

Figure 7.4: Elitism Re-evaluation Avoidance: Medical Classification Tasks

Table 7.1: Measure CPU Times for the six tasks, using 10\% elitism

\begin{tabular}{|c|ccc|}
\hline Task & Standard GP & Avoidance & Savings (\%) \\
\hline Regression $_{2}$ & $1.6576 \pm 0.163426$ & $1.5746 \pm 0.152962$ & $5.007 \%$ \\
Regression $_{3}$ & $1.7104 \pm 0.147969$ & $1.6254 \pm 0.140659$ & $4.970 \%$ \\
Shapes & $2.8056 \pm 0.310466$ & $2.6178 \pm 0.280582$ & $6.694 \%$ \\
Coins & $1.2514 \pm 0.166048$ & $1.2062 \pm 0.158486$ & $3.612 \%$ \\
BCW & $1.5408 \pm 0.242678$ & $1.4664 \pm 0.226663$ & $4.829 \%$ \\
SPECT & $1.1934 \pm 0.117231$ & $1.151 \pm 0.112025$ & $3.553 \%$ \\
\hline
\end{tabular}

Elitism is typically set at a low rate, usually around $10 \%$. Table 7.1 shows the CPU times that were obtained when using this elitism rate. It shows that the savings to be expected from using this approach in a "realistic" GP setting may be around $3.5-7 \%$, depending on the problem. Despite the simplicity of this modification to the GP system, it can result in some significant saving in time.

However, this type of avoidance only affects programs that were reproduced using elitism. This means that the elitism rate is essentially an upper limit on how much computation can be saved using this approach (i.e. if fitness evaluation accounted for $100 \%$ of GP computation, then by avoiding evaluation of $10 \%$ of programs we can get at most a $10 \%$ sav- 
ing in computation). In the next section, we will look at an approach that potentially affects all programs in the GP populations, and therefore can potentially lead to much higher amounts of time being saved.

\subsection{Avoiding Re-evaluation by using Subtree Caching}

Different GP programs in the same population often have certain subtrees in common. This results from GP's retention of fit programs using genetic selection, as well as the use of reproduction. Crossover further propagates some subtrees into multiple individuals of a new population. This fact has helped develop ideas of code reuse (e.g. Automatically Defined Functions). We can exploit this repetition through caching in order to save the number of node evaluations that need to be performed, and hence save CPU time.

Despite having a common goal (reducing the resources needed to perform GP evolution), caching differs from our earlier simplification method. The main aim of simplification is to reduce the memory usage of the GP populations, by making the programs smaller and eliminating bloat. Any CPU time reduction obtained is a side-effect of the GP system having to process smaller programs. The main aim of caching on the other hand is to reduce the number of node evaluations and CPU time required for GP to evolve solutions, by exploiting common subtrees amongst the GP population. Caching does not aim to reduce the size of programs within GP at all.

The following subsection explores the number of distinct tree structures that occur when considering different sized trees. This is important for us to consider when designing a subtree caching mechanism. 


\subsubsection{Calculating the number of possible distinct trees of depth $n$}

In order to make a thorough analysis of the caching performance, it is useful to know exactly how many different trees of a certain depth exist, at least to help understand the magnitudes of how much difference there is between caching trees of depth 3 and caching trees of depth 5. Here we assume that trees only have two children, as the function set we have used for most of this thesis only take two child nodes as function parameters.

Firstly we define the base cases (depth of 0,1 ). For depth of 0 the answer is trivial, as there can only be one combination of a single node. Similarly, for depth of 1 , there is only one tree possible (the root node and two children.

$$
\begin{aligned}
& \text { NumTrees }(0)=1 \\
& \text { NumTrees }(1)=1
\end{aligned}
$$

In order to generalise these cases to the $n^{\text {th }}$ case, we can think of the problem in this way. Assume that we know the number of distinct trees for all previous depths 0 through $(n-1)$. To extend this to a depth of $n$, we can just create a new root node at the top of the tree (which increases the depth by 1). Now, for this new root node, we have the following possible situations for making trees of depth $n$ :

1. We place a tree of depth $(n-1)$ as the left child, and then place a smaller tree (depth 0 to depth $(n-2)$ ) as the right child.

2. We place a tree of depth $(n-1)$ as the right child, and then place a smaller tree (depth 0 to depth $(n-2))$ as the left child.

3. We place equally sized trees of depth $(n-1)$ as both the left and right children. 
Since we know the number of distinct trees for the lower depths, we know that each of these three situations yields the following distinct number of trees:

1. The left child has NumTrees $(n-1)$ trees, while the right child can have anywhere from NumTrees $(0)$ to $N u m T r e e s(n-2)$ trees. This gives $N u m \operatorname{Trees}(n-1) \cdot \sum_{i=0}^{n-2} \operatorname{NumTrees}(i)$.

2. This is just the inverted case of above. The right child has NumTrees(n1) trees, while the left child can have anywhere from NumTrees (0) to NumTrees $(n-2)$ trees. This gives $\sum_{i=0}^{n-2} N u m T r e e s(i) \cdot N u m T r e e s(n-$ $1)$.

3. This is the simplest case, for both left and right children, they both can have NumTrees $(n-1)$ different subtrees, so the total number is simply the product of left and right: NumTrees $(n-1) \cdot N u m T r e e s(n-$ $1)$.

Summing these three cases together gives us the total number of distinct trees of depth $n$ (or NumTrees(n)):

$$
\begin{aligned}
\operatorname{NumTrees}(n) & =\operatorname{NumTrees}(n-1) \cdot \sum_{i=0}^{n-2} \operatorname{NumTrees}(i) \\
& +\sum_{i=0}^{n-2} \operatorname{NumTrees}(i) \cdot \operatorname{NumTrees}(n-1) \\
& +\operatorname{NumTrees}(n-1) \cdot \operatorname{NumTrees}(n-1)
\end{aligned}
$$

Which can be simplified by factoring out the NumTrees $(n-1)$, giving the rewritten equation for the general case (equation 7.3):

$\operatorname{NumTrees}(n)=\operatorname{NumTrees}(n-1)\left(\operatorname{NumTrees}(n-1)+2 \cdot \sum_{i=0}^{n-2} \operatorname{NumTrees}(i)\right)$ 
Table 7.2: Number of distinct trees calculated for depths 0 to 6

\begin{tabular}{cc}
\hline Depth $n$ & Distinct Trees NumTrees $(n)$ \\
\hline 0 & 1 \\
1 & 1 \\
2 & $1(1+2 \cdot 1)=3$ \\
3 & $3(3+2 \cdot[1+1])=21$ \\
4 & $21(21+2 \cdot[3+1+1])=651$ \\
5 & $651(651+2 \cdot[21+3+1+1])=457653$ \\
6 & $457653(457653+2 \cdot[651+21+3+1+1])=2.100659306 \times 10^{11}$ \\
\hline
\end{tabular}

Note that these equations so far only give the number of trees which are distinct structurally, without taking into account the different combinations of function nodes and terminal nodes that are possible within the same tree structure. Calculations for the first seven tree depths are given in table 7.2 (a maximum tree depth of 6 is usually used in GP setups).

There is extreme growth in the distinct number of trees even just between depths 4 and 5. We want to use caching in order to avoid reevaluations of subtrees which have been evaluated already in either previous generations or earlier in the current generation. In order to optimise the caching process, and ensure that only "useful" caches are performed, we need to know the probability that a subtree will be required at a later stage. It would appear from our calculations that caching trees larger than depth 4 may result in a large number of false collisions (as it requires an unreasonably large number of distinct hash values in order to avoid such collisions). Furthermore, the chances that subtrees of this size (and larger) that are stored in the cache will be later retrieved is very small. Hence, we may be required to cache subtrees of size 3 or smaller in order to get good caching performance. 


\subsubsection{Subtree Caching using a Hashing for Equivalence MEthod (SCHEME)}

In Subtree Caching, the aim is to reduce the number of node evaluations performed by storing the evaluation values for already evaluated subtrees into the cache. Later evaluations of nodes will then consult the cache before proceeding further to determine whether the current subtree being evaluated has been cached before. If it has, then the evaluation values are fetched from the cache and the fitness evaluation method does not have to travel any further down that program tree branch.

Since SCHEME's fitness evaluation method is applied to all programs during the GP runs, the cache will quickly fill as the GP run progresses. This means that early GP generations are more likely to have more node evaluations than later GP generations. In essence, by caching, we are hoping to only ever have to evaluate a subtree the first time it occurs in the run. Any subsequent existence of the same subtree in any GP generation can then use those evaluated values that were calculated the first time.

\section{The Method}

Now we describe the subtree caching method as a whole.

Figure 7.5 shows pseudo-code for the fitness evaluation method used in the standard GP. The fitness evaluation method recurses down to the bottom of the tree and then builds up the evaluated values for each node. This set of root node evaluation values will be used to determine the programs fitness (e.g. classification accuracy, mean squared error).

SCHEME implements the cache as a hash table, which allows very fast storage and lookup. The entries within the hash table are indexed by algebraic equivalence hashes described earlier in chapter 4. By doing this, multiple subtrees which are not only structurally identical, but also algebraically equivalent subtrees (although they may differ wildly in structure) will consult the same hash entry. 


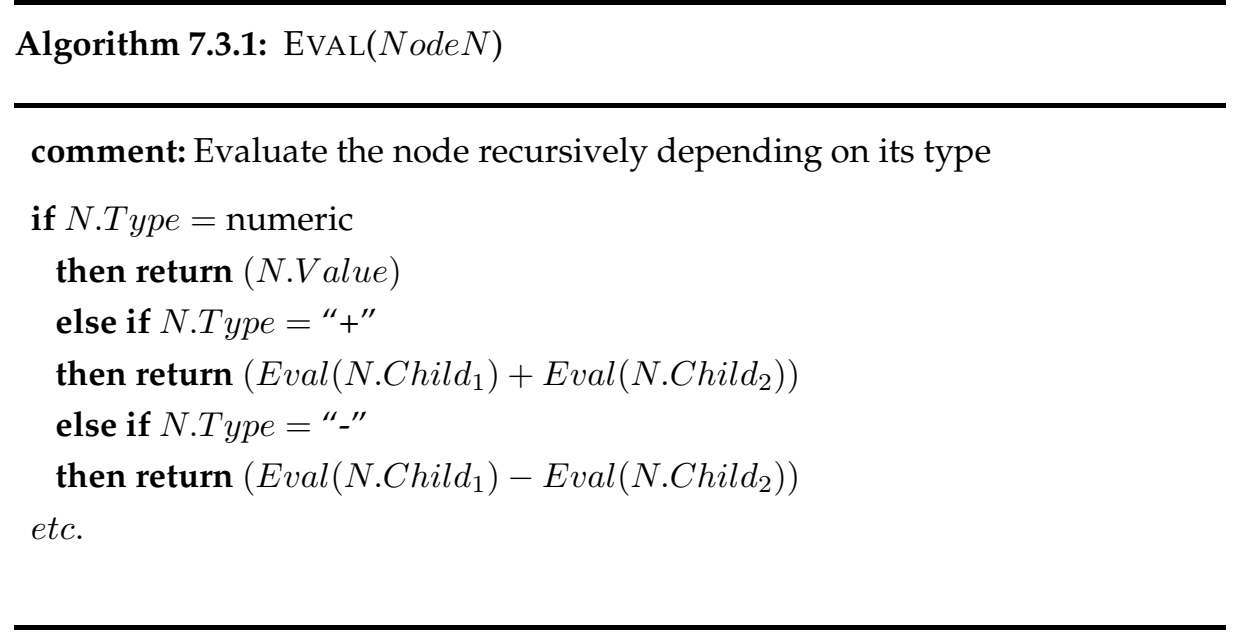

Figure 7.5: Pseudo-code for Standard GP fitness evaluation method.

SCHEME relies on the hash values of subtrees for lookup in the cache, so we need to precompute these hashes before fitness evaluation takes place. The hashing function traverses the program in a bottom-up postfix order. The hash values of terminal nodes are straight forward to calculate, and function nodes are then calculated from the hash values of their children. Although this procedure will add some overhead, it only requires a single parse of the program tree with one set of randomly generated feature terminal values. In this sense, precomputing the algebraic equivalence hash is similar to evaluating a program's fitness using just a single fitness case. Since we are saving on evaluating subtrees on often hundreds (or even thousands) of fitness cases, adding this small amount of overhead is expected to be offset by the large savings caching brings.

Because there is overhead associated with caching, and the fact that a large proportion of subtrees cached may not be used in future generations, it is often undesirable to cache all of the possible subtrees in the GP populations. In SCHEME we control the caching process by defining a caching depth, which determines the depth of subtrees that are eligible for caching. Depth indicates the number of "layers" or "levels" that the 
subtree contains (e.g. a subtree of depth 0 is simply a singular node with no links, while a depth of 1 has a single level of links). Calculating the depth of each node can be done simultaneously with precomputing the algebraic equivalence hash (by using a depth of 0 for a terminal node, and calculating the depth of a function node to be the maximum depth of its children, plus one), and therefore does not require any additional parsing of the program tree. Examples of subtree structures of various depths are given in figure 7.6.

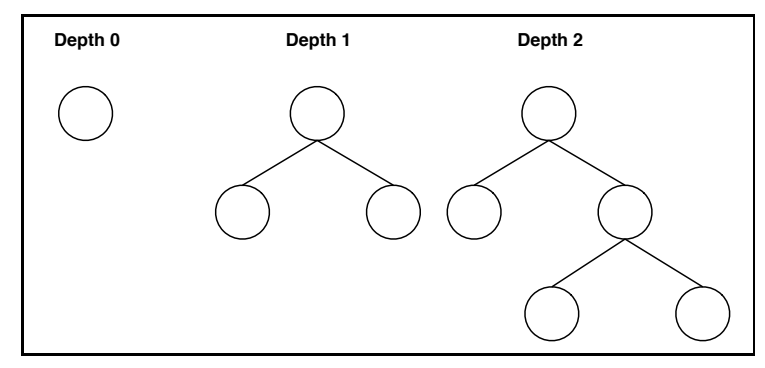

Figure 7.6: Examples of subtrees of various depths.

Figure 7.7 displays pseudo-code for the new fitness evaluation method using SCHEME. As you can see, the first thing the method does is to check whether the subtree extending from the given node is already present in the subtree cache. This is simply done by looking up the node's hash value in the cache. If a match is found (a cache hit), then the evaluation values which have been stored in the cache are returned to the node's parent (and avoiding traversing down the subtree further). If no match is found, then the node needs to be evaluated normally.

After a node has been evaluated (which does not occur if there is a cache hit), then the node's depth is used to determine whether it is eligible for caching. If the depth is not equal to the defined caching depth, then the fitness evaluation continues to the next node. If they are equal, then the evaluation values which have just been calculated are stored into the subtree cache using the node's hash value as the index. As doing this incurs a cost penalty of copying the evaluation values to the cache, this is 


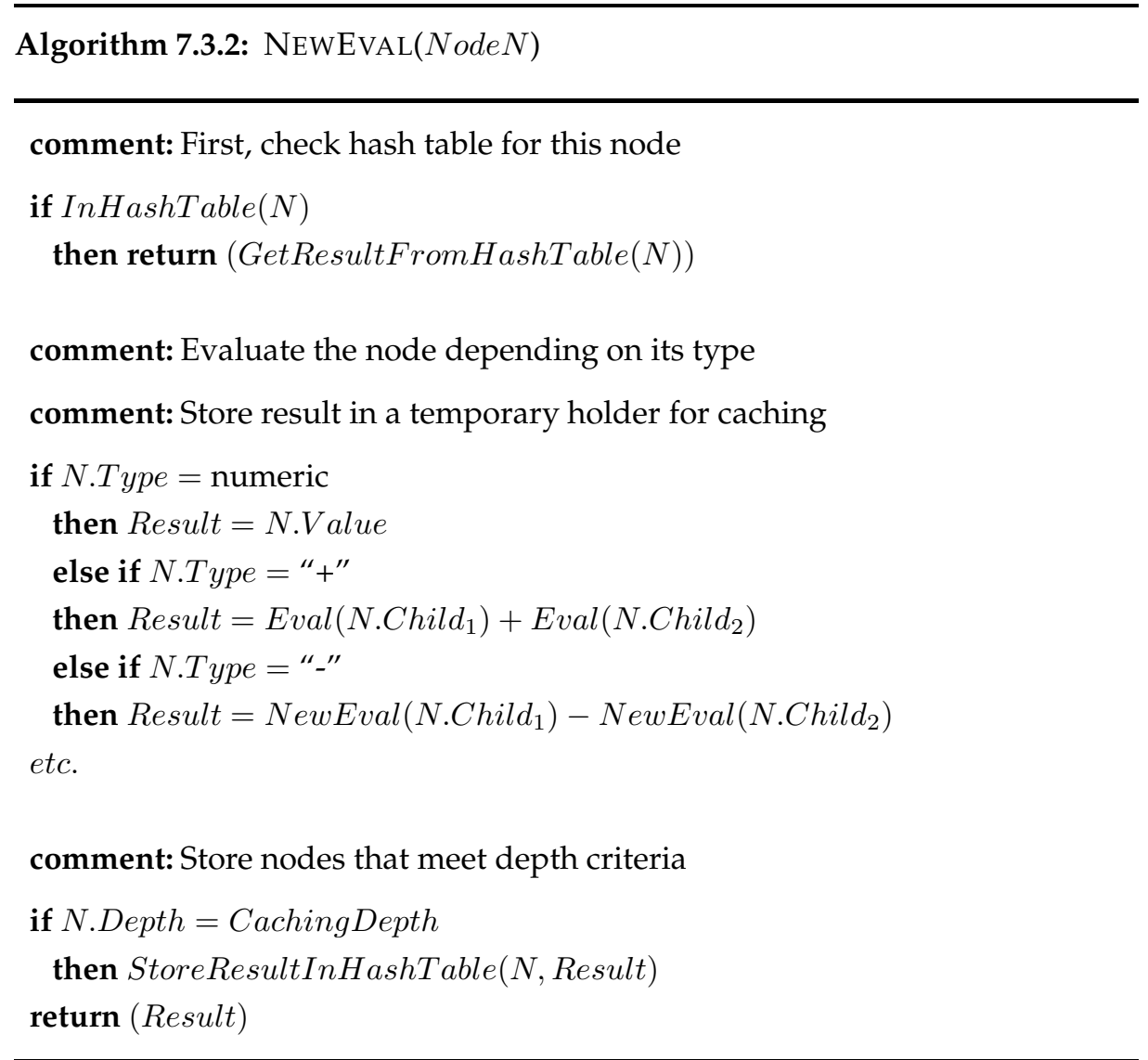

Figure 7.7: Pseudo-code for new Subtree Caching fitness evaluation method. 
what we define as a cache miss.

\section{Example}

To further illustrate the SCHEME approach, we present an example.

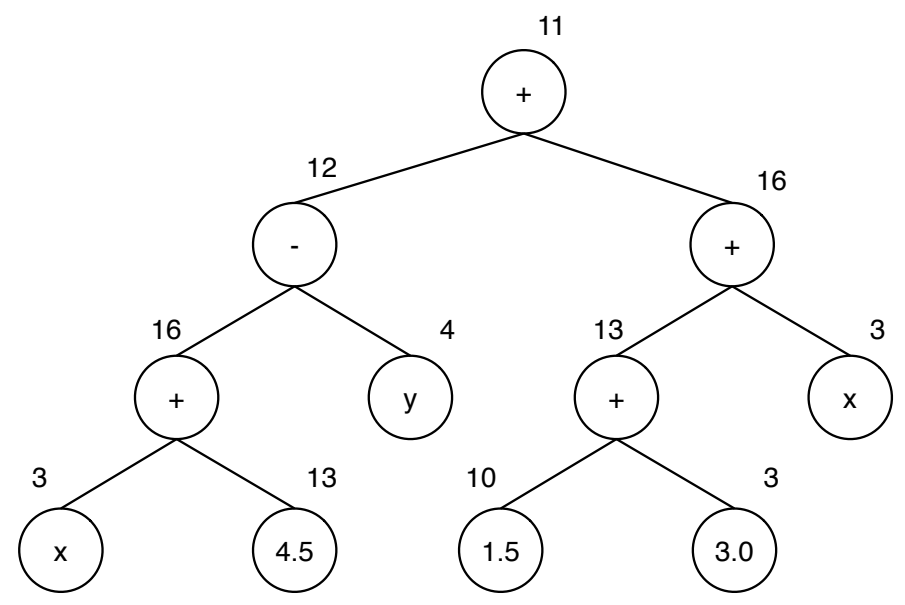

Figure 7.8: Example GP program used to demonstrate SCHEME, along with node hash values.

Figure 7.8 shows the GP program we will be using as an example here (along with pre-computed hash values for each program node shown above each node). We assume that the subtree cache is completely empty. The hash table size is 17 and the variables $x$ and $y$ have been randomly assigned hash values of 3 and 4 respectively. The caching depth we are using here is 1 (i.e. a single node with a number of child nodes). Our example fitness case will be $x=1.0$ and $y=2.0$.

Firstly, fitness evaluation traverses (following post-fix order) down from the root node to the bottom-left-most node in the tree $(x)$. As the cache is completely empty, none of the nodes can be located and no evaluation value fetching can be done. Since the $(x)$ node is a terminal node, caching is not applicable and the node is evaluated normally, resulting in a return value of $\langle 1.0\rangle$ (from our sole fitness case). Similarly, the next node visited (4.5) is also a terminal and returns a value of $<4.5>$. 
Moving up, the function node $(+)$ is also evaluated as normal, giving a return value of $\langle 5.5\rangle$. The depth of the subtree at this node is 1 , which meets our caching depth criteria. The evaluation value is stored in the cache, under entry 16 (which is the pre-calculated hash value for this subtree).

The $(y)$ and (-) nodes that make up the rest of the left-branch of the program are also evaluated as normal, giving the left branch a return value of $<3.5>$. These two nodes are of depth 0 and 2 respectively, and are not eligible for caching.

Now at this stage, fitness evaluation would usually traverse down to the bottom-left most node of the right branch (1.5) and then build up the return value for that whole branch. However, using SCHEME the cache is consulted at each of the nodes before any recursive call has taken placed. In this case, the subtree originating at the first $(+)$ node of the right branch has a hash value of 16 , which gives a cache hit. The evaluation value is retrieved from the cache, and so the $(+)$ returns the cached value of $<5.5>$. Finally, the root node is evaluated, giving the final program result of $<9.0>$. The fully evaluated program tree is displayed in figure 7.9.

In this simple example, a full fitness evaluation would usually require 11 node evaluations. However, by using SCHEME, fitness evaluation only requires 7 node evaluations. Also note that the subtree that was cached and the subtree that is using the cached value are not strictly identical, but are determined to be algebraically equivalent. As there are usually a large number of fitness cases used in GP tasks, we expect that this method can significantly reduce the node evaluation cost.

As the caching procedure is simply an extension of the standard fitness evaluation procedure, it can be easily added on to any implementation of the standard GP. 


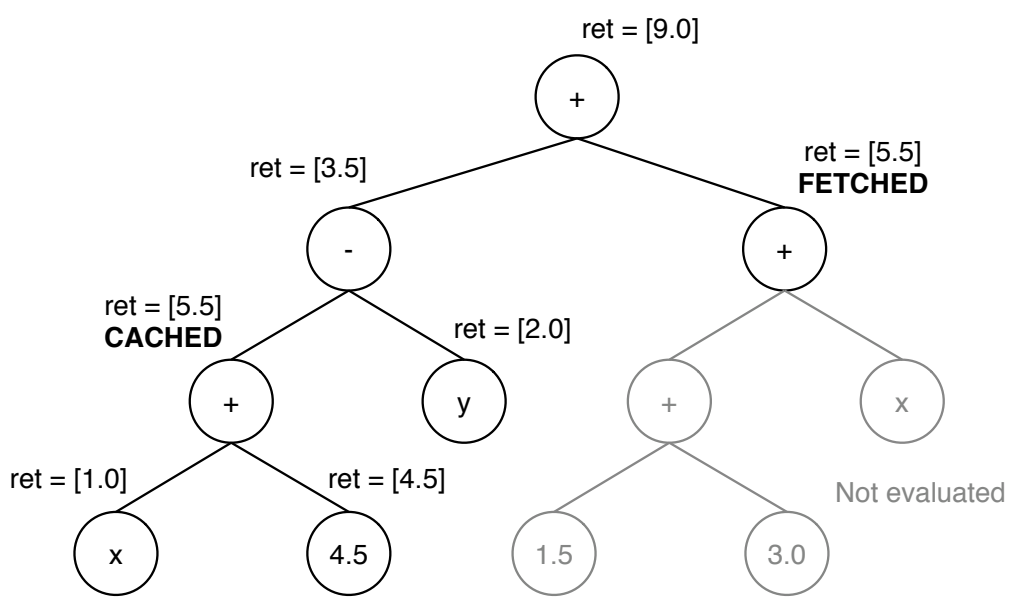

Figure 7.9: Example GP program, fully evaluated using SCHEME

\subsubsection{Experimental Setup}

For experimentation, six different datasets of varying difficulty were used, the first two being symbolic regression tasks and the remaining four being classification tasks (two image classification and two medical classification).

The six tasks chosen were: Regression 1 , Regression 2 , Shapes, Coins, Breast Cancer Wisconsin (BCW) and the SPECT dataset. For more information on these datasets, consult chapter 3 .

\section{Experiment Settings and Parameters}

Table 7.3 displays the parameters used for the GP systems, as well as the parameters used for the SCHEME method.

Note that a caching depth of 0 is not used in our experiments. Caching subtrees of depth 0 corresponds to caching singular nodes. Singular nodes already have the smallest cost of evaluation, and caching these is very likely to result in a worse performance, as we would be adding caching overhead for no gain. As we showed in section 7.3.1, caching subtrees above size 4 or 5 looks highly undesirable, hence in these experiments we restrict our cache depth to 5 and below. 
Table 7.3: GP and SCHEME parameters used for each dataset.

\begin{tabular}{c|c}
\hline Parameter & Value \\
\hline Pop. Size & 500 \\
Max. Gens. & 50 \\
Mutation & $30 \%$ \\
Crossover & $60 \%$ \\
Elitism & $10 \%$ \\
Max. Program Depth & 6 \\
\hline Hash Table Size & 499979 \\
Cache Depth & 1 to 5 (inclusive) \\
\hline
\end{tabular}

In order to ensure the validity of our results, each task was run for 50 GP runs for each of the system combinations tested (Standard GP, GP with SCHEME Depth 1, GP with SCHEME Depth 2, GP with SCHEME Depth 3, GP with SCHEME Depth 4 and GP with SCHEME Depth 5), making up 1800 runs in total (50 runs $\times 6$ tasks $\times 6$ systems). Each GP run was given a different random seed in order to provide different initial conditions, and these seeds were kept consistent between systems so that each system was given the same 50 random seeds for their set of GP runs.

To reduce any possible variability in recorded CPU times, all of the experiments were performed on a single Dell Optiplex GX745 machine with 2GB of memory.

\subsubsection{Results}

In this section we discuss the results that we obtained from the experiments performed using the six tasks.

\section{SCHEME vs. Standard GP}

Table 7.4 displays a comparison of the best results between the Standard GP and the GP with SCHEME methods. The results display the CPU time 
Table 7.4: Comparison table of Standard GP versus SCHEME for all tasks.

\begin{tabular}{|c|c|c|c|c|}
\hline \multirow[t]{2}{*}{ Task (No. Cases) } & \multicolumn{2}{|c|}{ Regression $_{1}(400)$} & \multicolumn{2}{|c|}{ Regression $_{2}(200)$} \\
\hline & Standard & Caching & Standard & Caching \\
\hline Time & $2.224 \pm 0.456$ & $1.673 \pm 0.198$ & $1.810 \pm 0.168$ & $1.409 \pm$ \\
\hline Time Reduction & \multicolumn{2}{|c|}{$24.78 \%$} & \multicolumn{2}{|c|}{$22.15 \%$} \\
\hline Node Eval's & $758309 \pm 152637$ & $202989 \pm 25045.9$ & $890984 \pm 94795.1$ & $198126 \pm$ \\
\hline Eval Reduction & \multicolumn{2}{|c|}{$73.23 \%$} & \multicolumn{2}{|c|}{$77.76 \%$} \\
\hline Cache & $\mathrm{N} / \mathrm{A}$ & $65124.9 \pm 10631.9$ & $\mathrm{~N} / \mathrm{A}$ & $75499.1 \pm 7307.62$ \\
\hline Cache Misses & $\mathrm{N} / \mathrm{A}$ & $12764.7 \pm 1265$ & $\mathrm{~N} / \mathrm{A}$ & $12406.4 \pm 817.965$ \\
\hline \multirow[t]{2}{*}{ Task (No. Cases) } & \multicolumn{2}{|c|}{ Shapes (1800) } & \multicolumn{2}{|c|}{ Coins (385) } \\
\hline & Standard & Caching & Standard & Caching \\
\hline Tim & $3.051 \pm 0.375$ & $2.311 \pm 0.163$ & $1.469 \pm 0.266$ & $1.359 \pm 0.176$ \\
\hline Time Reduction & \multicolumn{2}{|c|}{$24.25 \%$} & \multicolumn{2}{|c|}{$7.49 \%$} \\
\hline Node Eval's & $782078 \pm 108980$ & $271886 \pm 24392.3$ & $736253 \pm 131099$ & $296646 \pm 30284.9$ \\
\hline Eval Reduction & \multicolumn{2}{|c|}{$65.24 \%$} & \multicolumn{2}{|c|}{$59.71 \%$} \\
\hline Cache Hits & N/A & $82490.4 \pm 16286.6$ & N/A & $49685.6 \pm 11625.9$ \\
\hline Cache Misses & $\mathrm{N} / \mathrm{A}$ & $15651.8 \pm 641.73$ & $\mathrm{~N} / \mathrm{A}$ & $16037.9 \pm 999.507$ \\
\hline \multirow[t]{2}{*}{ Task (No. Cases) } & \multicolumn{2}{|c|}{$\overline{~ B C W ~(699) ~}$} & \multicolumn{2}{|c|}{ SPECT (267) } \\
\hline & Standard & Caching & Standard & Caching \\
\hline Time & $1.955 \pm 0.244$ & $1.664 \pm 0.153$ & $1.514 \pm 0.131$ & $1.375 \pm 0.133$ \\
\hline Time Reduction & \multicolumn{2}{|c|}{$14.88 \%$} & \multicolumn{2}{|c|}{$9.18 \%$} \\
\hline Node Eval's & $803272 \pm 103514$ & $312373 \pm 24821.9$ & $829208 \pm 85301.9$ & $289273 \pm 18083.5$ \\
\hline Eval Reduction & \multicolumn{2}{|c|}{$61.11 \%$} & \multicolumn{2}{|c|}{$65.11 \%$} \\
\hline Cache Hits & $\mathrm{N} / \mathrm{A}$ & $50173 \pm 9936.2$ & $\mathrm{~N} / \mathrm{A}$ & $83264.4 \pm 16063.9$ \\
\hline Cache Misses & $\mathrm{N} / \mathrm{A}$ & $16277.6 \pm 623.441$ & $\mathrm{~N} / \mathrm{A}$ & $16885.2 \pm 940.366$ \\
\hline
\end{tabular}


taken for the GP system to run (Time) and the number of node evaluations performed for the entire GP run. Additionally, for the GP system using SCHEME, the numbers of cache hits and misses are also shown. The reduction in Time and Evaluations obtained by using SCHEME is given for each task as a percentage.

One of the major goals of this chapter was to reduce the cost of evaluating fitness cases. The results summarised in table 7.4 clearly show that for all six tasks, using SCHEME resulted in a large reduction in the number of nodes being evaluated in a GP run. For all of the tasks, the reduction in node evaluations exceeded 59\%. In particular, for the two regression problems, the number of node evaluations was reduced by over $70 \%$. This may be because of the repetitive nature of the problems used $((8 x+8)$ is the same as $8 \cdot(x+1)$ and $(x+2)^{3}$ is simply $\left.(x+2)(x+2)(x+2)\right)$, which may result in more cache hits. Indeed, for those tasks that performed best at caching depth 3 , the two regression problems had the highest cache hit rate.

The results in table 7.4 also detail the CPU times required for a GP run. They show that using SCHEME resulted in reduction of between $7 \%$ and $25 \%$ in the time required to train the GP programs. The amount of reduction is significantly lower than the amount that SCHEME lowered the number of node evaluations, which suggests that there is a certain amount of overhead that SCHEME introduces that must be overcome in order to gain improvements in GP training times. The impact that caching has on the GP system can be affected by many parameters such as population size, number of generations, number of fitness cases, computation costs of functions.

Hashing merely provides an estimation of algebraic equivalence, which of course can lead to hashing collisions. This may lead to some indirect alteration of the GP populations. It is important that using the caching method does not deteriorate the performance or effectiveness of the GP system. If a method were to worsen the effectiveness of the GP system, then 
Table 7.5: Average Training and Testing Fitnesses of Solution Programs for all six tasks. Fitness for the Regression problems is the measured MSE, while for the other tasks it is the classification accuracy.

\begin{tabular}{c|cc|cc}
\hline & \multicolumn{2}{|c}{ Training Fitness } & \multicolumn{2}{c}{ Testing Fitness } \\
\hline Dataset & Standard GP & SCHEME & Standard GP & SCHEME \\
\hline Regression $_{1}$ & $0.0838 \pm 0.193$ & $0.0783 \pm 0.208$ & N/A & N/A \\
Regression $_{2}$ & $1718.06 \pm 2090.91$ & $1836.76 \pm 2108.5$ & N/A & N/A \\
Shapes & $0.887 \pm 0.163$ & $0.913 \pm 0.106$ & $0.883 \pm 0.166$ & $0.908 \pm 0.109$ \\
Coins & $0.761 \pm 0.145$ & $0.764 \pm 0.150$ & $0.761 \pm 0.150$ & $0.751 \pm 0.184$ \\
BCW & $0.856 \pm 0.108$ & $0.866 \pm 0.128$ & $0.912 \pm 0.105$ & $0.914 \pm 0.131$ \\
SPECT & $0.884 \pm 0.125$ & $0.897 \pm 0.149$ & $0.896 \pm 0.137$ & $0.915 \pm 0.151$ \\
\hline
\end{tabular}

this would of course make the caching method less desirable to use. The experimental results gained on all six tasks showed very small differences in the training and testing fitnesses obtained (the training and testing fitnesses for the best results in both the standard GP and SCHEME methods are displayed in table 7.5). Using a statistical Z-Test to pairwise compare the caching systems with the standard GP, we found that there was no significant difference (to a 95\% confidence interval) in either the training fitnesses or testing fitnesses (in applicable tasks) yielded by these systems. This suggests that using SCHEME has no significant impact on the effectiveness of the GP system.

Overall, the results show that by using SCHEME we can significantly reduce both the number of node evaluations as well as the GP training time, improving the efficiency of GP. More importantly, this improvement does not come at the cost of GP effectiveness, as a statistical Z-test shows no significant difference in solution accuracy on both training and test data. 


\subsection{Discussions of Caching Depth}

Firstly, using SCHEME at each of the caching depths tested (1 to 5) led in almost all cases to a decrease in both Node Evaluations and Time compared to the Standard GP (only in the SPECT task did a caching depth of 5 have a higher CPU Time than Standard GP). However, the caching depth that performed best was always either 2 or 3, depending on the task and the measurement (Node Evaluations or CPU Time). The number of node evaluations and CPU times for all six tasks are illustrated in figure 7.10.

When considering node evaluations, the four classification tasks had the lowest number of node evaluations when using caching depth 2 (Shapes, Coins, Breast Cancer Wisconsin and SPECT), while the two symbolic regression problems performed best with caching depth 3 . However, when considering CPU times, four of the tasks had the lowest times when using depth 3 (Regression $_{1}$, Regression 2 , Coins and Breast Cancer Wisconsin), and the remaining two with caching depth 2 (Shapes and SPECT). It is notable that the differences between the times for the two depths are small. However the results indicate that the lowest number of node evaluations may not directly correspond with the lowest CPU time.

With regards to the other caching depths, a caching depth of 1 appears to perform better than a caching depth of 4 in tasks where the number of node evaluations was lowest at depth 2 . While a caching depth of 4 outperformed a caching depth of 1 in tasks where the number of node evaluations was lowest at depth 3 . Whichever of these is closer to the optimal depth outperforms the other. A caching depth of 5 always performed the poorest out of the 5 tested depths.

The reason that a caching depth of 1 may not be optimal is that using this depth caches the smallest possible trees which are not singular. This results in a lot of caching activity, and while there may be a lot of cache hits when using this depth, these hits do not save many node evaluations. In this case, the overhead from performing caching may cancel a large 

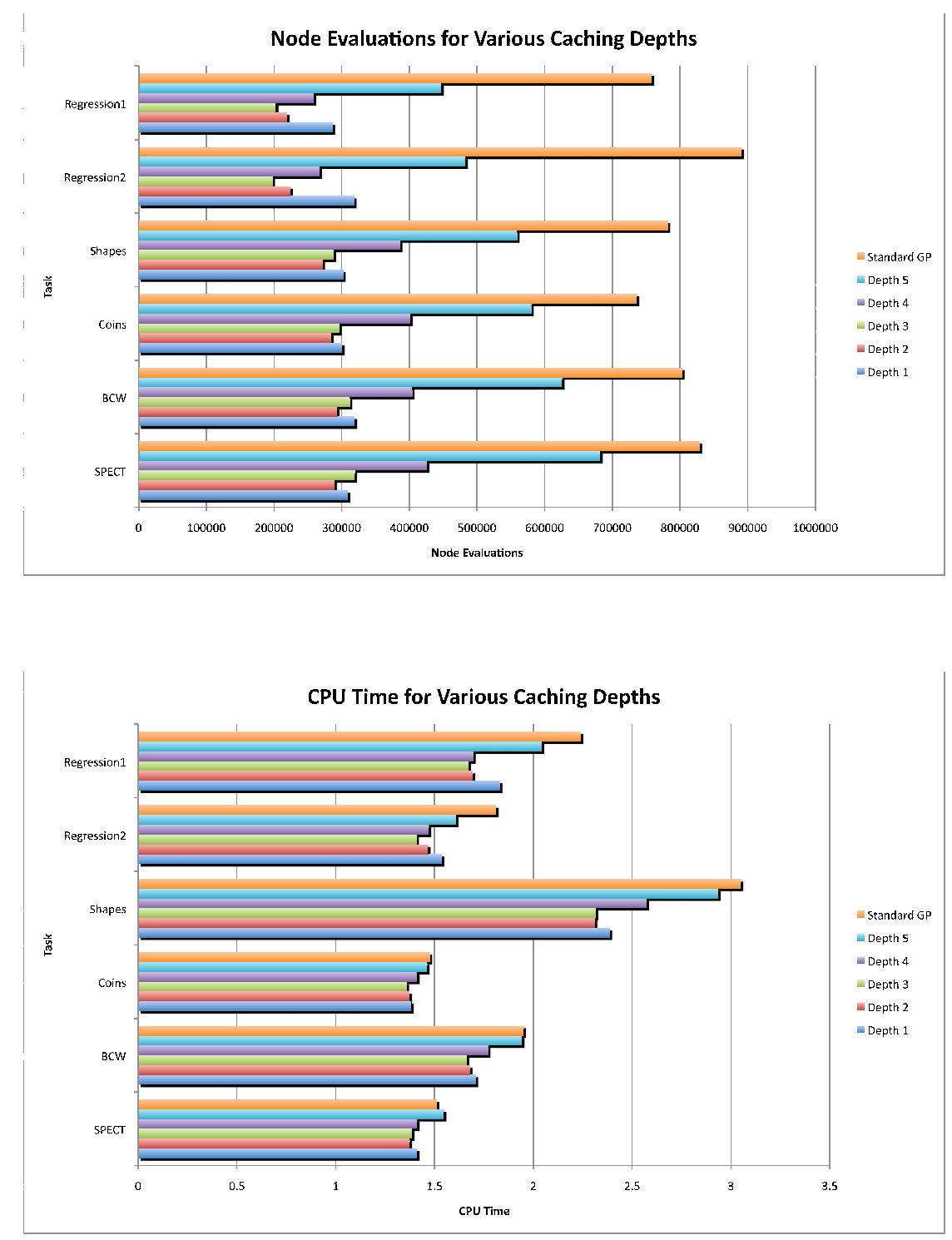

Figure 7.10: No. of Node Evaluations and CPU Time when caching depth is varied.

amount of the benefit of the subtree caching. 
Conversely, using a caching depth of 4 or 5 means that fairly large subtrees are being cached. While this can mean saving a lot of node evaluations whenever the cache is used, there are exponentially more different subtrees of larger size. This means that the number of cache hits will be somewhat reduced, resulting in worse performance than using a smaller cache depth.

As all of the tasks performed best when a caching depth of 2 or 3 were used, we then tried to cache both subtrees of depth 2 and subtrees of depth 3. Performing the experimental tasks when caching both depths 2 and 3 did not yield better results than simply caching either depth 2 or depth 3 .

Overall, the results showed that when using a caching depth that is too small, not enough node evaluations are being saved per cache hit. Conversely, when using a caching depth that is too large, there are too few cache hits to be effective. The results gathered on the six GP tasks empirically show that a caching depth of 2 or 3 may be a good starting point when selecting a caching depth for other GP tasks.

\subsection{Discussions on Increasing the Number of Fit- ness Cases}

Each of the datasets used have varying numbers of available data instances. This means that for each node evaluated, GP would have to evaluate that node for a different number of instances, depending on which dataset is used. This obviously can have an effect on the amount of computation effort saved when using SCHEME. This subsection will look at how the number of fitness cases in a dataset affects the efficiency when using SCHEME.

For experimentation we used an expanded version of the Shapes dataset. This dataset is the same as the original Shapes dataset (with 1800 instances), but has had additional instances generated (using the original programs 
used to randomly generate this artificial dataset) making a total of 30,000 data instances. We then ran GP with SCHEME on subsets of this dataset, varying in size $(1800 ; 3000 ; 4800 ; 7200 ; 9000 ; 15,000 ; 30,000)$. By varying the number of fitness cases, we get a measure for how the reductions gained by SCHEME in computational cost scale as the number of fitness cases is increased.

\section{Results}

Figure 7.11 shows the average CPU time and number of evaluations GP obtained on each of the dataset subsets. Figure 7.12 shows the number of cache hits and number of cache misses that occurred when using GP on each of the dataset subsets.

As shown by their graphs, the Number of Evaluations, Number of Caching Hits and Number of Caching Misses remain relatively consistent when increasing the number of data instances. However, the graph for CPU Time shows an increasing amount of time being saved when using SCHEME, with the gap between the time for the standard GP and the time for the GP using SCHEME continually widening. This is because as the number of fitness instances increases, the time required for evaluating each GP program also increases. Multiplied across all of the programs in all of the generations during the GP evolution, fitness evaluation as a process takes up a larger and larger proportion of the GP evolution effort. As SCHEME reduces the number of program nodes that need to be evaluated and each node would need to be ordinarily evaluated for a large number of fitness cases, by increasing the number of fitness cases we are increasing the amount of effort that SCHEME saves. This leads to the widening gap between the time obtained using standard GP and the time obtained when using the SCHEME method.

This CPU time graph shows that the CPU time does not increase linearly as the number of fitness cases increases, and may grow at an exponential or geometric rate. This implies that SCHEME is highly suitable 


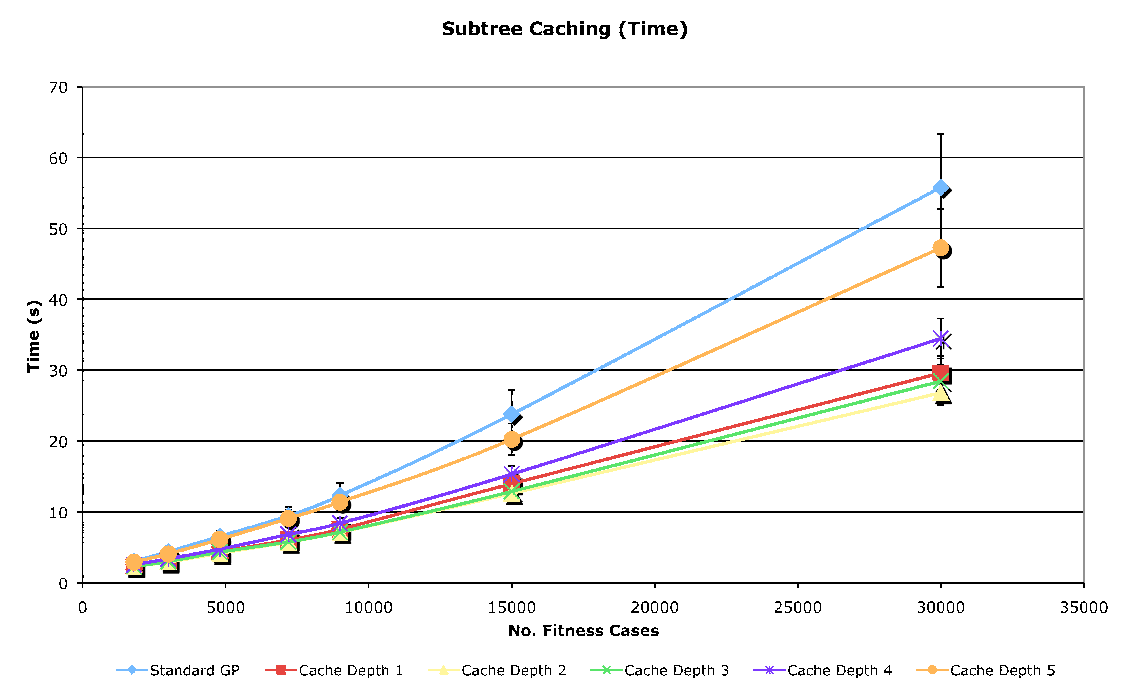

CPU Time

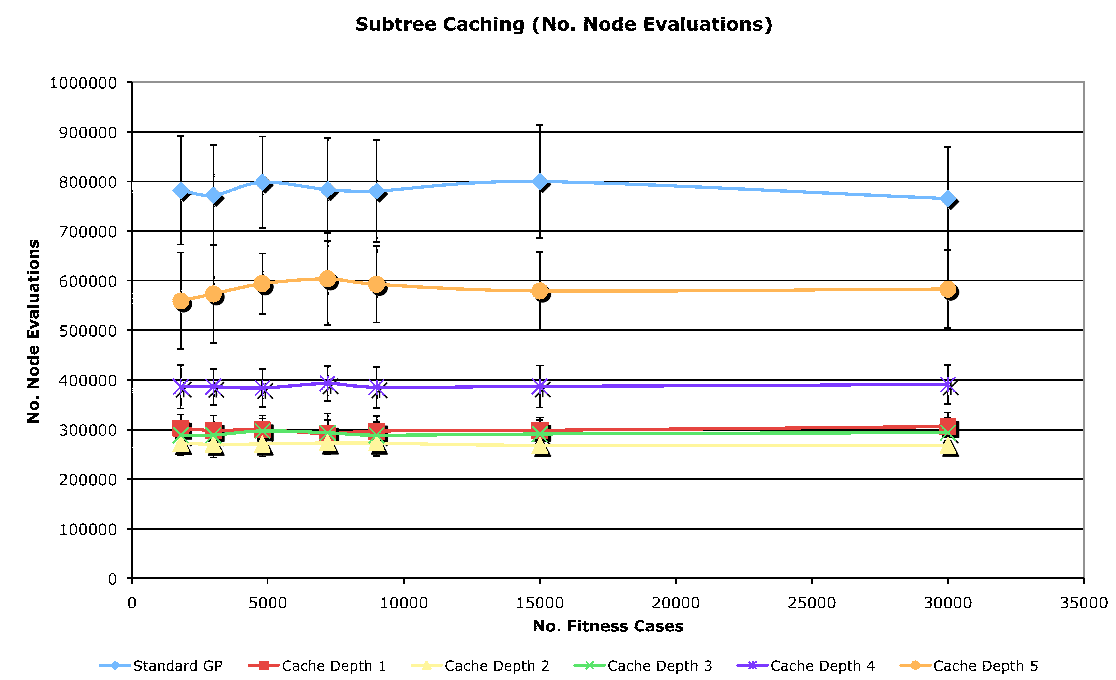

Node Evaluations

Figure 7.11: CPU Time and Node Evaluations for using SCHEME on Shapes datasets on increasing size 


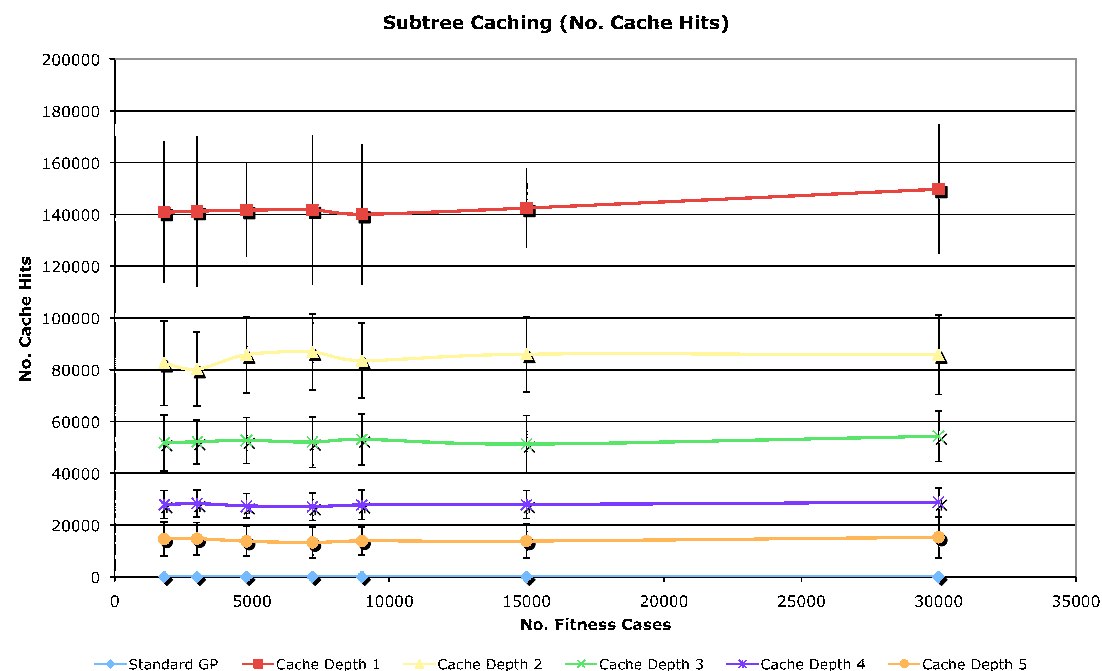

Cache Hits

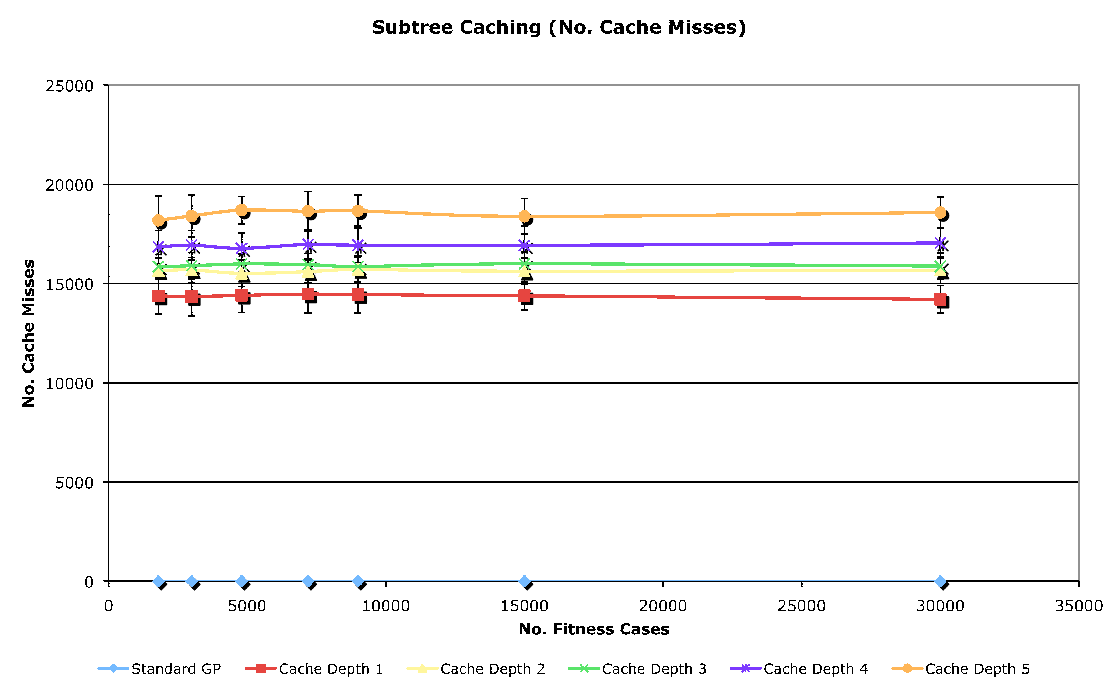

Cache Misses

Figure 7.12: Cache Hits and Cache Misses for using SCHEME on Shapes datasets on increasing size 


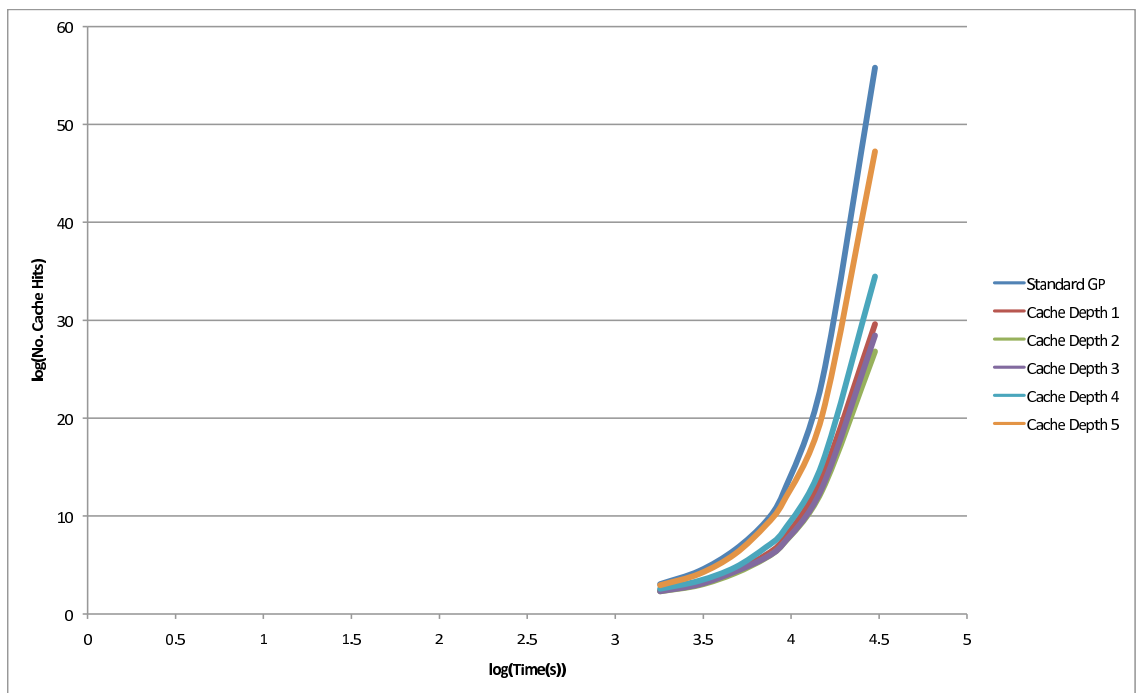

(a)

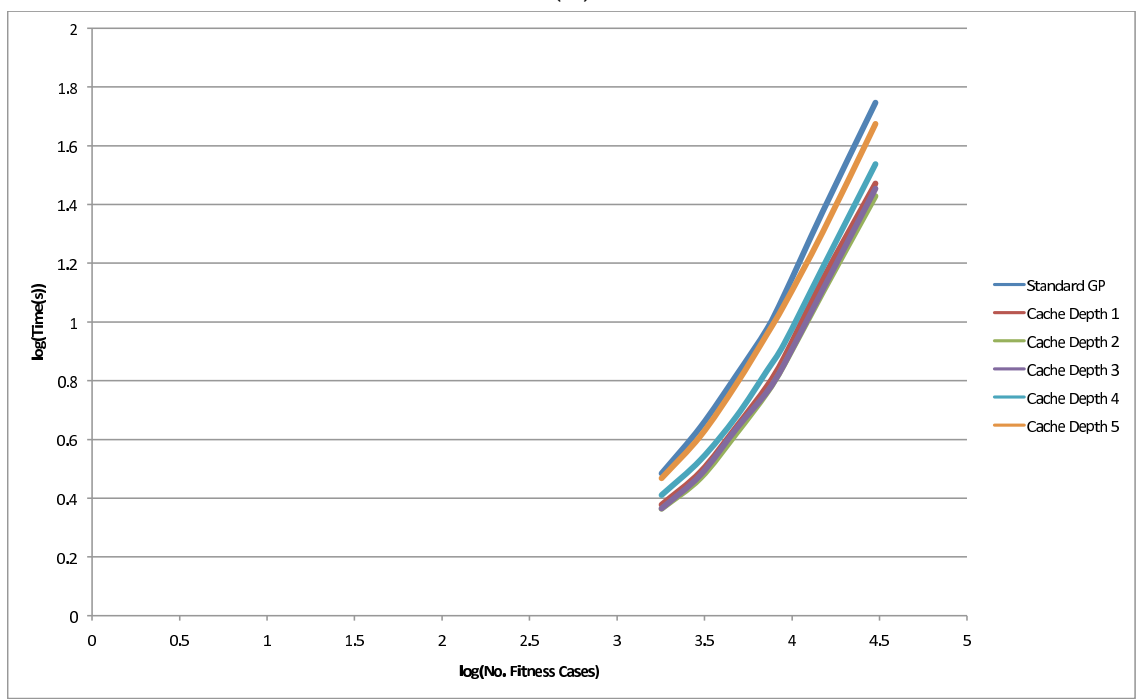

(b)

Figure 7.13: CPU Time for using SCHEME on Shapes datasets on increasing size: (a) $\log$ (No. Fitness Cases) vs. Time(s), (b) $\log$ (No. Fitness Cases) vs. $\log$ (Time(s)) 
for tasks which contain a very large number of fitness cases. Figure 7.13 shows more CPU time graphs, the first plotting $\log$ (No. Fitness Cases) vs. Time(s) and the second plotting $\log$ (No. Fitness Cases) vs $\log$ (Time(s)). The first graph does not show a linear relationship between $\log$ (No. Fitness Cases) and Time(s), which tells us that the relationship between CPU time and the number of fitness cases is not exponential. However, the second plot does suggest a linear relationship between $\log$ (No. Fitness Cases) and $\log ($ Time(s)), suggesting a power law relationship between CPU time and the number of fitness cases in the training set.

\subsection{Selecting Caching Depth Dynamically}

One of the major conclusions that were found from experiments performed using SCHEME was that the optimal caching depth can vary for different GP tasks. Also, in standard SCHEME the caching depth is kept constant for the entirety of the GP evolution. In this section, we explore methods of dynamically selecting the caching depth during the GP evolution.

\subsection{1 dSCHEME: Caching Dynamically Chosen Subtrees}

This is more complex subtree selection mechanism that is similar to that used in "Alternatives in Subtree Caching". It is based upon the notion that if both of child nodes are being used often in the cache, then we should add this parent node as it is likely to be used often as well, and incrementally save more node evaluations.

Every time a subtree is fetched from the subtree cache, a count of how many times it is used is incremented. This keeps track of how often a particular subtree is used since it was cached (our early experiments suggest that only around $4 \%$ of subtrees are used more than 10 times and $40 \%$ are used at least once). This way it is easy to find which subtrees are being used often. 
Table 7.6: Comparison table of dSCHEME versus SCHEME for all tasks.

\begin{tabular}{c|cc|cc}
\hline \multirow{2}{*}{ Task (No. Cases) } & \multicolumn{2}{|c|}{ Regression 1400$)$} & \multicolumn{2}{c}{ Regression 2 (200) } \\
& dSCHEME & Caching & dSCHEME & Caching \\
\hline Time (s) & $1.686 \pm 0.209$ & $1.673 \pm 0.198$ & $1.488 \pm 0.144$ & $1.409 \pm 0.105$ \\
\hline Node Eval's & $237614 \pm 38309.7$ & $202989 \pm 25045.9$ & $242140 \pm 42168.7$ & $198126 \pm 16420.5$ \\
\hline Cache Hits & $101246 \pm 16813.3$ & $65124.9 \pm 10631.9$ & $115517 \pm 15799$ & $75499.1 \pm 7307.62$ \\
Cache Misses & $12099 \pm 1306.26$ & $12764.7 \pm 1265$ & $11735.4 \pm 562.268$ & $12406.4 \pm 817.965$ \\
\hline \hline Task (No. Cases) & \multicolumn{2}{|c|}{ Shapes (1800) } & \multicolumn{2}{c}{ Coins (385) } \\
& dSCHEME & Caching & dSCHEME & Caching \\
\hline Time (s) & $2.381 \pm 0.193$ & $2.311 \pm 0.163$ & $1.41 \pm 0.216$ & $1.359 \pm 0.176$ \\
\hline Node Eval's & $261922 \pm 33950.2$ & $271886 \pm 24392.3$ & $265846 \pm 38834.2$ & $296646 \pm 30284.9$ \\
\hline Cache Hits & $109722 \pm 17687.2$ & $82490.4 \pm 16286.6$ & $110090 \pm 19552.5$ & $49685.6 \pm 11625.9$ \\
Cache Misses & $14271.7 \pm 895.072$ & $15651.8 \pm 641.73$ & $14088.1 \pm 1223.52$ & $16037.9 \pm 999.507$ \\
\hline \hline Task (No. Cases) & \multicolumn{2}{|c|}{$B C W$} & $699)$ & \multicolumn{2}{c}{ SPECT (267) } \\
& dSCHEME & Caching & dSCHEME & Caching \\
\hline Time (s) & $1.742 \pm 0.184$ & $1.664 \pm 0.153$ & $1.426 \pm 0.134$ & $1.375 \pm 0.133$ \\
\hline Node Eval's & $281183 \pm 33464.6$ & $312373 \pm 24821.9$ & $267624 \pm 29885.2$ & $289273 \pm 18083.5$ \\
\hline Cache Hits & $119563 \pm 16134.6$ & $50173 \pm 9936.2$ & $117036 \pm 13769.6$ & $83264.4 \pm 16063.9$ \\
Cache Misses & $14264.9 \pm 795.157$ & $16277.6 \pm 623.441$ & $15849.6 \pm 1120.67$ & $16885.2 \pm 940.366$ \\
\hline
\end{tabular}

At first, only subtrees of depth 1 are cached, as these are the smallest possible non-singular subtrees. For trees which are of bigger depth (2+), the child nodes are looked up in the cache. If both of these child nodes are in the cache, then their subtree cache use counts are checked. If both of these counts are sufficiently high (controlled by a specified parameter), then the bigger subtree is also cached. The aim is to have in the cache the biggest possibly sized subtrees that are used very often. If a bigger subtree is not used very often then its parent will not be added to the cache and the growth stops there.

Results from all six tasks as well as the results from SCHEME using the "best" static caching-depths are given in table 7.6. In the four classification tasks (Shapes, Coins, BCW and SPECT) using dSCHEME reduces the number of node evaluations when compared to the standard GP. How- 
ever, in the regression tasks (Regression ${ }_{1}$ and Regression ${ }_{2}$ ) there is an increase in the number of node evaluations. Even with a decrease in node evaluations in four of the six tasks, there was an increase in CPU time for all six tasks. This is likely because of the increased overhead needed to track subtree counts and check that child nodes are (a) already in the subtree cache and (b) have a sufficiently high use count. It appears that a selection method will need very little overhead to be effective.

\subsection{2 iSCHEME: Caching using Incremental Depth}

We have seen in our results from dSCHEME that the dynamic cachingdepth selection method will need to be very fast in order to not incur too much overhead that will offset the savings that reducing the number of node evaluations gives. Here, we will look at a much simpler approach to selecting caching depth.

Initial GP populations are usually generated entirely at random. This means that any shared subtrees between any two programs is entirely up to chance, which as we showed in subsection 7.3.1 becomes increasingly unlikely as the subtree size considered increases. This suggests that as we begin our GP evolution, we should only be caching very small subtrees as they are the most likely to have repetitions in the population. As the GP evolution progresses, the GP population tends to converge and it is likely that larger and larger subtrees will repeat themselves in the program population. This suggests that we should increase the caching size as evolution progresses to benefit most from subtree caching.

Based on this idea, we developed iSCHEME ("incremental SCHEME"). This method begins with a caching depth of 1 (the smallest non-singular subtree possible) and increments the depth at regular intervals in the evolution run until the last interval of GP generations are caching whole programs. Our implementation calculates the cache depth, given the current generation number as follows: 
Table 7.7: Comparison table of iSCHEME versus SCHEME for all tasks.

\begin{tabular}{c|cc|cc}
\hline \multirow{2}{*}{ Task (No. Cases) } & \multicolumn{2}{|c|}{ Regression $_{1}(400)$} & \multicolumn{2}{c}{ Regression $_{2}(200)$} \\
& iSCHEME & Caching & iSCHEME & Caching \\
\hline Time (s) & $1.650 \pm 0.192$ & $1.673 \pm 0.198$ & $1.416 \pm 0.127$ & $1.409 \pm 0.105$ \\
\hline Node Eval's & $190007 \pm 30627.9$ & $202989 \pm 25045.9$ & $191925 \pm 15632.9$ & $198126 \pm 16420.5$ \\
\hline Cache Hits & $82127.5 \pm 10154.9$ & $65124.9 \pm 10631.9$ & $91668.6 \pm 8173.82$ & $75499.1 \pm 7307.62$ \\
Cache Misses & $15336.3 \pm 2084.42$ & $12764.7 \pm 1265$ & $15179.4 \pm 862.77$ & $12406.4 \pm 817.965$ \\
\hline \hline Task (No. Cases) & \multicolumn{2}{|c|}{ Shapes (1800) } & \multicolumn{2}{c}{ Coins (385) } \\
& iSCHEME & Caching & iSCHEME & Caching \\
\hline Time (s) & $2.278 \pm 0.172$ & $2.311 \pm 0.163$ & $1.383 \pm 0.141$ & $1.359 \pm 0.176$ \\
\hline Node Eval's & $226517 \pm 17506.7$ & $271886 \pm 24392.3$ & $232415 \pm 14650.1$ & $296646 \pm 30284.9$ \\
\hline Cache Hits & $83536.7 \pm 9138.05$ & $82490.4 \pm 16286.6$ & $85042.9 \pm 9984.28$ & $49685.6 \pm 11625.9$ \\
Cache Misses & $18617.2 \pm 584.672$ & $15651.8 \pm 641.73$ & $19299.2 \pm 496.094$ & $16037.9 \pm 999.507$ \\
\hline \hline Task (No. Cases) & \multicolumn{2}{|c|}{$B C W$} & $699)$ & \multicolumn{2}{c}{ SPECT (267) } \\
& iSCHEME & Caching & iSCHEME & Caching \\
\hline Time (s) & $1.672 \pm 0.161$ & $1.664 \pm 0.153$ & $1.401 \pm 0.139$ & $1.375 \pm 0.133$ \\
\hline Node Eval's & $238269 \pm 14769.4$ & $312373 \pm 24821.9$ & $246536 \pm 24151.5$ & $289273 \pm 18083.5$ \\
\hline Cache Hits & $89563.6 \pm 10465.4$ & $50173 \pm 9936.2$ & $88898.1 \pm 9527.79$ & $83264.4 \pm 16063.9$ \\
Cache Misses & $19706.6 \pm 446.691$ & $16277.6 \pm 623.441$ & $20902.1 \pm 655.039$ & $16885.2 \pm 940.366$ \\
\hline
\end{tabular}

$$
\text { cache depth }=\frac{\text { current gen }}{\left(\frac{\text { max. gens }}{\text { max. tree depth }-1}\right)}
$$

Note: It is important to note that the maximum tree depth parameter regards a depth of 1 to be a singular node, while in SCHEME we define a depth of 1 as the smallest non-singular subtree (i.e. two levels). This is the reason that our equation uses (max. tree depth - 1).

For example, consider a GP setup where the maximum number of generations is 50 , and the maximum tree depth allowed is 6 . This means that the cache depth is calculated by $\frac{\text { current gen }}{50 \div 5}=\frac{\text { current gen }}{10}$. This essentially means that every 10 generations the caching depth is incremented. At the last generation $\left(50^{\text {th }}\right)$ the caching depth becomes 6 , meaning that no caching takes place in the final generation. 
Results for iSCHEME are given in table 7.7. These clearly show that in all of the tasks that the number of node evaluations is further reduced when compared to the "best" depths found for each task when using standard SCHEME. However, this reduction in node evaluations does not directly translate into proportional savings in time, with iSCHEME performing slightly poorer than the "best" depths in all tasks except the Shapes dataset. This suggests that there may be some level of overhead from calculating cache depths as evolution progresses. As this method is very simple, finding an effective method of selecting caching depth may be difficult.

In both our results from dSCHEME and iSCHEME, we see that we can achieve lower node evaluations using both of these methods. However, the overhead that these selection methods add appears to overpower the reduction in node evaluations they achieve and the CPU times generally increase over the "best" caching-depth using regular SCHEME. It appears that finding a good dynamic selection scheme is difficult and would require a combination of low overhead and very high reduction of node evaluations in order to reduce CPU times.

\subsection{Chapter Summary}

In this chapter, we have aimed to reduce the code of performing fitness evaluation in GP. Our first method we developed (in line with our first goal) was elitism avoidance, where programs in a new generation that were created through elitism reproduction were not evaluated for fitness, and their fitness simply copied from the old generation. Though this method was simple, our results on six GP tasks showed that using elitism avoidance could significantly reduce the CPU time required by GP to evolve a solution program. How much CPU time could be saved was found to be task dependent, but when using a "typical" elitism rate of $10 \%$, we found that CPU time was reduced between $3.5 \%$ and $7 \%$. 
We then described Subtree Caching using a Hashing for Equivalence MEthod (SCHEME), our method for reducing the cost of fitness evaluations which utilises Subtree Caching and Algebraic Equivalence Hashing. The SCHEME method requires setting a new parameter: caching depth, which determines the size of subtrees to store in the cache. A GP system using SCHEME is compared to the standard GP system using six different tasks of varying difficulty. The results showed that by using SCHEME, there was a large reduction in the number of node evaluations that were performed in evaluating the GP programs during the GP runs. The results also showed a significant reduction in the CPU time required to perform each GP run, although this reduction varied with each task. Statistical significance testing showed that SCHEME did not significantly affect the training fitnesses and testing accuracies obtained on the six tasks, showing that this is a viable method of reducing the costs of evaluation without deteriorating GP effectiveness.

While with all caching depths, using SCHEME resulted in improved efficiency, it was found that caching subtrees of depth 2 or 3 was the most effective. Four out of the six tasks had the lowest CPU times with a caching depth of 3 , and four out of the six tasks had the lowest number of node evaluations when using a caching depth of 2 . This suggests that obtaining the lowest number of node evaluations may not equate to the lowest CPU time (although the times for these two depths were close) and this phenomenon needs further investigation. A caching depth of 2 or 3 may be a good starting point when using this caching approach on other GP tasks.

The optimal value for caching depth is task specific. We therefore aimed to develop a method of dynamically selecting the caching depth for use with the SCHEME method. This was performed in two ways. The first was to begin with the smallest caching depth and increment the depth at regular intervals during the GP evolution (iSCHEME). The second was to track the cache activity for subtrees and only cache subtrees that contained frequently used subtress (dSCHEME). Both of these methods were used on 
the same six GP tasks as the original SCHEME and the results of all three compared. It was found that while using these dynamic methods reduces the number of node evaluations and improves cache efficiency over the standard GP, the CPU time required for GP runs using these methods was generally worse than when using the "optimal" cache depth with the original SCHEME. This is most likely caused by the extra overhead introduced by the caching depth selection algorithms. In future work we will look at other methods of dynamically selecting the caching depth. 
164 CHAPTER 7. REDUCING COSTS OF FITNESS EVALUATIONS 


\section{Chapter 8}

\section{Conclusions}

In this chapter, we present the contributions and conclusions of the body of work in this thesis, as related to the research questions posed in section 1.3. We then outline some possible future research directions.

\subsection{Conclusions}

In this thesis, our overall goal was to increase the efficiency of GP, thereby improving its utilisation of available system resources. To achieve this, we have focused on two current issues in GP: Program Bloat, which is the tendency of GP programs to grow rapidly with often useless/redundant code; and Fitness Evaluation, which is the most costly process in GP, especially when the number of available fitness cases are numerous. The overall goal has been achieved through our research in these two areas, and has led to several new methods. In the rest of this section, we summarise the major conclusions that directly correspond to our research questions. Then we describe our major findings that were not directly related to our research questions, but derived from our results. 


\subsubsection{Direct Conclusions}

- We developed a new method of removing redundant GP program code (Algebraic Simplification) using algebraic rules and hashing. We showed that it could significantly reduce the size of programs within the GP populations and the CPU time that GP uses to evolve solutions. We found that using simplification does not reduce the effectiveness of the GP system in finding solutions.

Our Algebraic Simplification method used simple algebraic rules and an equivalence hashing technique (which determined the equivalence of GP program subtrees) to remove redundant code from GP programs during evolution. We then integrated the simplification algorithm into a standard GP system and used it to simplify all programs within certain GP populations. A simplification frequency parameter was used to determine in which generation(s) the GP population would be simplified. This allowed an element of control of how aggressively we would apply simplification.

We used GP systems with our simplification method to perform seven different tasks (three symbolic regression, two image classification and two medical classification) and compared the results to those from a standard GP system.

We found that using simplification led to a reduction in the size of programs held in the GP populations. However, while using simplification at every generation produced the largest reduction in program size in most tasks, it could also lead to an increase in the time used by GP to find a solution. This increase was caused by the overhead that performing simplification adds to the GP system. A simplification frequency of 5 appears to be a good starting point when applying simplification to a new GP task.

We also found that simplification did not have a significant effect on the effectiveness of GP in finding solution programs. This was 
a very important result, as it shows that simplification is a viable technique for combating program bloat problem without sacrificing any of GP's problem solving power.

- We developed two simple building block models, as well as frameworks for analysing these building blocks in order to investigate the effects of simplification on GP populations. We found that simplification both creates and disrupts building blocks within GP populations. These two effects may balance out which keeps GP using simplification as effective as the standard GP.

Four systems were tested, made up of both the standard GP system without simplification and three separate GP systems employing simplification. Each GP system with simplification used a different frequency (every generation, every 5 generations and every 10). Aggregate results on two symbolic regression tasks of different difficulty show that overall, GP systems employing simplification could obtain a slight improvement in fitness at some frequencies, while also reducing program size and shortening evolution times.

Our first building block model was numerical-nodes, which we tracked during individual GP system runs for all four systems, on a heavily constant-dependent symbolic regression task $\left(\right.$ Regression $\left._{4}\right)$. The values of every numerical-node in each generation, as well as a count of distinct numerical-node values in each generation were recorded during the runs.

Through analysis of the data using graphical plots, we found that simplification does disrupt existing building blocks early in the GP evolution. However, simplification also creates new numerical-nodes which were not present in the GP system before. Graphical plots show that some of these new nodes are propagated through the GP populations, constituting new building blocks.

These results provide evidence to help alleviate concerns that using 
a simplification component may hinder building blocks within a GP population and hence the performance of the GP system. Counts of the number of distinct numerical-nodes in each generation show that GP systems with simplification display increased diversity in the numerical-nodes available to the system.

For our second model, we built upon the numerical-node model we used in chapter 5 and used a more complex fixed-depth subtree model. This model consisted of using subtrees of a specified fixed depth.

In order to properly track and visualise these subtrees, we developed FST-Hash, a hashing method to convert these subtrees into numeric values for plotting into graphs for visualisation. FST-Hash is a two step process, the first step consisting of translating the prefix ordering of the standard GP tree program into a level ordering. The second step translated each node into a corresponding enumerated digit (of a particular base, depending on the problem) and converting the resulting numeric sequence into a base-10 (decimal) number for plotting.

We analysed the same Regression 4 problem that we used for the numerical-node model in chapter 5 . Our results confirmed the results from chapter 5 , and the simplification process was found to both disrupt and create new subtrees and building blocks. We found that some highly populated subtrees in the standard GP runs were not found in the runs using GP, indicating that simplification removes important building blocks from GP (although many of these subtrees simply manipulated numerical-nodes). However, we also found that many important building blocks were retained in systems using simplification.

By tracking the number of subtrees being disrupted and created from generation to generation, we found that using simplification at any frequency leads to an increase in both the amount of subtrees being 
disrupted and created in each generation, over the standard GP's "natural" levels. While this higher genetic turnover could lead to higher amounts of genetic diversity, the overall net effect (subtrees created - subtrees disrupted) was relatively consistent between the standard GP and the systems using simplification.

Our findings help explain why using simplification does not appear to have any significant negative effect on the fitness of solution programs found by GP.

- We developed a new method SCHEME, for caching subtrees during fitness evaluation of GP populations. We showed that by using this method, we could significantly reduce both the number of node evaluations required to perform fitness evaluation and the amount of time that GP takes to evolve solutions. Using a caching depth of 2 or 3 is most effective for the six tasks we tested.

Subtree Caching using a Hashing for Equivalence MEthod (SCHEME) utilised a hash based cache and Algebraic Equivalence Hashing to cache fitness evaluations of previously seen subtrees. By using algebraic equivalence hashing, we may avoid evaluating non-previously seen subtrees if they are algebraically equivalent to a previously seen subtree.

We compared the performance of a GP system using SCHEME to the standard GP system using six different tasks of varying difficulty. We found that there is a large reduction in the number of node evaluations that are performed in evaluating the GP programs during the GP runs when using SCHEME. We also found a significant reduction in the CPU time required to perform each GP run, although this reduction varied with each task. Statistical significance testing showed that SCHEME did not significantly affect the training fitnesses and testing accuracies obtained on the six tasks, showing that this is a viable method of reducing the costs of evaluation without deterio- 
rating GP effectiveness.

We found that caching subtrees of depth 2 or 3 was the most effective for each of the six tasks. While four out of the six tasks had the lowest CPU times with a caching depth of 3 , four out of the six tasks had the lowest number of node evaluations when using a caching depth of 2 , suggesting that obtaining the lowest number of node evaluations may not equate to the lowest CPU time (although the times for these two depths are close) and this phenomenon needs further investigation. A caching depth of 2 or 3 may be a good starting point when using this caching approach on other GP tasks.

\subsubsection{Indirect Conclusions}

- We found that using simplification may aid the GP system in particular tasks where the chosen numerical-node range is inappropriate for the given task. As simplification can create new numericalnodes out side of this range, systems which use simplification are less sensitive to this parameter setting. This is particularly useful for problems in which prior knowledge of the parameter ranges is not known.

Additional results using different initial numerical-node ranges (in effect adjusting the difficulty of the task) showed that using simplification resulted in the GP system performing better (and with more stability) than the standard GP in situations where the initial range was highly unsuitable for the problem, suggesting that the creation of new building blocks is highly useful in these situations.

- We modified the fitness evaluation method in GP to avoid re-evaluation of programs born out of reproduction (i.e. elitism). We found that using this simple approach on six tasks, we could gain between $3.5 \%$ and $7 \%$ in the amount of CPU time used by GP to find a solution, when using a "typical" reproduction rate of $10 \%$. 
- We developed two simple method for dynamically selecting caching depth for SCHEME during the evolutionary process. We found that using these methods were not as effective as using an "optimal" static caching depth value. While node evaluations were further reduced, overhead introduced by the dynamic selection led to worse CPU times than using SCHEME with the "optimal" static caching depth. However, using these dynamic methods still achieved significantly better CPU times than the standard GP.

Our first method was to begin with the smallest caching depth and increment the depth at regular intervals during the GP evolution (iSCHEME). The second was to track the cache activity for subtrees and only cache subtrees that contained frequently used subtress (dSCHEME). Both of these methods were used on the same six GP tasks as the original SCHEME and the results of all three compared. It was found that while using these dynamic methods reduces the number of node evaluations and improves cache efficiency, the CPU time required for GP runs using these methods was generally worse than when using the "optimal" cache depth with the original SCHEME. This is most likely caused by the extra overhead introduced by the caching depth selection algorithms.

\subsection{Future Works}

- Dynamic methods of selecting caching depth. We investigated two methods of selecting caching depth during the evolution for SCHEME to use. While these methods further reduced the number of node evaluations, they slightly increased the CPU time that GP evolution took (compared to SCHEME using the "optimal" caching depth). In future, we will investigate more dynamic methods. 
- More complex building block models. The numeric-node and fixeddepth subtree models provided two methods of analysing the dynamics of building blocks within evolved programs in GP. Compared with the existing work in building block analysis in GP, these approaches are certainly a major step. However, these models are far from ideal for building block analysis. We will investigate more effective methods for performing building block analysis in future.

- Adjusting proportion for simplification. While we have looked at adjusting the frequency of how often simplification is performed, our simplification method still simplifies all programs in the GP populations. In future we will look at adjusting the proportion of the population which simplification will be applied to. 


\section{Bibliography}

[1] Albert, J. H. Calculated bets: Computers, gambling, and mathematical modeling to win. The American Statistician 56 (November 2002), 329-330.

[2] Alpaydin, E. Introduction to Machine Learning (Adaptive Computation and Machine Learning). The MIT Press, October 2004.

[3] Altenberg, L. The evolution of evolvability in genetic programming. In Advances in Genetic Programming. MIT Press, 1994, pp. 4774 .

[4] Altenberg, L. The Schema Theorem and Price's Theorem. In Foundations of Genetic Algorithms 3 (Estes Park, Colorado, USA, 31 -2 1994 1995), Morgan Kaufmann, pp. 23-49.

[5] AsHlock, W., AND AsHlOcK, D. Single parent genetic programming. In Proceedings of the 2005 IEEE Congress on Evolutionary Computation (Edinburgh, UK, 2-5 Sept. 2005), D. Corne, Z. Michalewicz, M. Dorigo, G. Eiben, D. Fogel, C. Fonseca, G. Greenwood, T. K. Chen, G. Raidl, A. Zalzala, S. Lucas, B. Paechter, J. Willies, J. J. M. Guervos, E. Eberbach, B. McKay, A. Channon, A. Tiwari, L. G. Volkert, D. Ashlock, and M. Schoenauer, Eds., vol. 2, IEEE Press, pp. 1172-1179. 
[6] AsUnCION, A., AND NEWMAN, D. J. UCI machine learning repository, http://www.ics.uci.edu/ mlearn/MLRepository.html, 2007.

[7] BANZHAF, W. Genetic programming for pedestrians. In Proceedings of the 5th International Conference on Genetic Algorithms, ICGA-93 (University of Illinois at Urbana-Champaign, 17-21 July 1993), S. Forrest, Ed., Morgan Kaufmann, p. 628.

[8] Beyer, H.-G., AND SCHWEFEL, H.-P. Evolution strategies - a comprehensive introduction. Natural Computing: an international journal 1, 1 (May 2002), 3-52.

[9] BISHOP, C. M. Pattern Recognition and Machine Learning (Information Science and Statistics). Springer-Verlag New York, Inc., Secaucus, NJ, USA, 2006.

[10] Blickle, T., AND Thiele, L. Genetic programming and redundancy. In Genetic Algorithms within the Framework of Evolutionary Computation (Workshop at KI-94, Saarbrücken) (1994), Max-PlanckInstitut für Informatik (MPI-I-94-241), pp. 33-38.

[11] Busch, J., Ziegler, J., Aue, C., Ross, A., SAwitzKi, D., AND BANZHAF, W. Automatic generation of control programs for walking robots using genetic programming. In EuroGP '02: Proceedings of the 5th European Conference on Genetic Programming (London, UK, 2002), Springer-Verlag, pp. 258-267.

[12] Cherowitzo, B. Lecture notes, http: //www-math . cudenver. edu/ wcherowi/courses/m5410/exeucalg.html. Visited on 7 January 2006., 2006.

[13] CHITTY, D. M. A data parallel approach to genetic programming using programmable graphics hardware. In GECCO '07: Proceedings of 
the 9th annual conference on Genetic and evolutionary computation (New York, NY, USA, 2007), ACM Press, pp. 1566-1573.

[14] Daida, J. M., Bertram, R. R., Polito, J. A., and Stanhope, $\mathrm{S}$. A. Analysis of single-node (building) blocks in genetic programming. Advances in genetic programming: volume 3 (1999), 217-241.

[15] Daida, J. M., Polito, J. A., Stanhope, S. A., Bertram, R. R., KHOO, J. C., AND CHAUdharY, S. A. What makes a problem GPhard? Analysis of a tunably difficult problem in genetic programming. In Proceedings of the Genetic and Evolutionary Computation Conference (13-17 1999), vol. 2, Morgan Kaufmann, pp. 982-989.

[16] Daida, J. M., SAmples, M. E., AND Byom, M. J. Probing for limits to building block mixing with a tunably-difficult problem for genetic programming. In GECCO '05: Proceedings of the 2005 conference on Genetic and evolutionary computation (New York, NY, USA, 2005), ACM, pp. 1713-1720.

[17] Dorigo, M., And StÜtZle, T. Ant Colony Optimization. Bradford Book, 2004.

[18] EIBEN, A., AND SMITH, J. Introduction to evolutionary computing. Springer, 2003.

[19] EKART, A. Shorter fitness preserving genetic programs. In Articial Evolution. 4th European Conference, AE'99, Selected Papers (2000), vol. 1829, Springer, pp. 73-83.

[20] EKLUnd, S. E. A massively parallel GP engine in VLSI. In Proceedings of the 2002 Congress on Evolutionary Computation CEC2002 (2002), D. B. Fogel, M. A. El-Sharkawi, X. Yao, G. Greenwood, H. Iba, P. Marrow, and M. Shackleton, Eds., IEEE Press, pp. 629-633. 
[21] FIKES, R., AND Nilsson, N. Strips: A new approach to the application of theorem proving to problem solving. Artificial Intelligence 2 (1971), 189-208.

[22] Forrest, S., AND Mitchell, M. Relative building-block fitness and the building-block hypothesis. In Foundations of Genetic Algorithms 2. Morgan Kaufmann, 1993, pp. 109-126.

[23] Gathercole, C., AND Ross, P. Dynamic training subset selection for supervised learning in genetic programming. In Parallel Problem Solving from Nature III (Jerusalem, 9-14 1994), Y. Davidor, H.-P. Schwefel, and R. Männer, Eds., vol. 866, Springer-Verlag, pp. 312321.

[24] Giacobini, M., Tomassini, M., And VAnneschi, L. Limiting the number of fitness cases in genetic programming using statistics. In PPSN VII: Proceedings of the 7th International Conference on Parallel Problem Solving from Nature (London, UK, 2002), Springer-Verlag, pp. 371-380.

[25] GoldberG, D. E. Genetic Algorithms in Search, Optimization and Machine Learning. Addison-Wesley Longman Publishing Co., Inc., Boston, MA, USA, 1989.

[26] Gonnet, G. H. Determining equivalence of expressions in random polynomial time. In STOC '84: Proceedings of the sixteenth annual ACM symposium on Theory of computing (New York, NY, USA, 1984), ACM, pp. 334-341.

[27] GREENIE, D. Converting from base 6 to 10 and back, http: //mathforum.org/library/drmath/view/55738.html (fetched March 2008), 2001.

[28] Gustafson, S., Ekart, A., Burke, E., And Kendall, G. Problem difficulty and code growth in genetic programming. In Genetic Pro- 
gramming and Evolvable Machines 5(3 (2004), Kluwer Academic Publishers, pp. 271-290.

[29] HARDing, S., AND BANZHAF, W. Fast genetic programming on GPUs. In EuroGP (2007), pp. 90-101.

[30] Heitktter, J., and Beasley, D. The hitch-hiker's guide to evolutionary computation: A list of frequently asked questions (FAQ), ftp://rtfm.mit.edu/pub/usenet/news.answers/ ai-faq/genetic/, 2000.

[31] Holland, J. H. Adaptation in natural and artificial systems. In Report of the Systems Analysis Research Group SYS (Ann Arbor, MI: The University of Michigan, 1975), vol. 1/92, The University of Michigan Press.

[32] Holland, J. H. Adaptation in Natural and Artificial Systems: An Introductory Analysis with Applications to Biology, Control and Artificial Intelligence. MIT Press, Cambridge, MA, USA, 1992.

[33] Hooper, D., AND FlanN, N. S. Improving the accuracy and robustness of genetic programming through expression simplification. In Genetic Programming 1996: Proceedings of the First Annual Conference (1996), MIT Press, p. 428.

[34] IBA, H., AND DE GARIS, H. Extending genetic programming with recombinative guidance. Advances in genetic programming: volume 2 (1996), 69-88.

[35] IGel, C., AND Chellapilla, K. Investigating the influence of depth and degree of genotypic change on fitness in genetic programming. In Proceedings of the Genetic and Evolutionary Computation Conference (Orlando, Florida, USA, 1999), W. Banzhaf, J. Daida, A. E. Eiben, M. H. Garzon, V. Honavar, M. Jakiela, and R. E. Smith, Eds., vol. 2, Morgan Kaufmann, pp. 1061-1068. 
[36] JACKSON, D. Fitness evaluation avoidance in boolean GP problems. In Proceedings of the 2005 IEEE Congress on Evolutionary Computation (Edinburgh, UK, 2-5 Sept. 2005), D. Corne, Z. Michalewicz, M. Dorigo, G. Eiben, D. Fogel, C. Fonseca, G. Greenwood, T. K. Chen, G. Raidl, A. Zalzala, S. Lucas, B. Paechter, J. Willies, J. J. M. Guervos, E. Eberbach, B. McKay, A. Channon, A. Tiwari, L. G. Volkert, D. Ashlock, and M. Schoenauer, Eds., vol. 3, IEEE Press, pp. 2530-2536.

[37] KeIJZer, M. Alternatives in subtree caching for genetic programming. In EuroGP (2004), pp. 328-337.

[38] Kennedy, J., And Eberhart, R. C. Swarm intelligence. Morgan Kaufmann Publishers Inc., San Francisco, CA, USA, 2001.

[39] KoHAVI, R. A study of cross-validation and bootstrap for accuracy estimation and model selection. In IJCAI (1995), pp. 1137-1145.

[40] KozA, J. R. Genetic Programming: On the Programming of Computers by Means of Natural Selection (Complex Adaptive Systems). The MIT Press, December 1992.

[41] KozA, J. R. Genetic programming: On the programming of computers by means of natural selection. Statistics and Computing 4, 2 (1994).

[42] Koza, J. R., Keane, M. A., Streeter, M. J., Mydlowec, W., YO, J., AND LANZA, G. Genetic Programming IV : Routine HumanCompetitive Machine Intelligence (Genetic Programming). Springer, 2003.

[43] LANGDON, W. B. Quadratic bloat in genetic programming. In Proceedings of the Genetic and Evolutionary Computation Conference (GECCO-2000) (Las Vegas, Nevada, USA, 10-12 2000), D. Whitley, 
D. Goldberg, E. Cantu-Paz, L. Spector, I. Parmee, and H.-G. Beyer, Eds., Morgan Kaufmann, pp. 451-458.

[44] LANGdON, W. B., AND BANZHAF, W. Repeated patterns in genetic programming. Natural Computing (2008). Published online: 26 May 2007.

[45] Langdon, W. B., AND POli, R. Fitness causes bloat. In Second On-line World Conference on Soft Computing in Engineering Design and Manufacturing (July 1997), P. K. Chawdhry, R. Roy, and R. K. Pan, Eds., Springer-Verlag London, pp. 13-22.

[46] Langdon, W. B., AND POLI, R. Fitness causes bloat: Mutation. In Proceedings of the First European Workshop on Genetic Programming (Paris, JanuaryApril-May 1998), W. Banzhaf, R. Poli, M. Schoenauer, and T. C. Fogarty, Eds., vol. 1391, Springer-Verlag, pp. 37-48.

[47] Langdon, W. B., AND POli, R. Foundations of Genetic Programming. Springer-Verlag, 2002.

[48] LIDL, R., AND NIEDERREITER, H. Introduction to finite fields and their applications. Cambridge University Press, New York, NY, USA, 1986.

[49] LuKE, S. Code growth is not caused by introns. In Late Breaking Papers at the 2000 Genetic and Evolutionary Computation Conference (Las Vegas, Nevada, USA, 8 2000), D. Whitley, Ed., pp. 228-235.

[50] LuKe, S., AND PANAIT, L. Lexicographic parsimony pressure. In Proceedings of the Genetic and Evolutionary Computation Conference (GECCO2002) (2002), Morgan Kaufmann.

[51] MaCKAY, D. J. C. Information Theory, Inference, and Learning Algorithms. Cambridge University Press, 2003. Available from http: //www.inference.phy.cam.ac.uk/mackay/itila/. 
[52] MAJEeD, H. A new approach to evaluate GP schema in context. In Genetic and Evolutionary Computation Conference (GECCO2005) workshop program (2005), ACM Press, pp. 378-381.

[53] MARTIN, W. A. Determining the equivalence of algebraic expressions by hash coding. J. ACM 18, 4 (1971), 549-558.

[54] MCPhee, N. F., AND Miller, J. D. Accurate replication in genetic programming. In Genetic Algorithms: Proceedings of the Sixth International Conference (ICGA95) (Pittsburgh, PA, USA, 15-19 1995), L. Eshelman, Ed., Morgan Kaufmann, pp. 303-309.

[55] Mitchell, M. An Introduction to Genetic Algorithms (Complex Adaptive Systems). The MIT Press, February 1998.

[56] MitchelL, T. Machine Learning. McGraw Hill, 1997.

[57] NoRDIN, P. A compiling genetic programming system that directly manipulates the machine code. In Advances in Genetic Programming, K. E. Kinnear, Jr., Ed. MIT Press, 1994, ch. 14, pp. 311-331.

[58] Nordin, P., AND BANZHAF, W. Complexity compression and evolution. In Genetic Algorithms: Proceedings of the Sixth International Conference (ICGA95) (Pittsburgh, PA, USA, 15-19 1995), L. Eshelman, Ed., Morgan Kaufmann, pp. 310-317.

[59] Nordin, P., Francone, F., And BAnZhaf, W. Explicitly defined introns and destructive crossover in genetic programming. In Proceedings of the Workshop on Genetic Programming: From Theory to RealWorld Applications (9 July 1995), pp. 6-22.

[60] O'ReILly, U.-M. An analysis of genetic programming. PhD thesis, Carleton University, Ottawa, Canada, 1995. Adviser-Franz Oppacher.

[61] O'ReILly, U.-M., AND OPPACHER, F. The troubling aspects of a building block hypothesis for genetic programming. In FOGA 
(1994), L. D. Whitley and M. D. Vose, Eds., Morgan Kaufmann, pp. 73-88.

[62] PERKIS, T. Stack-based genetic programming. In Proceedings of the 1994 IEEE World Congress on Computational Intelligence (Orlando, Florida, USA, 27-29 June 1994), vol. 1, IEEE Press, pp. 148-153.

[63] Peterson, W., AND BROWN, D. Cyclic codes for error detection. In Proceedings of the IRE (1961), vol. 49, pp. 228-235.

[64] PIszcz, A., AND Soule, T. Dynamics of evolutionary robustness. In GECCO '06: Proceedings of the 8th annual conference on Genetic and evolutionary computation (New York, NY, USA, 2006), ACM, pp. 871878.

[65] POLI, R. Discovery of symbolic, neuro-symbolic and neural networks with parallel distributed genetic programming. Tech. Rep. CSRP-96-14, University of Birmingham, School of Computer Science, Aug. 1996. Presented at 3rd International Conference on Artificial Neural Networks and Genetic Algorithms, ICANNGA'97.

[66] POLI, R. Genetic programming for image analysis. In Genetic Programming 1996: Proceedings of the First Annual Conference (Stanford University, CA, USA, 28-31 July 1996), J. R. Koza, D. E. Goldberg, D. B. Fogel, and R. L. Riolo, Eds., MIT Press, pp. 363-368.

[67] POLI, R. Exact schema theorem and effective fitness for GP with one-point crossover. In Proceedings of the Genetic and Evolutionary Computation Conference (GECCO-2000) (Las Vegas, Nevada, USA, 10-12 2000), D. Whitley, D. Goldberg, E. Cantu-Paz, L. Spector, I. Parmee, and H.-G. Beyer, Eds., Morgan Kaufmann, pp. 469-476.

[68] POLI, R. A macroscopic exact schema theorem and a redefinition of effective fitness for GP with one-point crossover. Tech. Rep. CSRP00-1, University of Birmingham, 2000. 
[69] POLI, R. Exact schema theory for genetic programming and variable-length genetic algorithms with one-point crossover. Genetic Programming and Evolvable Machines 2, 2 (2001), 123-163.

[70] POLI, R., AND LANGDON, W. B. An experimental analysis of schema creation, propagation and disruption in genetic programming. In Genetic Algorithms: Proceedings of the Seventh International Conference (Michigan State University, East Lansing, MI, USA, 19-23 1997), T. Back, Ed., Morgan Kaufmann, pp. 18-25.

[71] POli, R., AND LAngdon, W. B. A new schema theory for genetic programming with one-point crossover and point mutation. In Genetic Programming 1997: Proceedings of the Second Annual Conference (Stanford University, CA, USA, 13-16 1997), J. R. Koza, K. Deb, M. Dorigo, D. B. Fogel, M. Garzon, H. Iba, and R. L. Riolo, Eds., Morgan Kaufmann, pp. 278-285.

[72] Poli, R., Langdon, W. B., And McPhee, N. F. A Field Guide to Genetic Programing. Published via http://lulu.com and freely available at http: //www.gp-field-guide.org •uk, 2008.

[73] POli, R., AND McPheE, N. F. Exact GP schema theory for headless chicken crossover and subtree mutation. In Proceedings of the 2001 Congress on Evolutionary Computation CEC2001 (COEX, World Trade Center, 159 Samseong-dong, Gangnam-gu, Seoul, Korea, 2730 2001), IEEE Press, pp. 1062-1069.

[74] Poli, R., AND MCPHEE, N. F. Exact schema theorems for GP with one-point and standard crossover operating on linear structures and their application to the study of the evolution of size. In Genetic Programming, Proceedings of EuroGP'2001 (Lake Como, Italy, 18-20 2001), J. F. Miller, M. Tomassini, P. L. Lanzi, C. Ryan, A. G. B. Tettamanzi, and W. B. Langdon, Eds., vol. 2038, Springer-Verlag, pp. 126-142. 
[75] Poli, R., And McPheE, N. F. Exact schema theory for GP and variable-length GAs with homologous crossover. In Proceedings of the Genetic and Evolutionary Computation Conference (GECCO-2001) (San Francisco, California, USA, 7-11 2001), L. Spector, E. D. Goodman, A. Wu, W. B. Langdon, H.-M. Voigt, M. Gen, S. Sen, M. Dorigo, S. Pezeshk, M. H. Garzon, and E. Burke, Eds., Morgan Kaufmann, pp. 104-111.

[76] Poli, R., AND McPheE, N. F. General schema theory for genetic programming with subtree-swapping crossover: Part II. Evol. Comput. 11, 2 (2003), 169-206.

[77] ROBERTS, M. The effectiveness of cost based subtree caching mechanisms in typed genetic programming for image segmentation. In Applications of Evolutionary Computation, Proceedings of EvoIASP 2003 (Essex, UK, 2003), vol. 2611, Springer-Verlag.

[78] RoscA, J. P. Analysis of complexity drift in genetic programming. In Genetic Programming 1997: Proceedings of the Second Annual Conference (Stanford University, CA, USA, 13-16 1997), J. R. Koza, K. Deb, M. Dorigo, D. B. Fogel, M. Garzon, H. Iba, and R. L. Riolo, Eds., Morgan Kaufmann, pp. 286-294.

[79] Rosca, J. P., AND BAllaRD, D. H. Rooted-tree schemata in genetic programming. In Advances in Genetic Programming 3. MIT Press, 1999, ch. 11, pp. 243-271.

[80] Ryan, C., Majeed, H., AND Azad, A. A competitive building block hypothesis. In Genetic and Evolutionary Computation GECCO 2004 (2004), vol. 3103/2004, Springer Berlin / Heidelberg, pp. 654665. 
[81] Sastry, K., O’Reilly, U., GOldberG, D., ANd Hill, D. Building block supply in genetic programming. Genetic Programming Theory and Practice.

[82] Silva, S., AND CostA, E. Comparing tree depth limits and resource-limited GP. Evolutionary Computation, 2005. The 2005 IEEE Congress on 1 (Sept. 2005), 920-927 Vol.1.

[83] SMART, W. Victoria Genetic Programming (VGP) package, Available from http://homepages.mcs.vuw.ac.nz/ mengjie/. Last visited on 2 October 2008., 2006.

[84] Smart, W., Andreae, P., AND ZhanG, M. Empirical analysis of GP tree-fragments. In Proceedings of the 10th European Conference on Genetic Programming (Valencia, Spain, 11 - 13 2007), M. Ebner, M. O’Neill, A. Ekárt, L. Vanneschi, and A. I. Esparcia-Alcázar, Eds., vol. 4445 of Lecture Notes in Computer Science, Springer, pp. 55-67.

[85] SMITH, III, J. F. Genetic program based data mining for fuzzy decision trees. In IDEAL (2004), Z. R. Yang, R. M. Everson, and H. Yin, Eds., vol. 3177 of Lecture Notes in Computer Science, Springer, pp. 464470.

[86] SMITH, III, J. F. Evolving fuzzy decision tree structure that adapts in real-time. In GECCO '05: Proceedings of the 2005 conference on Genetic and evolutionary computation (New York, NY, USA, 2005), ACM, pp. 1737-1744.

[87] SOUle, T., AND Foster, J. A. Effects of code growth and parsimony pressure on populations in genetic programming. Evolutionary Computation 6, 4 (Winter 1998), 293-309.

[88] Soule, T., Foster, J. A., AND Dickinson, J. Code growth in genetic programming. In Genetic Programming 1996: Proceedings of the First Annual Conference (28-31 1996), MIT Press, pp. 215-223. 
[89] Soule, T., And Heckendorn, R. B. An analysis of the causes of code growth in genetic programming. Genetic Programming and Evolvable Machines 3, 3 (2002), 283-309.

[90] STREETER, M. J. The root causes of code growth in genetic programming. In Proceedings of Genetic Programming: 6th European Conference, EuroGP 2003, Essex, UK, April 14-16, 2003. (2003), Springer-Verlag, pp. $443-454$.

[91] TetKo, I. V., Livingstone, D. J., And Luik, A. I. Neural network studies, 1 . comparison of overfitting and overtraining. Journal of Chemical Information and Computer Sciences 35, 5 (1995), 826-833.

[92] Trappe, W., AND Washington., L. C. Introduction to Cryptography and Coding Theory. Prentice-Hall, 2nd edition, 2006.

[93] Whigham, P. A. Grammatically-based genetic programming. In Proceedings of the Workshop on Genetic Programming: From Theory to Real-World Applications (Tahoe City, California, USA, 9 1995), J. P. Rosca, Ed., pp. 33-41.

[94] Whigham, P. A. A schema theorem for context-free grammars. In 1995 IEEE Conference on Evolutionary Computation (Perth, Australia, 29 - 1 1995), vol. 1, IEEE Press, pp. 178-181.

[95] Wilson, G. C., AND Heywood, M. I. Context-based repeated sequences in linear genetic programming. In EuroGP (2005), pp. 240249 .

[96] WONG, M. L., AND LEUNG, K. S. Evolutionary program induction directed by logic grammars. Evolutionary Computation 5, 2 (summer 1997), 143-180.

[97] Xie, H., Zhang, M., And Andreae, P. Population clustering in genetic programming. In Proceedings of the 9th European Conference 
on Genetic Programming (Budapest, Hungary, 10 - 12 2006), P. Collet, M. Tomassini, M. Ebner, S. Gustafson, and A. Ekárt, Eds., vol. 3905 of Lecture Notes in Computer Science, Springer, pp. 190-201.

[98] ZHANG, B.-T., AND CHO, D.-Y. Genetic programming with active data selection. Lecture Notes in Computer Science 1585 (1999), 146-153.

[99] ZHANG, B.-T., AND MÜHLENBEIN, H. Balancing accuracy and parsimony in genetic programming. Evolutionary Computation 3, 1 (1995), 17-38.

[100] ZHANG, M., AND CIESIELSKI, V. Genetic programming for multiple class object detection. In 12th Australian Joint Conference on Artificial Intelligence (Sydney, Australia, 6-10 Dec. 1999), N. Foo, Ed., vol. 1747 of LNAI, Springer-Verlag, pp. 180-192.

[101] Zhang, M., ZHANG, Y., AND SMART, W. D. Program simplification in genetic programming for object classification. In Proceedings of Knowledge-based intelligent information and engineering systems: (Part I,III-IV) : 9th international conference (2005), pp. 988-996.

[102] ZHANG, Y. Genetic programming for multiple class classification, Honours Report, 2004.

[103] ZHONG, X., AND SOUlE, T. Growth of self-canceling code in evolutionary systems. In GECCO '06: Proceedings of the 8th annual conference on Genetic and evolutionary computation (New York, NY, USA, 2006), ACM, pp. 223-228. 